\title{
Electronic Structure of the Rare-Earth Nitrides
}

by

A. R. H. Preston

A thesis

submitted to the Victoria University of Wellington

in fulfilment of the

requirements for the degree of

Doctor of Philosophy

in Physics.

Victoria University of Wellington

2010 



\begin{abstract}
The rare-earth nitrides (ReNs) are a class of novel materials with potential for use in spintronics applications. Theoretical studies indicate that among the ReNs there could be half-metals, semimetals and semiconductors, all exhibiting strong magnetic ordering. This is because of the complex interaction between the partially filled rare-earth $4 f$ orbital and the nitrogen $2 p$ valence and rare-earth $5 d$ conduction bands.

This thesis uses experimental and theoretical techniques to probe the ReN electronic structure. Thin films of SmN, EuN, GdN, DyN, LuN and HfN have been produced for study. Basic characterization shows that the films are of a high quality. The result of electrical transport, magnetometry, and optical and x-ray spectroscopy are interpreted to provide information on the electronic structure. SmN, GdN, DyN are found to be semiconductors in their ferromagnetic ground state while HfN is a metal. Results are compared with density functional theory (DFT) based calculations. The free parameters resulting from use of the local spin density approximation with Hubbard- $U$ corrections as the exchange-correlation functional are adjusted to reach good agreement with x-ray absorption and emission spectroscopy at the nitrogen $\mathrm{K}$-edge. Resonant $\mathrm{x}$-ray emission is used to experimentally measure valence band dispersion of GdN. No evidence of the rare-earth $4 f$ levels is found in any of the K-edge spectroscopy, which is consistent with the result of M-edge x-ray absorption which show that the $4 f$ wave function of the rare-earths in the ReNs are very similar to those of rare-earth metal.

An auxillary resonant x-ray emission study of $\mathrm{ZnO}$ is used to map the dispersion of the electronic band structure across a wide range of the Brillouin zone. The data, and calculations based on $G W$ corrections to DFT, together provide a detailed picture of the bulk electronic band structure.
\end{abstract}





\section{Acknowledgments}

I must acknowledge the assistance, encouragement and opportunities provided to me by my supervisors, Ben Ruck and Joe Trodahl. I could not have asked for better supervisors. I greatly appreciate the start that Ben gave me in physics and will miss the many discussions from which I learned so much. Joe did an excellent job of keeping me on track - now can we go sailing?

I must also acknowledge the other members of our research group: Felix Budde, Annette Koo, Simon Granville, Claire Meyer, Bart Ludbrook and Jan Richter. And those I have collaboratored with: Louis Piper, Kevin Smith and Alex DeMasi at BU; James Downes and Josh Brown at MacQuarie; Ian Farrell at Canterbury; and Walter Lambrecht at CWRU. In particular I would like to recognize Bart for his assistance with film growth, Louis and James for sharing their knowledge of synchrotron experiments, and Walter for his help with theory and for his hospitality, which included my first Thanksgiving.

Daniel Johnston helped greatly with the editing of my thesis. He didn't speak physics before, but he does now. I very much appreciate the help and hope to return the favour one day.

Friends made my time in Wellington a pleasure, especially those in the Raman lab: Pablo Etchegoin, who was almost like a third supervisor to me, Chris, Matthias, Evan, Eric, and Shrividia.

I would like to recognize Hollie, my parents, and my siblings for being so wonderfully supportive through it all. Finally, I would like to acknowledge my grandfather, Tony Bray, who provided me with so much inspiration.

To all of you, thanks. 



\section{Contents}

1 Introduction $\quad 1$

1.1 Outline ........................ . . . 4

1.2 Attribution ................... 5

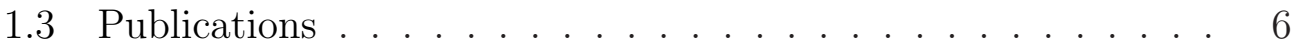

2 The Rare-Earth Nitrides $\quad 9$

2.1 Rare-Earths . . . . . . . . . . . . . . . . . . . . 9 9

2.2 Nitrides . . . . . . . . . . . . . . . . . . . . . . 9

2.3 Rare-Earth Nitrides . . . . . . . . . . . . . . . . . . . . 10

2.4 Hafnium Nitride . . . . . . . . . . . . . . . . . . . . . . . 12

3 Theoretical Background 13

3.1 The Hartree-Fock Approximation . . . . . . . . . . . . . . . . 14

3.2 Density Functional Theory . . . . . . . . . . . . . . . 16

3.3 Atomic Multiplets . . . . . . . . . . . . . . . . . . . . 19

3.4 Electronic Band Structure . . . . . . . . . . . . . . . 26

4 Experimental Band Structure Probes 31

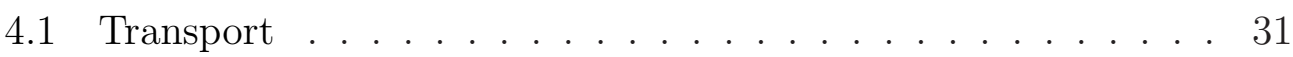

4.2 Magnetometry . . . . . . . . . . . . . . . . . . 34

4.3 Optical Transmission . . . . . . . . . . . . . . 36

4.4 X-ray Spectroscopy . . . . . . . . . . . . . . . . 37 
5 Growth and Basic Characterization 45

5.1 Film Deposition Techniques . . . . . . . . . . . . . . . 46

5.2 Structural Characterization . . . . . . . . . . . . 51

5.3 Considerations for X-ray Spectroscopy . . . . . . . . . . . 58

5.4 Conclusion . . . . . . . . . . . . . . . . . . . 60

$\begin{array}{lll}6 & \mathrm{GdN} & 61\end{array}$

6.1 Motivation . . . . . . . . . . . . . . . . 61

6.2 Transport . . . . . . . . . . . . . . . . . 63

6.3 Magnetism . . . . . . . . . . . . . . . . . 65

6.4 Transport and Magnetism . . . . . . . . . . . . . . 67

6.5 Optical Transmission . . . . . . . . . . . . . . . . . . 69

6.6 Band Structure . . . . . . . . . . . . . . . . 71

6.7 X-ray Spectroscopy . . . . . . . . . . . . . . . . . . . . 74

6.8 Conclusion and Outlook . . . . . . . . . . . . . . . . . 82

7 SmN and DyN $\quad 85$

7.1 Motivation . . . . . . . . . . . . . . . . . . . 85

7.2 Transport and Magnetism . . . . . . . . . . . . . 86

7.3 Electronic Band Structure . . . . . . . . . . . . . . . . . . 92

7.4 Optical Spectroscopy . . . . . . . . . . . . . . . 95

7.5 X-ray Spectroscopy . . . . . . . . . . . . . . . . . 96

7.6 Conclusions and Outlook . . . . . . . . . . . . . . . . . . 98

8 X-ray Spectroscopy Across the ReN Series 101

8.1 Nitrogen K-edge . . . . . . . . . . . . . . . . . . . . . . . . . . 101

8.2 Rare-earth M-edge . . . . . . . . . . . . . . . . . 105

8.3 Conclusion . . . . . . . . . . . . . . . . . . . . . 108

9 Band Structure of $\mathrm{ZnO} \quad 111$

9.1 Motivation . . . . . . . . . . . . . . . . . . . 111

9.2 Experimental Details . . . . . . . . . . . . . . . . . . 113

9.3 Theoretical Details . . . . . . . . . . . . . . . . . . . 114 
9.4 Results . . . . . . . . . . . . . . . . . . . . . 114

9.5 Conclusion . . . . . . . . . . . . . . . . . . . 124

10 Conclusion and Outlook 125

10.1 Results . . . . . . . . . . . . . . . . . 125

10.2 Outlook . . . . . . . . . . . . . . . 127 



\section{List of Figures}

5.1 XRD of GdN, comparison of capped and uncapped samples . . 50

5.2 XRD of the ReNs . . . . . . . . . . . . . . . . 53

$5.3 \mathrm{XRD}$ of GdN, comparison of TED and PLD samples . . . . . 56

5.4 RHEED and microscope images of GdN grown by PLD . . . . 57

5.5 SmN XES at the N K-edge, capping layer effects . . . . . . . . 59

$6.1 \mathrm{GdN} R(\mathrm{~T})$, comparison of samples . . . . . . . . . . . 63

6.2 GdN $\mathrm{M}(\mathrm{T})$ and $\mathrm{M}(\mathrm{H})$, comparison of TED and PLD samples . 66

6.3 GdN R(T) and $\mathrm{M}(\mathrm{T})$, plotted on common axes . . . . . . . 68

6.4 GdN optical transmission . . . . . . . . . . . . . . . . 69

$6.5 \mathrm{GdN}$ band structure . . . . . . . . . . . . . . 72

6.6 GdN XES and XAS at the N K-edge . . . . . . . . . . 75

6.7 GdN RXES at the N K-edge . . . . . . . . . . . . . 77

$6.8 \mathrm{GdN}$ band structure, showing $p$-character . . . . . . . . . . . 80

6.9 GdN XAS at the Gd M-edge . . . . . . . . . . . . . . 81

6.10 GdN PDOS, orbitally resolved . . . . . . . . . . . 83

$7.1 \mathrm{SmN}$ and DyN, R(T) and $\mathrm{M}(\mathrm{T}) \ldots \ldots . \ldots . . \ldots 87$

7.2 $\mathrm{SmN} \mathrm{M}(\mathrm{H}), \mathrm{FC}$ and ZFC at different temperatures . . . . . . 90

$7.3 \mathrm{SmN}$ band structure . . . . . . . . . . . . . . . . . . 92

7.4 DyN band structure . . . . . . . . . . . . . . . 93

7.5 SmN and DyN optical transmission . . . . . . . . . . . 95

7.6 SmN XES and XAS at the N K-edge . . . . . . . . . . . 97 
7.7 DyN XES and XAS at the N K-edge . . . . . . . . . . . . 98

8.1 ReN XES at the N K-edge . . . . . . . . . . . . . . . . . 102

8.2 ReN XAS at the N K-edge . . . . . . . . . . . . . . . . 103

8.3 HfN XES and XAS at the N K-edge . . . . . . . . . . . . . 104

$8.4 \mathrm{Sm}$ and SmN XAS at the Sm M-edge . . . . . . . . . . 105

8.5 EuN XAS at the Eu M-edge . . . . . . . . . . . . . . . . 107

9.1 ZnO XES and XAS at the O K-edge . . . . . . . . . 115

9.2 Comparison between $G W$ and HDFT calculations . . . . . . . 117

$9.3 \mathrm{ZnO}$ band structure, orbitally resolved . . . . . . . . . . . 118

9.4 ZnO CRXES at the O K-edge . . . . . . . . . . . . . . 120

$9.5 \mathrm{ZnO} p_{x y}$ RXES plotted with band structure . . . . . . . . 121

$9.6 \mathrm{ZnO} p_{z}$ RXES plotted with band structure . . . . . . . . . . . 122

9.7 ZnO RXES at the O K-edge, image map . . . . . . . . . . . 123 


\section{List of Tables}

3.1 Calculated atomic parameters for $\mathrm{Gd}^{3+} \ldots$. . . . . . . . . . 21

3.2 Properties of the $\mathrm{Re}^{3+}$ multiplets . . . . . . . . . . . . . 24

4.1 Nomenclature in x-ray spectroscopy . . . . . . . . . . . . . . 39

4.2 Experimental details of XXS reported in this thesis . . . . . . 44

5.1 Lattice constants and crystallite sizes of the ReNs . . . . . . 54 



\title{
Glossary
}

\author{
ARPES Angle resolved photoelectron spectroscopy \\ BIS Bremsstrahlung isochromat spectroscopy \\ CB Conduction band \\ CBM Conduction band minimum \\ CRXES The coherent fraction extracted from RXES; also used to as \\ coherent resonant x-ray emission spectra/spectrum \\ DFT Density functional theory \\ DMS Dilute magnetic semiconductor (e.g., GaMnN) \\ DOS Density of states \\ EXAFS Extended x-ray absorption fine structure \\ FC Field-cooled \\ FTIR Fourier transform infrared spectroscopy \\ GW A technique for approximating the quasiparticle self-energy. \\ $\mathrm{G}$ is the Green's function; W is the screened Coulomb poten- \\ tial \\ HA Hartree approximation \\ HFA Hartree-Fock approximation \\ HSE03 Exchange and correlation functional published by Heyd, Scuse- \\ ria, and Enzerhof in 2003 \\ LMTO Linear muffin tin orbitals
}


L(S)DA Local (spin) density approximation

LSDA $+\mathbf{U} \quad$ Local spin density approximation with Hubbard- $U$ corrections NEXAFS see XAS

NRA Nuclear reaction analysis

Pnictide Row $\mathrm{V}$ of the periodic table (same valence as nitrogen)

PDOS Partial density of states

PFY Partial flourescent yield

PLD Pulsed laser deposition

RBS Rutherford backscattering spectroscopy

Re Rare-earth

ReN Rare-earth nitride

RHEED Reflection high energy electron diffraction

RXES Resonant x-ray emission spectroscopy/spectra/spectrum

Spintronics Spin-electronics; the use of electron spin as well as charge in devices

TED Thermal evaporation deposition

TEY Total electron yield

TFY Total flourescent yield

UHV Ultra high vacuum

VB Valence band

VBM Valence band maximum

XANES See XAS

XAS X-ray absorption spectroscopy/spectra/spectrum (also: NEXAFS, XANES)

XES X-ray emission spectroscopy/spectra/spectrum

XMCD X-ray magnetic circular dichroism 
XRD X-ray diffraction

XPS X-ray photoemission spectroscopy/spectra/spectrum

XXS A catchall phrase for all of XAS, XES and RXES

YSZ Yittria stabilized zirconia

ZFC Zero-field-cooled 



\section{Chapter 1}

\section{Introduction}

The fundamental laws necessary for the mathematical treatment of a large part of physics and the whole of chemistry are thus completely known, and the difficulty lies only in the fact that application of these laws leads to equations that are too complex to be solved.

Paul Dirac, 1930

More than seventy years after the quantum mechanics needed to explain the behaviour of electrons in condensed materials was first discovered the field is still vibrant and interesting. As Dirac observed, it is unlikely that research in this area will ever be complete since the many body problem all but ensures that a complete description of the electrons in any non-trivial system will never be practically possible.

Nevertheless, progress since that observation has been remarkable. Driven primarily by the large scale adoption of integrated circuits and the quest for semiconductors with more desirable properties than silicon, physicists are now able to create extremely high quality single crystals of many materials. Likewise, numerical approximations of the ground state many-electron wave function have improved dramatically. Led by the discovery of the density 
functional theory (DFT) and rapid increases in computing power, improved by the experimental developments referred to above, computational ability has reached the point where it can be claimed that any crystalline material in which the electrons can be considered reasonably independent of each other is well understood [1].

As a result recent interest has moved to strongly correlated materials, systems in which electron-electron interactions can no longer be ignored. This shift in focus depended on the convergence of a number of independent factors. Experimentally, the growth of novel materials is an interesting exercise. Theoretically, strongly correlated materials, which require a relaxation of the independent electron approximation, are perhaps the current "final frontier" in crystalline condensed matter physics. Superconductors and magnetic crystals are both strongly correlated materials with significant economic potential. The latter are used in the rapidly developing field of spintronics (spin-electronics) in which the electron spin and charge are both utilized $[2,3]$.

Perhaps the most impressive spintronic device so far is the magnetic read head found in all modern hard disk drives. Today, the read head is based on tunneling magnetoresistance which allows the detection of significantly smaller magnetic fields than would otherwise be possible. This technology follows the discovery of giant magnetoresistance which spurred the huge increases in magnetic storage density of the last decade, and the 2007 Nobel Prize in Physics [4]. This, however, is just the tip of the iceberg. Many believe it is simply a matter of time before newly available non-volatile magnetic random access memories with the dual advantages of rapid access times and low power consumption come to dominate the market [5]. Other potential applications include spin-field effect transistors, spin-logic devices, novel polarized photon sources, or even quantum computers [2].

The spintronic devices that have been mass produced to date are based on typical magnetic materials like iron or cobalt combined with magnesium oxide, but more advanced applications will require novel materials that show 
the strong spin-charge coupling necessary to effectively manipulate electron spins. In particular magnetic semiconductors and half-metals would be extremely useful. The most studied of these are dilute magnetic semiconductors (DMS), semiconductors that have been doped with a magnetic material. Examples include GaMnAs, GaMnN [6], GaGdN [7], ZnGdO [8], and transition metal doped $\mathrm{ZnO}$ [9], all doped with a few percent of the magnetic ion. However, these materials are plagued by the problem of consistent doping. The magnetic exchange interaction between the magnetic dopants depends heavily on the distance between the individual atoms which is hard to precisely control and simulate. Further, the magnetic elements tend to heavily dope the semiconductor preventing independent control of the carrier concentration, and precipitates and other inhomogeneities are problematic. Unfortunately this restricts potential applications and their main proposed use is as spin injection layers for conventional semiconductors [8].

Any materials that are intrinsically both semiconducting and magnetic are clearly very interesting. In principle it should be possible to control the carrier concentration of such a material without affecting the magnetism. At present only a few intrinsic ferromagnetic semiconductors are known. $\mathrm{CrBr}_{3}$, $\mathrm{EuO}, \mathrm{CuCr}_{2} \mathrm{Te}_{3} \mathrm{I}, \mathrm{DyF}_{3}$ and $\mathrm{EuLiH}_{3}$ have all been studied [10]. The parent compounds of the manganite perovskites that show colossal magnetoresistance are semiconducting, but they are antiferromagnetic, only displaying ferromagnetism and the CMR effect upon doping to degeneracy [11].

Another class of intrinsic magnetic semiconductors are the rare-earth nitrides (ReNs). Modern band structure calculations suggesting that the series contains both half-metals and semiconductors appeared at roughly the same time as the increased interest in novel spintronic materials. Rare-earth elements are defined by their partially filled $4 f$ shell, which increases in occupancy across the series. When bonding with nitrogen the rare-earth atoms each contribute three valence electrons, and the electrons remaining in the unfilled $4 f$ shell are relatively atomic like in nature. This means that the ReN series tends to exhibit very consistent chemical properties, but widely 
varying magnetic behaviour, providing a number of candidates for use as a base or a dopant in spintronic devices [12].

Despite promising early results the theoretical frameworks, which must treat both the delocalized band electrons and the highly-localized $4 f$ shell, and also the interaction between the two, remain in their infancy [13]. Many outstanding questions remain regarding the electronic and magnetic structure of the ReNs, and theoretical progress will be heavily dependent on experimental results. This thesis aims to provide some of the experimental detail necessary in order to gain insight into the electronic structure of many of the ReNs.

\subsection{Outline}

This thesis is comprised of ten chapters, this introduction being the first. The next three chapters provide the necessary background information. Chapter 2 provides an overview of the ReNs, and a brief literature review. Chapter 3 , details the theoretical concepts that underpin the work in this thesis. Chapter 4 outlines the experimental techniques used to probe samples and to elucidate information about their electronic and magnetic structure.

The subsequent four chapters present the results of the studies of the ReNs. Chapter 5 details the growth techniques and basic characterization used to produce the high quality samples needed for electronic investigations. Chapter 6 presents a rather complete theoretical and experimental investigation of the GdN electronic structure. Chapter 7 presents results obtained for $\mathrm{SmN}$ and DyN, in a manner similar to the GdN chapter. Chapter 8 compares all of the x-ray spectroscopy results obtained across the ReN series. $\mathrm{LuN}$ and $\mathrm{HfN}$ are included in the comparison. Results obtained from x-ray spectroscopy at the rare-earth M-edge are also detailed.

The last results chapter, Chapter 9, presents an independent resonant $\mathrm{x}$-ray emission spectroscopy study of the $\mathrm{ZnO}$ band structure which was performed in parallel with the ReN work presented in the rest of the thesis. 
Finally, Chapter 10 concludes the thesis and provides some thoughts on possible future directions for the research.

\subsection{Attribution}

A large experimental project, such as the one my thesis is a part of, relies on the contributions of many people, and collaborations between many groups. I am only a small cog in the wheel and it is important to correctly attribute those who provided assistance and results that appear in this thesis.

- Film growth - Initially by F. Budde, A. Koo, D. Pringle, then by Z. Jhong, and later by J. Richter. Although not a focus of my thesis, I was involved heavily in this work.

- Transport measurements - Involved the entire research group.

- Magnetic measurements - Although this thesis does not focus on the magnetic properites of the ReNs, the electronic ground state of the ReNs is intertwined with any magnetic ordering. I relied heavily on measurements and analysis of magnetism performed initially by S. Granville and later by B. Ludbrook and C. Meyer.

- Optical spectroscopy - Performed with H. J. Trodahl and assistance from A. Bittar, N. Strickland.

- X-ray spectroscopy - A collaboration with K. Smith at the Novel Materials Laboratory, Boston University and J. E. Downes at MacQuarie Univeristy. I was primarily responsible for the measurements, along with B. J. Ruck, J. E. Downes, L. F. J. Piper, K. E. Smith, A. DeMasi and J. Brown.

- Band structure calculations - A collaboration with W. R. L. Lambrecht at Case Western Reserve University.

- Multiplet calculations - Performed by myself. 


\subsection{Publications}

I am a co-author on ten peer-review papers published during the course of this thesis. One other paper has been submitted to Applied Physics Letters.

- Electrical and optical properties of hafnium nitride thin films

I. L. Farrell, R. J. Reeves, S. M. Durbin, A. R. H. Preston, and B. J. Ruck

Appl. Phys. Lett. submitted (2009)

- Electronic band structure information of GdN extracted from x-ray absorption and emission spectroscopy

A. R. H. Preston, H. J. Trodahl, B. J. Ruck, L. F. J. Piper, K. E. Smith, J. E. Downes and W. R. L. Lambrecht

Appl. Phys. Lett. 96, 032101 (2010)

- Magnetic properties of ErN films

C. Meyer, B. J. Ruck, A. R. H. Preston, S. Granville, G. V. M. Williams and H. J. Trodahl

J. Mag. Mag. Mater. in press (2010)

- Growth and properties of epitaxial GdN

B. M. Ludbrook, I. L. Farrell, M. Kuebel, B. J. Ruck, A. R. H. Preston, H. J. Trodahl, S. M. Durbin, R. J. Reeves, L. Ranno, and C. Meyer

J. Appl. Phys. 106, O63910 (2009)

- Vibrational properties of the rare-earth nitrides: Raman spectra and theory

S. Granville, C. Meyer, A. R. H. Preston, B. M. Ludbrook, B. J. Ruck, H. J. Trodahl, T. R. Paudel and W. R. L. Lambrecht

Phys. Rev. B 79, 054301 (2009)

- Near-zero-moment ferromagnetism in the semiconductor SmN

C. Meyer, B. J. Ruck, J. Zhong, S. Granville, A. R. H. Preston, 
G. V. M. Williams, and H. J. Trodahl

Phys. Rev. B 78, 174406 (2008)

- Band structure of ZnO from resonant x-ray emission spectroscopy

A. R. H. Preston, B. J. Ruck, L. F. J. Piper, A. DeMasi, K. E. Smith, A. Schleife, F. Fuchs, F. Bechstedt, J. Chai and S. M. Durbin Phys. Rev. B 78, 155114 (2008)

- Electronic properties of $(G a, M n) N$ thin films with high Mn content S. Granville, B. J. Ruck, A. R. H. Preston, T. Stewart, F. Budde, H. J. Trodahl, A. Bittar, J. E. Downes and M. Ridgway J. Appl. Phys. 104, 103710 (2008)

- Comparison between experiment and calculated band structures for DyN and $S m N$

A. R. H. Preston, S. Granville, D. H. Housden, B. M. Ludbrook, B. J. Ruck, H. J. Trodahl, A. Bittar, G. V. M. Williams, J. E. Downes, A. DeMasi, Y. Zhang, K. E. Smith and W. R. L. Lambrecht Phys. Rev. B 76, 245120 (2008)

- Ferromagnetic redshift of the optical gap in GdN H. J. Trodahl, A. R. H. Preston, J. Zhong, B. J. Ruck, N. M. Strickland, C. Mitra and W. R. L. Lambrecht Phys. Rev. B 76, 085211 (2007)

- Semiconducting ground state of GdN thin films

S. Granville, B. J. Ruck, F. Budde, A. Koo, D. J. Pringle, F. Kuchler, A. R. H. Preston, D. H. Housden, N. Lund, A. Bittar, G. V. M. Williams and H. J. Trodahl

Phys. Rev. B 73, 235335 (2008) 



\section{Chapter 2}

\section{The Rare-Earth Nitrides}

\section{$2.1 \quad$ Rare-Earths}

Rare-earth elements have, by definition, a partially filled $4 f$ shell. They are the lightest elements in the periodic table to contain electrons in an $f$ orbital, opening up the possiblity for interesting new phenomena. On an energy scale the partially filled $4 f$ shell generally sits below the valence which tends to be comprised of $6 s$ and, in some cases, $5 d$ electrons. This explains the positioning of the rare-earth series in the fourth column of the periodic table. With the exception of Ce all of the rare-earth elements have been observed in the $3+$ charge state.

\section{$2.2 \quad$ Nitrides}

Nitride based crystalline materials have received much interest for their desirable properties. GaN, InN, and AlN are the most common recent examples and have excellent potential for use in opto-electronic devices that span the entire visible light range [14]. The availability of compatible nitride based magnetic materials would open up the possibility for a wide range of spintronic devices. The ReNs offer just that possibility. 


\section{$2.3 \quad$ Rare-Earth Nitrides}

Together, the ReNs are a class of novel crystalline materials where a rareearth element bonds with nitrogen in a 1:1 stoichiometry, leaving the rareearth element in what is effectively a $3+$ charge state. Since the 1960s, when high purity rare-earth sources were first extracted, it has been known that the ReNs all crystallize into the simple cubic rock-salt structure and that most of them order magnetically [15].

The delocalized band electrons in ReNs are, to a first approximation, comprised of rare-earth $5 d$ and $6 s$ electrons and nitrogen $2 p$ electrons. There are fourteen available electron states in the $4 f$ shell. With such a large shell it is inevitable that rare-earths across the series will have widely varying orbital and spin moments. This means that the ReN series contains fourteen chemically and structurally similar materials that each have very different magnetic properties and the potential to be used in spintronic devices based on ReN heterostructures.

Research in the twentieth century generally focused on attempts to determine whether the ReNs were metallic or semiconducting, and the measurement of their magnetic properties. Most results are detailed in the book review chapters by Hulliger [15] and Vogt et al. [16]. These experiments were performed prior to the surge of innovation in the growth of high quality nitride thin films led by the development of nitrogen plasma sources for molecular beam epitaxy of gallium nitride. Instead bulk crystal growth was typically achieved by direct reaction of nitrogen or ammonia with liquid rareearth metal (reactive arc melting), rare-earth metal shavings or sponges, or rare-earth powders; procedures that were gradually realized to be susceptible to oxygen contamination and a high concentration of nitrogen vacancies $[15,17,18,19]$. These issues make the interpretation of transport, magnetic, and optical measurements difficult. It is worth noting that more recent studies are not immune to the same shortcomings, although in general their effect is more readily appreciated [20].

In general, transport measurements indicated that the the ReNs were 
metallic or semimetallic $[21,22,23,24]$ although there are conflicting reports for many of the ReNs. For example YbN has been identified as both a semiconductor [24] and a semimetal [25]. Optical transport and absorption measurements were used to estimate the gap energies of most of the ReNs. There were reports and semi-empirical predictions of band gaps of over $2 \mathrm{eV}[26,21]$ but later studies suggest gaps of roughly $1 \mathrm{eV}[15]$. By far the most effort was expended on characterizing the magnetic structure of the ReNs and measuring the crystal fields that affect the magnetism. In short, neutron scattering and specific heat measurements across the series provided evidence for paramagnets $(\mathrm{EuN})$, ferromagnets (NdN, GdN, $\mathrm{TbN}, \mathrm{DyN}, \mathrm{TmN})$, antiferromagnets $(\mathrm{SmN}, \mathrm{YbN})$ and ferrimagnets $(\mathrm{HoN}$, ErN) $[15,16,27,28,29,22,30,31]$. The effect of oxygen impurtities on magnetism were also quantified. In GdN in particular it was reported that oxygen causes a shift from ferro- to antiferro-magnetism [32, 33, 19, 23].

Very few attempts to further detail the electronic structure of any of the ReNs are found. There are some x-ray photoemission data for the Gd pnictides $[34,35]$, and a rather advanced study of $\mathrm{YbN}$ [25]. In that study, motivated by the search for a heavy-fermion system, care was taken to avoid exposing the crystals to atmosphere and optical reflectivity performed over a large energy range $(1 \mathrm{meV}-12 \mathrm{eV})$ allowed a fairly detailed characterization of the electronic band structure. At room temperature YbN was found to be a self-compensated semimetal with an empty $4 f$ band roughly $0.2 \mathrm{eV}$ above the Fermi level.

Modern band structure calculations, appearing in concert with increased interest in spintronics, revived the ReN field with predictions that the series inludes both magnetically ordered half-metals and semiconductors [36, 13, $37,38]$.

Recent experimental work has returned focus to thin films, the growth of which has advanced considerably and is a likely prerequisite for technological applications. Leuenberger et al. recently performed a comprehensive study of GdN based on near stoichiometric nanocrystalline thin films and the use 
of a thin capping layer for protection from atmospheric degradation during ex situ characterization. They used magnetoresistance, magnetization and $\mathrm{x}$-ray photoelectron spectroscopy measurements to show that GdN is a semiconductor at room temperature and report a drop in resistivity of roughly $50 \%$ in the transition to the ferromagnetic ground state. They recognized that this could be either an intrinsic or an extrinsic effect. It has been predicted that a semiconductor-metal transition occurs in the phase transition to ferromagnetic order [36]. On the other hand, it could be that donor states mediate the conductivity and in the ferromagnetic phase transition the spin-split majority conduction band shifts to cross the donor impurity levels $[39,40,41]$.

\subsection{Hafnium Nitride}

Technically, with a full $4 f$ shell and one more $d$ electron than Lu, Hf is not a rare-earth element. Nevertheless it is similar enough to warrant further electronic structure investigations. Even with four valence electrons it is possible to grow HfN with a 1:1 stoichiometry and the same rock-salt crystal structure as the ReNs. In this case the extra valence electron raises the Fermi level into the $5 d$ bands and HfN is very clearly metallic, in contrast with the ReNs. The metallic nature of HfN is immediately obvious in the band structure calculation in Ref. [42] where the band dispersions are very similar to those calculated for LuN in Ref. [13], yet the highest occupied state lies roughly $3 \mathrm{eV}$ higher. Experiments measuring the HfN crystal structure and partial density of states are reported in Sections 5.2.2 and 8.1, respectively. 


\section{Chapter 3}

\section{Theoretical Background}

In principle the observable properties of any crystalline system can be extracted by writing down and solving the many-body Dirac equation. For most observables, and most systems, the time independent many-body Schrödinger equation

$$
\mathcal{H} \Psi=E \Psi
$$

is sufficient. Here the Hamiltonian, $\mathcal{H}$, contains kinetic and potential terms for each particle in the system and operates on the many-body wavefunction, $\Psi$, to detemine the total energy, $E$.

For systems with more than a few particles the Schrödinger equation is very difficult to solve, even numerically, so simplifications are introduced, usually beginning with the Born-Oppenheimer approximation. The ions in a crystalline material are many orders of magnitude more massive than the electrons, so they can be thought of as stationary point particles surrounded by rapidly moving electrons. Under this assumption the kinetic energy of the ions is negligible and the Coulomb interaction between the ions is a constant and can be ignored for the purpose of finding the ground state wavefunction. The total Hamiltonian of the electons, $i$, is then

$$
\mathcal{H}=-\sum_{i} \frac{\hbar^{2}}{2 m_{i}} \nabla_{\mathbf{r}_{i}}^{2}-\sum_{i} \sum_{I} \frac{Z_{I} e^{2}}{\left|\mathbf{R}_{I}-\mathbf{r}_{i}\right|}+\frac{1}{2} \sum_{i} \sum_{j(j \neq i)} \frac{e^{2}}{\left|\mathbf{r}_{j}-\mathbf{r}_{i}\right|}
$$


The three terms, from left to right, are the kinetic $(T)$, external $\left(V_{\text {ext }}\right)$, and electron-electron Coulomb $(U)$ operators. For this Hamiltonian, $V_{\text {ext }}$ is simply the potential due to the ions, $I$. However, in general it can be extended to include any external potential, hence the name. Solving this equation to obtain the many-body wavefunction

$$
\Psi=\Psi\left(\left\{\mathbf{r}_{i}\right\}\right), \quad\left\{\mathbf{r}_{i}\right\}=\mathbf{r}_{1}, \mathbf{r}_{2}, \ldots \mathbf{r}_{N}
$$

is then enough to completely determine the observable properties of the ground state of a condensed matter system. If only we were clever enough! It is still simply too computationally expensive to numerically solve a manybody problem like this for any realistic system.

Further progress can only be made with even more severe approximations. Historically, the two most successful approaches to this problem have been the Hartree-Fock approximation (HFA) and the density functional theory (DFT). The HFA was developed in the early days of quantum mechanics and is based on a guess of the form of the many-electron wavefunction. DFT is a more recent approach, based on the Honenberg-Kohn theorems from the nineteen sixties, which proved that the ground state density can be used as the basic variable, rather than the many-electron wavefunction.

In this thesis the HFA, detailed in Section 3.1, is used to calculate the single atom energy levels needed to simulate the multiplet effects seen in x-ray spectroscopy, which are introduced in Section 3.3. Advanced formulations of DFT, detailed in Section 3.2, are used to calculate the electronic band structure of the ReNs, which are introduced in Section 3.4.

\subsection{The Hartree-Fock Approximation}

The HFA is based on a guess of the form of the many-electron wavefunction. Perhaps the simplest such guess is the Hartree approximation (HA)

$$
\Psi^{H A}\left(\left\{\mathbf{r}_{i}\right\}\right)=\phi_{1}\left(\mathbf{r}_{1}\right) \phi_{2}\left(\mathbf{r}_{2}\right) \ldots \phi_{N}\left(\mathbf{r}_{N}\right),
$$


where the many-electron wavefunction is a product of single electron wavefunctions, $\phi_{1}\left(\mathbf{r}_{1}\right)$. Written in this way the many-electron wavefunction is appropriate for a system where where the electrons behave as independent particles moving in the average field of all of the other electrons. The HFA was constructed to improve upon the HA by explicitly incorporating the notion that electrons are Fermions and so the overall wavefunction is antisymmetric - exchanging any two electrons will result in a sign change of the wavefunction. The inclusion of antisymmetry in the HFA is important because it tends to favour wavefunctions that keep electrons with similar quantum numbers apart, thereby reducing the overall Coulomb energy. However, it still does not include the full correlations amongst electrons. The Slater determinant, a determinant of the one-partical wavefunctions, trivially enforces antisymmetry

$$
\Psi^{H F A}\left(\left\{\mathbf{r}_{i}\right\}\right)=\frac{1}{\sqrt{N !}}\left|\begin{array}{ccc}
\phi_{1}\left(\mathbf{r}_{1}\right) & \phi_{1}\left(\mathbf{r}_{2}\right) & \ldots \\
\phi_{2}\left(\mathbf{r}_{1}\right) & \phi_{2}\left(\mathbf{r}_{2}\right) & \ldots \\
\vdots & \vdots & \ddots
\end{array}\right| .
$$

A variational approach [43] can be used to find the single-particle HartreeFock equations

$$
\begin{gathered}
{\left[-\frac{\hbar^{2}}{2 m_{e}} \nabla_{\mathbf{r}}^{2}-\frac{Z e^{2}}{|\mathbf{r}|}+e^{2} \sum_{j(j \neq i)}\left\langle\phi_{j}\left|\frac{1}{\left|\mathbf{r}-\mathbf{r}^{\prime}\right|}\right| \phi_{j}\right\rangle\right] \phi_{i}(\mathbf{r})} \\
-e^{2} \sum_{j(j \neq i)}\left\langle\phi_{j}\left|\frac{1}{\left|\mathbf{r}-\mathbf{r}^{\prime}\right|}\right| \phi_{i}\right\rangle \phi_{j}(\mathbf{r})=\epsilon_{i} \phi_{i}(\mathbf{r}) .
\end{gathered}
$$

Here, we have set $V_{\text {ext }}=-\frac{Z e^{2}}{|\mathbf{r}|}$ to the ionic potential of a single atom, as will be needed to calculate atomic energy levels in Section 3.3. The last term on the left hand side of the equation is known as the exchange term and is what differentiates the HFA from the HA.

\subsubsection{Solving the single particle equation}

The problem of determining the ground state of the system is now simplified to solving the eigenvalue problem of Eq. 3.6 for the single electron wave- 
functions. Since the Coulomb and exchange terms for one electron both depend on the other single electron wavefunctions, the equation must usually be solved iteratively, until the system of wavefunctions is self-consistent. Ref. [43] provides a overview of how this is generally done. First, a guess (or result of a previous iteration cycle) for the wavefunctions is obtained. This is used to calculate the terms on the left hand side of Eq. 3.6. Solving the equation results in a new set of wavefunctions. These are combined with the previous wavefunctions in some way and the cycle repeated. After each iteration the energy can be calculated. Once the difference in energy between the previous two iterations is smaller than some predetermined threshold the system is considered to have self-consistently converged.

\subsection{Density Functional Theory}

While the HFA is based on a guess of the form of the ground state wavefunction, DFT is based on the Hohenberg-Kohn theorems which provide a basis for determining the electronic structure of a condensed matter system through the electron density, rather than through the many-body wavefunction $[44,45]$.

Essentially, Hohenberg and Kohn showed that there is a one-to-one correspondence between the electronic density and the external potential of any system. Subsequently they defined an energy functional (of the density) and proved that the ground state density minimizes this energy [43]. As is outlined below, the use of the density as the key variable produces a framework for mapping the many-body problem onto a single-body problem.

The density, $n(\mathbf{r})$, which depends on only three parameters $(\mathbf{r})$, can be calculated easily from the many-body wavefunction, which depends on $3 N$ parameters

$$
n(\mathbf{r})=N \int \Psi^{*}\left(\mathbf{r}, \mathbf{r}_{2}, \ldots \mathbf{r}_{N}\right) \Psi\left(\mathbf{r}, \mathbf{r}_{2}, \ldots \mathbf{r}_{N}\right) d \mathbf{r}_{2} \ldots d \mathbf{r}_{N}
$$

Since the external potential defines not just the density but also the wave- 
function it must be the case that the wavefunction is a functional of the density

$$
\Psi_{0}=\Psi\left[n_{o}\right]
$$

So the energy, which is still specified by the Hamiltonian in Eq. 3.2, is given by

$$
\begin{aligned}
E_{0}\left[n_{0}\right] & =\left\langle\Psi_{0}\left|T+V_{\text {ext }}+U\right| \Psi_{0}\right\rangle \\
& =T\left[n_{0}\right]+V_{\text {ext }}\left[n_{0}\right]+U\left[n_{0}\right] .
\end{aligned}
$$

While ignoring difficulties involved with writing down the terms in the energy functional there are some general results which allow further progress. Consider a system of non-interacting fermionic particles, not necessarily electrons (represented by the subscript $s$ ). In this case the electron-electron interaction term, $U$, in the energy functional disappears, so

$$
E_{s}\left[n_{s}\right]=T_{s}\left[n_{s}\right]+V_{s}\left[n_{s}\right]
$$

Since the particles involved in this equation are not interacting they can be represented exactly as a Slater determinant (Eq. 3.5) and the density is given by

$$
n(\mathbf{r})=\sum_{i}\left|\phi_{i}\right|^{2}
$$

A variational approach to minimizing the non-interacting particle energy results in what are known as the Kohn-Sham equations [43]

$$
\left[-\frac{\hbar^{2}}{2 m} \nabla_{\mathbf{r}}^{2}+V_{s}(\mathbf{r}, n(\mathbf{r}))\right] \phi_{i}(\mathbf{r})=\epsilon_{i} \phi_{i}(\mathbf{r}) .
$$

The system of Kohn-Sham equations describe an eigenvalue problem, very similar to the Hartree-Fock equation (3.6). Since $V_{s}$ depends on $n_{s}$, which depends on $\phi_{i}$, which in turn depend on $V_{s}$, the problem must be solved in a self-consistent way (i.e., iteratively). The techniques used to achieve this are generally very similar to that described for the HFA in Section 3.1.1. 
The Kohn-Sham equations were derived for a completely general potential, $V_{s}$. By specifying that

$$
V_{s}=\left(T-T_{s}\right)+V_{\mathrm{ext}}+U
$$

the potentials in Eqs. 3.9 and 3.10 are identical and therefore must have the same density! This means that a solution to the Kohn-Sham equations, based on a set of fictitious non-interacting particles (quasiparticles), will reproduce the density of the real system. In this way the problem of obtaining the ground state of the original system of interacting electrons in the presence of a real external potential has been converted to a problem of obtaining the ground state of a system of quasiparticles in the presence of an effective potential; and it has been achieved in a way that reproduces the density (and therefore all observables for which a functional is known) of the real system.

\subsubsection{Exchange-correlation functionals}

Of course the effective potential which, in its most general form, can be written as

$$
V_{s}\left[\mathbf{r}, n_{s}(\mathbf{r})\right]=V_{\mathrm{ext}}(\mathbf{r})+e^{2} \int \frac{n_{s}\left(\mathbf{r}^{\prime}\right)}{\left|\mathbf{r}-\mathbf{r}^{\prime}\right|} d \mathbf{r}^{\prime}+\frac{\delta E_{X C}\left[n_{s}(\mathbf{r})\right]}{\delta n_{s}(\mathbf{r})},
$$

must still be specified. It is the third term, the exchange and correlation potential (defined in terms of the exchange and correlation energy functional) that is not known exactly. Inventing accurate approximations to this term is one of the key challenges in DFT.

A number of approximations have been suggested, all of which are common in that they do not seem to be particularly realistic (although the results of numerical calculations are generally in remarkable agreement with experiment). Two commonly used forms for $E_{X C}$ are the local density approximation (LDA) and the generalised gradient approximation (GGA). The LDA functional is "local" in that it depends only on the density at each point in space (and not, for example, on the gradient of the density, which is also 
included in the GGA). In materials where the majority- and minority-spins are not degenerate (and therefore electron spin is important) the LDA is trivially generalized to the local spin density approximation (LSDA)

$$
E_{X C}^{\mathrm{LSDA}}\left[n_{\uparrow}, n_{\downarrow}\right]=\int \epsilon_{X C}\left[n_{\uparrow}, n_{\downarrow}\right] n(\mathbf{r}) d^{3} \mathbf{r}
$$

which is the basis for the functionals used in this thesis. The single parameter in the equation is the energy density, $\epsilon_{X C}$, which has been accurately determined for a range of densities by studies of the free electron model.

\subsection{Atomic Multiplets}

The Re $4 f$ levels can be directly probed by exciting a $3 d^{10} 4 f^{n} \rightarrow 3 d^{9} 4 f^{n+1}$ optical transition. The experimental details of this technique are described fully in Section 4.4.1. Briefly, these transitions probe what are known as the Re $\mathrm{M}_{4,5}$ edges, which have binding energies of roughly $1200 \mathrm{eV}$ - in the soft x-ray range. The similarities between the Re and ReN M-edge x-ray absorption (see Fig 8.4) indicate that the transitions are mostly independent of bonding enviroment are thus largely atomic in nature, so a theoretical understanding must begin at this starting point.

The theory of atomic electronic structure and resulting intra-atomic transitions is well understood. A full treatment of the many-electron atom can be found in the canonical work by Cowan [46]. For more recent results specific to calculating the M-edge X-ray absorption spectra of $\mathrm{Re}^{3+}$ ions, see Thole et al. [47] and Goedkoop et al. [48]. It is shown in these references that the $3 d^{10} 4 f^{n} \rightarrow 3 d^{9} 4 f^{n+1}$ transition is complicated by multiplet effects - a strong overlap of the core and valence wavefunctions means that a single particle description of the transition is not valid and electron-electron interactions become important [49].

The general process used to calculate the $\mathrm{Re}^{3+}$ M-edge transition probabilities is as follows. First, the initial and final state wavefunctions of the Re ion are calculated using the HFA. From the wavefunctions some key terms 
- the Slater-Coulomb integrals and spin-orbit couplings - are extracted. These parameters are used to calculate the energy levels of each possible multi-electron configuration in the unfilled $3 d$ and $4 f$ shells. Finally, the electric dipole transition probabilities are calculated from these configurations.

\subsubsection{The atomic Hamiltonian}

The Hamiltonian that describes a multi-electron atom follows directly from Eq. 3.2. It contains three terms: the electron kinetic energy, the electronnucleus Coulomb attraction, and the electron-electron Coulomb repulsion. The first two terms are the same for electrons in the same shell; this average energy will be ignored in what follows. In heavier atoms, like the rare-earths, relativistic effects become important. Treated as a perturbation this adds a spin-orbit coupling term to the Hamiltonian. ${ }^{1}$ It is the electron-electron and spin-orbit terms that determine the relative energy levels of different possible open-shell electron configurations

$$
\mathcal{H}=\frac{1}{2} \sum_{i} \sum_{j(j \neq i)} \frac{e^{2}}{\left|\mathbf{r}_{j}-\mathbf{r}_{i}\right|}+\sum_{i} \zeta\left(\mathbf{r}_{\mathbf{i}}\right)\left(\boldsymbol{l}_{\boldsymbol{i}} \cdot \boldsymbol{s}_{\boldsymbol{i}}\right) .
$$

This is a many-body problem, best dealt with under the HFA. In the following we will provide just an overview of the key results. Fairly accessible reviews of the state of the art in can be found in Refs. [49, 50], and a complete treatment was authored by Cowan [46].

It can be shown that the relative energy of a given electron configuration depends only its term symbol (Section 3.3.2) [49], in which case the electron-electron term of Eq. 3.16 can be written in terms of Slater-Condon integrals [49]

$$
\left\langle{ }^{2 S+1} L_{J}\left|\sum_{i} \sum_{j(j \neq i)} \frac{e^{2}}{\left|\mathbf{r}_{\mathbf{i j}}\right|}\right|^{2 S+1} L_{J}\right\rangle=\sum_{k} f_{k} F^{k}+\sum_{k} g_{k} G^{k} .
$$

\footnotetext{
${ }^{1}$ There are other first order terms in the perturbation that are not important here. For discussion see Ref. [46].
} 


\begin{tabular}{|c|cccccccccc|}
\hline & $F_{f}^{2}$ & $F_{f}^{4}$ & $F_{f}^{6}$ & $\zeta_{f}$ & $F_{d}^{2}$ & $F_{d}^{4}$ & $\zeta_{d}$ & $G_{f d}^{1}$ & $G_{f d}^{3}$ & $G_{f d}^{5}$ \\
\hline $3 d^{10} 4 f^{7}$ & 11.60 & 7.28 & 5.24 & 0.20 & & & & & & \\
$3 d^{9} 4 f^{8}$ & 12.16 & 7.64 & 5.50 & 0.23 & 7.77 & 3.63 & 12.36 & 5.56 & 3.26 & 2.25 \\
\hline
\end{tabular}

Table 3.1: The ground and excited state $\mathrm{Gd}^{3+}$ Coulomb and exchange integrals and spin-orbit coefficients. The values for all Re ions are tabulated in Ref. [47]

On the right the angular (lower case) and radial (upper case) components of the Coulomb $\left(f_{k} F^{k}\right)$ and exchange $\left(g_{k} G^{k}\right)$ energy have been isolated $[49,50]$. The Coulomb sum is constrained by the $3 j$ symbol present in the calculation of the radial component [46]. For the $4 f$ orbital $k=0,2,4,6$. For the exchange energy $k=\left|l-l^{\prime}\right|,\left|l-l^{\prime}\right|+2, \ldots l+l^{\prime}$, where $l$ and $l^{\prime}$ are the orbital angular quantum numbers of the two shells that the exchange interaction is between.

The Slater-Coulomb integrals and the radial spin-orbit coupling parameter, $\zeta\left(\mathbf{r}_{\mathbf{i}}\right)$, can all be extracted once the HFA has been used to calculate the multi-electron wavefunction. For example, the parameters for the initial and final states of the $4 f^{7} \rightarrow 3 d^{9} 4 f^{8}$ transition in $\mathrm{Gd}^{3+}$ are listed in Table 3.1. Note that the $F$ and $G$ have all been scaled to $80 \%$ of their calculated value, an empirical adjustment required in order to reach agreement with experiment $[49,47]$.

\subsubsection{Coupling schemes}

In the Hamiltonian used to calculate the multiplet energy levels (Eq. 3.17) two standard ways of coupling together individual angular momenta are used: $L S$ and $j j$ coupling. In $L S$ coupling, also known as Russell-Saunders coupling, the individual orbital moments, $l_{i}$, are coupled together to form the total orbital momentum, L. A similar approach is used for $S$. $L$ and $S$ are then coupled to form the total angular momentum, $J$. In $j j$ coupling the individual orbital, $l_{i}$, and spin, $s_{i}$ moments are coupled to form $j_{i}$. The $j_{i}$ 
are then coupled to form the total angular momentum, $J$.

Under $L S$ coupling $L, S$, and $J$ all commute with the Coulomb repulsion operator. On the other hand the spin-orbit operator commutes with $j_{i}$ and $J$. This means that in systems where the Coulomb term dominates the spin-orbit coupling an $L S$ basis is closest to being diagonal, while in systems where the spin-orbit coupling dominates a $j j$ basis is simpler. Note that pure coupling schemes are only used for convenience of representation; modern computational frameworks fully diagonalize the Hamiltonian matrix. In this case, known as intermediate coupling, it does not matter which coupling representation is chosen.

When the interacting orbitals are $2 p, 3 d$, or $4 f$ in nature $L S$ coupling is most applicable (so this is the representation most commonly used to describe Re ions). The $L S$ coupled momenta are commonly represented as term symbols

$$
{ }^{2 S+1} L_{J},
$$

which contain all information on the angular momentum of the system. In the term symbol $S$ and $L$ are the total spin and orbital moments, $2 S+1$ is the multiplicity of the term, and $J$ is the total angular momentum. $L$ is generally written as $\{S, P, D, F, G, \ldots\}$ for total moments of $\{0,1,2,3,4, \ldots\}$. A combination of $S$ and $L$ defines a term, which would uniquely specify the energy if there were no spin-orbit coupling [49]. For each term $J$ is restricted to

$$
|L-S| \leq J \leq L+S
$$

As more electrons, $n$, are included in a shell, or as the orbital moment, $l$, of the shell gets larger, the number of possible ways for the electrons to occupy the shell, $N$, grows combinatorically

$$
N=\left(\begin{array}{c}
2(2 l+1) \\
n
\end{array}\right)
$$

This makes multiplet calculations for the $4 f$ orbital increasingly complex. For example, seven electrons can half-fill the $\mathrm{Gd}^{3+} 4 f$ shell in 3432 different ways (see Table 3.2). 


\subsubsection{Hund's rules}

The ground state term symbol for a partially filled shell can be easily determined in $L S$ coupling (weak spin-orbit coupling) by following a few simple rules, known as Hund's rules:

1. maximize spin moment; then

2. maximize orbital moment; then finally

3. $J=|L-S|$ or $|L+S|$ for less or more than half-filled shells.

The rules are listed in order of priority — the orbital moment is maximized subject to the constraint that spin moment remains maximized. Then the total moment, $J$, is chosen. The rules can be interpreted in terms of the HFA. Rules one and two result from minimising the Coulomb energy subject to an antisymmetric wavefunction. Rule three results from spin-orbit coupling which breaks the degeneracy in $J$.

Hund's rules are accurate enough to correctly specify the ground state term for all of the $\mathrm{Re}^{3+}$ ions. The ground state, and its energy relative to the first excited state (calculated under the HFA), is shown in Table 3.2.

\subsubsection{Multiplet dipole transitions}

The final step in calculating the Re M-edge x-ray absorption spectrum is to determine the multiplet optical (dipole) transition probabilities from the initial state, $4 f^{n}$, to the final state, $3 d^{9} 4 f^{n+1}$. The initial state can often be taken as just the ground state multiplet since $k_{B} T(\sim 25 \mathrm{meV}$ at $295 \mathrm{~K})$ is usually significantly less than the first excited multiplet state (see Table 3.2). ${ }^{2}$

\footnotetext{
${ }^{2}$ Numerical calculations often (correctly) incorporate a full Boltzmann distribution of the initial state multiplets at a given temperature. Typically the calculated spectra are no different from those that incorporate only the ground state multiplet. This simplifies calculations of the complicated $4 f$ transitions. However, when crystal field effects are incorporated the ground state approximation can break down. Measurements of the SmN magnetic moment showed that this is the case for $\mathrm{Sm}^{3+}$ in $\mathrm{SmN}$, see Section 7.2.1.
} 


\begin{tabular}{|c|c|c|c|c|c|c|}
\hline \multirow[t]{2}{*}{$\operatorname{Re}^{3+}$} & \multicolumn{4}{|c|}{$4 f^{n}$} & \multirow[t]{2}{*}{$T$} & \multirow{2}{*}{$\frac{3 d^{9} 4 f^{n+1}}{M}$} \\
\hline & $n$ & $N$ & ${ }^{2 S+1} \mathrm{~L}_{J}$ & $\Delta E(\mathrm{eV})$ & & \\
\hline $\mathrm{La}$ & 0 & 1 & ${ }^{1} \mathrm{~S}_{0}$ & - & 3 & 20 \\
\hline $\mathrm{Ce}$ & 1 & 14 & ${ }^{2} \mathrm{~F}_{2.5}$ & -0.305 & 53 & 107 \\
\hline $\operatorname{Pr}$ & 2 & 91 & ${ }^{3} \mathrm{H}_{4}$ & -0.290 & 176 & 386 \\
\hline $\mathrm{Nd}$ & 3 & 364 & ${ }^{4} \mathrm{I}_{4.5}$ & -0.255 & 414 & 977 \\
\hline $\mathrm{Pm}$ & 4 & 1,001 & ${ }^{5} \mathrm{I}_{4}$ & -0.201 & 773 & 1,878 \\
\hline $\mathrm{Sm}$ & 5 & 2,002 & ${ }^{6} \mathrm{H}_{2.5}$ & -0.143 & 946 & 2,725 \\
\hline $\mathrm{Eu}$ & 6 & 3,003 & ${ }^{7} \mathrm{~F}_{0}$ & -0.056 & 213 & 3,106 \\
\hline $\mathrm{Gd}$ & 7 & 3,432 & ${ }^{8} \mathrm{~S}_{3.5}$ & -3.892 & 1,077 & 2,725 \\
\hline $\mathrm{Tb}$ & 8 & 3,003 & ${ }^{7} \mathrm{~F}_{6}$ & -0.269 & 651 & 1,878 \\
\hline Dy & 9 & 2,002 & ${ }^{6} \mathrm{H}_{7.5}$ & -0.452 & 226 & 977 \\
\hline Ho & 10 & 1,001 & ${ }^{5} \mathrm{I}_{8}$ & -0.654 & 61 & 386 \\
\hline Er & 11 & 364 & ${ }^{4} \mathrm{I}_{7.5}$ & -0.829 & 16 & 107 \\
\hline $\mathrm{Tm}$ & 12 & 91 & ${ }^{3} \mathrm{H}_{6}$ & -0.662 & 4 & 20 \\
\hline $\mathrm{Yb}$ & 13 & 14 & ${ }^{2} \mathrm{~F}_{3.5}$ & -1.281 & 1 & 2 \\
\hline
\end{tabular}

Table 3.2: Properties of the $\operatorname{Re}^{3+}$ multiplets. Each $\mathrm{Re}^{3+}$ ion has $n 4 f$ electrons which can fill the $4 f$ shell in $N$ different configurations. Term symbols can be calculated for each configuration. The ground state term symbol, ${ }^{2 S+1} \mathrm{~L}_{J}$, can be calculated using Hund's rules, or numerically using Cowan's rcg program. The energetic separation, $\Delta E$, between the ground state and the first excited state was also extracted from the numerical calculations. There are $T$ different dipole allowed transitions from the ground state multiplet to the $M$ different $3 d^{9} 4 f^{n+1}$ final state multiplets. 
On the other hand the final state is comprised of all of the possible $3 d^{9} 4 f^{n+1}$ multiplets.

The transition probabilities between the initial and final states can then be calculated using Fermi's golden rule (see Section 4.4.1), which incorporates the dipole selection rule. Interpreted in the language of term symbols the dipole selection rule states that

$$
\Delta J= \begin{cases}0, \pm 1 & \text { if } J \neq 0 \\ \pm 1 & \text { if } J=0\end{cases}
$$

This severely restricts the number of possible final states for an optical transition. Even so, there are often still hundreds of significant transitions (lines) in $\mathrm{Re}^{3+}$ M-edge x-ray absorption spectra. The number of final state multiplets, and the number of allowed transitions from the ground state multiplet of the initial state to the final state multiplets of each of the $\mathrm{Re}^{3+}$ ions, is listed in Table 3.2 .

\subsubsection{Software}

The software needed to calculate multiplet effects is readily available. The code was written by Cowan and is reviewed in Ref. [46]. The general technique for calculating multiplet energy levels under the HFA relies on a number of individual steps, each of which is a performed by a different program:

- rcn uses the HFA to calculate the atomic many-body wavefunction for both the ground and excited states;

- rcn2 extracts the Coulomb, exchange, and spin-orbit terms from the many-body wavefunctions;

- $r c g$ uses these parameters to calculate the energy levels for each possible term and the dipole allowed transitions between the ground and excited state terms. 
From the output or these programs atomic x-ray absorption spectra can be extracted. The atom-based approach can be extended to calculate transitions for atoms in various crystal fields, by incorporating results based on breaking the symmetry of the atomic Hamiltonian. In practice this is achieved by inputing the calculated multiplet states from rcg into racah, a program written by Thole, which is used to include crystal field effects in the calculation. These programs are freely available as bundles. TTMULT [49] and Missing [51] are the two program bundles used in this thesis. Missing was used to calculate the ground and excited state energy levels presented in Table 3.2.

\subsection{Electronic Band Structure}

The electronic band structure of the ReNs is calculated using a specialised formulation of the basic DFT which was introduced in Section 3.2. Key choices that are made are in the form of the exchange-correlation functional, the external potential, and the basis set. These choices are particularly important in calculations of the ReNs because of the magnetic nature of the ground state and the strongly correlated nature of the electrons in the $4 f$ orbitals. In fact the uncertainty surrounding the correctness of the electronic structure calculated using these choices underpins the theoretical interest in the ReNs.

The code base that was used in this thesis was written by a group led by M. van Schilfgaarde [52]. The LSDA $+U$ extension was implemented by W. R. L. Lambrecht. The full details of the technique, as applied to the ReNs, are outlined in Refs. [53] and [13]. Band structure calculations in this thesis rely heavily on these papers. Below, an outline of the choice of the exchange-correlation functional will be made. 


\subsubsection{LSDA $+U$}

The exchange-correlation functional most typically used in band structure calculations of spin polarized materials is the LSDA. However, in orbitals where electron-electron interactions are important, like the Re $4 f$ orbital, the LSDA fails to correctly calculate the electronic density because the exchange energy is not well approximated [54].

The Hamiltonian that is often used to qualitatively describe these strongly correlated systems is provided by the Hubbard model,

$$
H=\sum_{i} U n_{f, i, \uparrow} n_{f, i, \downarrow}
$$

where $U$ is the Hubbard parameter and $n_{f, i, \sigma}$ is the number of $4 f$ electrons at site $i$ with spin $\sigma$ ( $\uparrow$ or $\downarrow$ ). It is generally accepted that strongly correlated systems are well described by the Hubbard model [55].

By considering both the LSDA and the Hubbard model the following exchange-correlation energy functional was proposed $[55,56]$

$$
E^{L S D A+U}\left[n^{\sigma},\left\{\eta^{\sigma}\right\}\right]=E^{L S D A}\left[\rho^{\sigma}\right]+E^{U}\left[\left\{\eta^{\sigma}\right\}\right]-E^{\mathrm{dc}}\left[\left\{\eta^{\sigma}\right\}\right],
$$

where $n^{\sigma}$ is the spin-resolved density and $\left\{\eta^{\sigma}\right\}$ is the density matrix for the correlated electrons in the $4 f$ shell, as defined in Ref. [56]. The first of the three terms is the spin resolved generalization of the the standard LDA functional, $E^{L S D A}(3.15)$. The other two terms are the Hubbard- $U$ term, and the double counting correction term (inserted to ensure that $E^{L S D A+U}=E^{L S D A}$ in the absence of orbital polarizations). The functionals are not reproduced here but can be shown to result, through (3.14), in the following potential addition to $V_{\mathrm{XC}}$

$$
\begin{aligned}
V_{m m^{\prime}}^{\sigma} & =\sum_{m^{\prime \prime} m^{\prime \prime \prime}}\left[\left\langle m, m^{\prime \prime}\left|V_{e e}\right| m^{\prime}, m^{\prime \prime \prime}\right\rangle n_{m^{\prime \prime} m^{\prime \prime \prime}}^{-\sigma}\right. \\
& +\left(\left\langle m, m^{\prime \prime}\left|V_{e e}\right| m^{\prime}, m^{\prime \prime \prime}\right\rangle\right. \\
& \left.-\left\langle m, m^{\prime \prime}\left|V_{e e}\right| m^{\prime \prime \prime}, m^{\prime}\right\rangle n_{m^{\prime \prime} m^{\prime \prime \prime}}^{\sigma}\right] \\
& -U\left(\operatorname{Tr}\left(n_{m m^{\prime}}^{\sigma}+n_{m m^{\prime}}^{-\sigma}\right)-\frac{1}{2}\right)+J\left(\operatorname{Tr}\left(n_{m m^{\prime}}^{\sigma}\right)-\frac{1}{2}\right),
\end{aligned}
$$


where $V_{e e}$ are the screened Coulomb interactions between the $4 f$ electrons and $U$ and $J$ are functions of the screened Slater-Coulomb integrals - the very same parameters introduced for the HFA in Section 3.3.1. In fact the Coulomb integrals also appear when $V_{e e}$ is written in a basis of spherical harmonics (cf. Eq. 3.17) [56]

$$
\left\langle m, m^{\prime \prime}\left|V_{e e}\right| m^{\prime}, m^{\prime \prime \prime}\right\rangle=\sum_{k} f_{k} F^{k} .
$$

The values for $U$ and $J$ can be written in terms of Coulomb integrals. For the Re $4 f$ orbital $F^{0}$ is heavily screened, while the ratios between $F^{2}, F^{4}$, and $F^{6}$ are known to be independent of the element in question [13]

$$
\begin{aligned}
& U_{f}=c F^{0}, \\
& J_{f}=\left(286 F^{2}+195 F^{4}+250 F^{6}\right) / 6435 .
\end{aligned}
$$

Thus the LSDA $+U$ exchange-correlation functional provides a strong link back to the atomic formulation of the HFA. In a way this is not surprising since the Hubbard- $U$ corrections were introduced to deal with the atomiclike nature of the Re $4 f$ orbital. This correspondence means that direct measurements of the Re $4 f$ orbital by x-ray spectroscopy can be used to help determine the correct parameters to use in the band structure calculations. Since it is strongly screened, $U_{f}$ is typically determined experimentally, by tuning the value until agreement is reached with XPS measurements. On the other hand, $J_{f}$ can be extracted straight from Hartree-Fock calculations of the Coulomb integrals.

In calculations of the ReNs Hubbard- $U$ shifts are also applied to the Re $5 d$ orbitals, although the underlying physical justification is quite different [13]. ${ }^{3}$ It is a well known flaw of DFT calculations that they underestimate the magnitude of semiconductor band gaps, because long-range Coulomb screening is not correctly dealt with by the LSDA exchange-correlation functional. The use of $U_{d}$ adds a potential to the Hamiltonian which acts in opposite senses

\footnotetext{
${ }^{3}$ The justification may be different but the $U_{f}$ and $U_{d}$ parameters are related in that they are both strongly dependent on the strength of the long-range Coulomb screening.
} 
for occupied and unoccupied states, pushing them away from the Fermi level in both cases. Since the CBM is mostly Gd $5 d$ in nature, a positive $U_{d}$ acts to increase the size of the splitting between occupied and unoccupied states. The value of $U_{d}$ can then be tuned to reach agreement with experiment as is done in Section 6.5. 



\section{Chapter 4}

\section{Experimental Band Structure Probes}

Four primary experimental techniques were used to probe the electronic and magnetic structure of the ReNs: transport, magnetometry, optical transmission, and x-ray spectroscopy. Experimental details, and an outline of how each experiment provides information about the electronic structure of a material, are provided in this chapter.

\subsection{Transport}

Temperature dependent transport measurements probe the density of states near the Fermi level. From a simplified perspective, samples are often classified according to the slope of their resistivity measured as a function of temperature. A positive slope implies that temperature mediated scattering is the dominant effect and a metallic sample is likely. However, it is possible for a degenerately doped semiconductor to show the same behaviour ${ }^{1}$. A negative slope often implies a temperature dependent activation of charge

\footnotetext{
${ }^{1} \mathrm{~A}$ degenerately doped semiconductor has enough imperfections that the state becomes band-like
} 
carriers, in which case a semiconducting sample is likely. ${ }^{2}$ If the sample is a semiconductor the resistivity can generally be fitted to one of the following two formulae. An activated semiconductor varies as

$$
\rho \sim e^{\frac{E_{A}}{k_{B} T}}
$$

where the activation energy, $E_{A}$, is the energy gap between the Fermi level and the nearest delocalized states. If, however, conduction in the semiconductor is mediated primarily by localized states at the Fermi energy, as can be the case when a large number of impurities are present in a sample, then the resistivity takes the form described by variable range hopping [57]

$$
\rho \sim e^{\left(\frac{T_{0}}{T}\right)^{\frac{1}{4}}}
$$

In magnetic materials the transition from the paramagnetic state to the magnetically ordered ground state can affect sample resistivity through at least two mechanisms. An increase in magnetic order can decrease the scattering rate and hence the resistivity. It is also possible that exchange splitting in the valence and conduction bands $(\mathrm{VB} \text { and } \mathrm{CB})^{3}$ will change the distance between the Fermi level and the nearest delocalized states, affecting the resistivity. These effects will result in deviations from expected metallic or semiconducting behaviour as has previously been observed in GdN [41].

In the present study the resistivity of numerous samples were measured while still under vacuum in the growth chamber. A standard two-terminal technique was used during these in situ experiments to measure the resistance across substrates prepared with two thin metal contacts on the surface. Resistivity was monitored during growth and used to obtain real time information about the deposition of the film and capping layers. When insulating capping layers are chosen, temperature dependent transport measurements

\footnotetext{
${ }^{2}$ There are other effects that can have a negative slope as a function of temperature, for example weak localization.

${ }^{3}$ Note also the related acronyms for the valence band maximum (VBM) and the conduction band minimum (CBM).
} 
are possible before and after the growth of the capping layer. These measurements are performed in order to obtain low temperature resistivity data and also to help confirm that the capping layer has not altered the sample. Making the same measurement after venting to atmosphere is a useful method for ensuring that the film has not oxidized, and is detailed in Section 5.1.4. The sample was cooled by flowing cold nitrogen gas through a pipe anchored to a copper substrate holder and measured by a PT100 thermometer embedded in the copper block.

The ex situ temperature dependent measurements were all four-terminal in nature. For the samples with high resistivity the current and voltage leads were connected to the same metal contact, while for samples that were conducting enough for the contact resistances to become important a current path was scribed on the film and the four leads were attached by pressing indium contacts to the surface. In these measurements thermoelectric voltages in the connections between the measurement equipment (at room temperature) and the sample can cause errors. Averaging the voltage measured with the current flowing in both directions removes this offset.

Temperature dependent measurements were made in a Janis Research SHI-4 closed-cycle cryostat and also in a custom made liquid bath cryostat. In the closed-cycle cryostat the sample was fixed to the cold finger by General Electric (GE) 7031 varnish — which has the useful property that a thin layer is thermally conducting and electrically insulating — and the sample space pumped to a pressure below $10^{-4}$ mbar to thermally insulate the system. The temperature was measured by two independent LakeShore DT-670 Silicon diodes, one of which is attached to the cold finger close to the sample, and controlled by a LakeShore 340 Temperature controller. In the liquid bath cryostat the inner chamber, which holds the sample, is insulated from atmosphere by evacuating the vacuum jacket that surrounds it. The sample is immersed in either liquid nitrogen or liquid helium and temperature dependent data are obtained by measuring the resistance as the liquid boils away and the system warms. In this system the temperature is measured 
by a calibrated silicon diode thermometer attached to the surface of the film with GE varnish. The system is described in detail in Ref. [58]. In both cryostats data were obtained from $300 \mathrm{~K}$ down to below $5 \mathrm{~K}$.

\subsection{Magnetometry}

Magnetization measurements provide details on the magnitude and ordering of magnetic moments in a material. The magnetic response can be measured as a function of an applied field or temperature. Usually the magnetization, $M$, of a material is written as a function of an applied field, $H$

$$
M=\chi H .
$$

In general the susceptibility, $\chi$, is temperature and magnetic field dependent. In a material where the individual atomic moments interact with each other, but above any ordering temperature (i.e., in the paramagnetic state), the Curie-Weiss law explains the temperature dependence of the susceptibility

$$
\chi=\frac{\mu_{0} N}{3 k_{B}} \frac{\mu_{\mathrm{eff}}^{2}}{T-\theta_{p}}
$$

and can be used to determine the effective magnetic moment, $\mu_{\mathrm{eff}}$, and the divergence temperature, $\theta_{p}$, which is usually close to the ferromagnetic (Curie) ordering temperature, $T_{C}$. In the ReNs the magnetic moment originates primarily from the unfilled Re $4 f$ shell. As described in Section 3.3, the spin and orbital moments of the electrons in the $4 f$ shell cannot be considered independently and must be coupled together to form a multiplet state. In LS coupling the ground state multiplet is determined by Hund's rules. Table 3.2 shows the calculated energetic separation between the ground state and first excited multiplets for the $\mathrm{Re}^{3+}$ ions. The separation is more than $100 \mathrm{meV}$ $(\sim 1000 \mathrm{~K})$, too large for the excited multiplet to be thermally populated. However, an applied magnetic field can induce an admixture of the multiplets. In this case the susceptibility is best explained by a modification of 
the Curie-Weis equation, the Van Vleck equation

$$
\chi=\frac{\mu_{0} N}{3 k_{B}}\left[\frac{60 \mu_{B}^{2}}{7 \Delta E}+\frac{\mu_{\mathrm{eff}}^{2}}{T-\Theta_{p}}\right],
$$

where $\Delta E$ is the energy difference between the two lowest energy multiplets $[59,60]$. This approach is used for the analysis of the $\mathrm{SmN}$ susceptibility data in Section 7.2.1.

Below $T_{C}$, a field dependent magnetization curve will show hysteresis. From a hysteresis loop the maximum magnetization (saturation moment), sample magnetization after removing a large applied field (remanence), and the field required to return a sample to a zero magnetization state (coercive field), can all be trivially extracted.

Measuring the temperature dependence of the magnetization as a function of temperature allows for a measurement of $T_{C}$. Typically a sample is prepared by cooling with, and without, the presence of a small applied field and then measuring the magnetization as the sample is heated in a small applied field. These techniques are referred to as field- and zero-field-cooled (FC and ZFC) measurements, respectively. Without an applied field to align its magnetic domains, a sample will order to minimize the total field, thus the ZFC magnetization will be small at temperatures well below $T_{C}$, so long as the applied field is less than the coercive field. In that sense comparing the FC and ZFC magnetization provides a quick hysteresis measurement. As the temperature increases toward the phase transition, the coercive field will decrease and the ZFC magnetization will increase until it is equal to the FC magnetization. The temperature at which this first occurs is approximately $T_{C}$.

The response of ReN thin films to applied magnetic fields was measured using a Quantum Design magnetic property measurement system (MPMS) superconducting quantum interference device (SQUID). Measurements were performed from $2 \mathrm{~K}$ to $300 \mathrm{~K}$, in fields up to $6 \mathrm{~T}$. ReN films grown on either Si or YSZ, and capped with GaN or YSZ, were cleaved to a size of roughly $2 \mathrm{~mm} \times 5 \mathrm{~mm}$ and inserted into a plastic sample holder that has no 
magnetic response. Data presented in this thesis were obtained and analyzed by S. Granville, C. Meyer, and B. Ludbrook.

\subsection{Optical Transmission}

Optical spectroscopy is the use of visible or near-visible light to interrogate a material. In principle a combination of reflectance and transmittance measurements, or ellipsometry, can be used to obtain information about the energy dependence of the complex index of refraction of a material, which depends heavily on direct interband transitions and can be directly related to numerical calculations of optical conductivity (see e.g., Ref. [61]). In practice the cap/film/substrate configuration required for any ex situ experiment on the ReNs complicates the extraction of the refractive index. With a well characterized capping layer, and well determined film thicknesses, it is still theoretically possible to extract the refractive index through a fitting of the Fresnel equations generalized for multiple interfaces, but small experimental uncertainties in any of the input parameters can lead to huge uncertainties in the refractive index. Regardless, an absorption edge can still be extracted from optical transmittance data. This slightly less quantitative approach provides valuable information since the absorption edge provides a measurement of the size of the direct band gap, a parameter that is essential for tuning numerical band structure calculations.

Optical transmittance data were obtained for GdN and SmN by Fourier transform infrared spectroscopy (FTIR) and for DyN by conventional transmittance techniques. For GdN the temperature of the sample was varied so that measurements were made above and below the ferromagnetic transition temperature. In transmittance mode FTIR measures the total transmitted intensity of broadband light as the path length of the photon is modulated as a function of time. The Fourier transform of this time dependent transmission determines the frequency (energy) dependent transmittance. Spectra were obtained over 0.2-2.0 eV with a Bomem model DA8 FTIR spectrometer using 
films grown on sapphire and YSZ substrates. For variable-temperature measurements the film was mounted in a flow-through cryostat with polyethylene windows. The conventional transmittance spectra were obtained from a monochromatized broadband light source over 1.1-3.2 eV. In both experiments the transmitted intensity was normalized by dividing by the incident intensity, measured simply by removing the sample.

\subsection{X-ray Spectroscopy}

Synchrotron based x-ray spectroscopy techniques can be used to obtain a wealth of data aboute the electronic structure of materials that is not obtainable with any other technique. The synchrotron itself, from this perspective, can simply be considered a source of extremely bright light with photon energies that range from the far infrared to hard x-ray. The radiation emitted from a modern synchrotron is generated by one of three radiation sources: a bending magnet, a wiggler, or an undulator. Each of these accelerate an electron with a magnetic field to produce radiation, but the output characteristics are quite different. Connected to the synchrotron are usually a number of different beamlines. The monochromators and measurement equipment at each beamline will vary depending on experimental goals.

The soft x-ray spectroscopy detailed in this thesis was performed on beamline X1B at the National Synchrotron Light Source at Brookhaven National Laboratory, and on beamline 511 at MAXlab in Lund, Sweden. Both X1B and 511 are undulator beamlines. The undulator generates a radiation spectrum that peaks strongly at a particular energy, the energy of which is determined by the spacing of the undulators magnetic poles. While both beamlines are equipped with a variable spacing undulator, the MAXlab 511 undulator is computer controlled and the spacing of the poles can be varied automatically from $23 \mathrm{~mm}$ up to $250 \mathrm{~mm}$, allowing measurements at any energy to be made on the undulator peak [62]. Both beamlines are fitted with spherical grating monochromators, and their end stations include the ability to 
perform x-ray absorption and x-ray emission spectroscopy. The Brookhaven X1B beamline measures x-ray absorption by total electron yield. At MAXlab 511 the total fluorescent yield technique is used. Both end stations are fitted with a Nordgren-type grazing-incidence soft x-ray emission spectrometer.

In this thesis x-ray absorption spectroscopy (XAS), ${ }^{4} \mathrm{x}$-ray emission spectroscopy (XES), and the closely related technique of resonant x-ray emission spectroscopy (RXES) were all used to extract information about the ReN electronic structure. XAS, XES and RXES define a class of techniques we refer to as XXS.

\subsubsection{X-ray absorption}

In XAS the fraction of light absorbed by a material is measured as a function of energy. The fundamental principles and techniques are described in Ref. [63]. In general the absorption is described by the Lambert-Beer law

$$
I(E, d)=I_{0} e^{-\mu(E) d}
$$

where $I_{0}$ is the intensity of the monochromatic incident beam and $I$ is the intensity after the beam passes through a material of thickness, $d$. The absorption coefficient, $\mu$, is energy and material dependent, but typical penetration depths in the soft x-ray energy band (roughly $120 \mathrm{eV}$ to $1200 \mathrm{eV}$ ) are $10 \mathrm{~nm}$ or more $[64,65]$.

In general the absorption coefficient decreases as $E^{-3}$, however, when the photon energy is close to the binding energy of a core level there is a large jump in the absorption coefficient [66]. This is referred to as an absorption edge. The different core states in an atom are commonly labeled according to their principle quantum number and their spin-orbit split core levels, the two factors which primarily affect their binding energy. The nomenclature

\footnotetext{
${ }^{4}$ Sometimes referred to as near edge x-ray absorption fine structure (NEXAFS) or xray absorption near edge structure (XANES), in order to distinguish from extended x-ray absorption fine structure (EXAFS), which is used to extract information about the local crystal structure rather than the local electronic structure.
} 


\begin{tabular}{|l|l|l|l|}
\hline Edge & Atom & $\mathrm{N}(\mathrm{eV})$ & $\mathrm{Gd}(\mathrm{eV})$ \\
\hline $\mathrm{K}$ & $1 \mathrm{~s}$ & 409.9 & 50,239 \\
$\mathrm{~L}_{1}$ & $2 \mathrm{~s}$ & 37.3 & 8,376 \\
$\mathrm{~L}_{2,3}$ & $2 \mathrm{p}_{\frac{1}{2}, \frac{3}{2}}$ & & 7,$930 ; 7,243$ \\
$\mathrm{M}_{1}$ & $3 \mathrm{~s}$ & & 1,881 \\
$\mathrm{M}_{2,3}$ & $3 \mathrm{p}_{\frac{1}{2}, \frac{3}{2}}$ & & 1,$688 ; 1,544$ \\
$\mathrm{M}_{4,5}$ & $3 \mathrm{~d}_{\frac{3}{2}, \frac{5}{2}}$ & & 1,$222 ; 1,190$ \\
$\mathrm{~N}_{1}$ & $4 \mathrm{~s}$ & & 379 \\
$\mathrm{~N}_{2,3}$ & $4 \mathrm{p}_{\frac{1}{2}, \frac{3}{2}}$ & & $286 ; 271$ \\
$\mathrm{~N}_{4,5}$ & $4 \mathrm{~d}_{\frac{3}{2}, \frac{5}{2}}$ & & $; 143$ \\
$\vdots$ & $\vdots$ & & $\vdots$ \\
\hline
\end{tabular}

Table 4.1: Comparison of terms used to describe core levels in x-ray spectroscopy and atomic physics. Relevant binding energies for $\mathrm{N}$ and $\mathrm{Gd}$ are shown. Values are from Ref. [66].

and typical electron binding energies for $\mathrm{N}$ and Gd are detailed in Table 4.1. The XXS in this thesis was measured at either the N K-edge or at the Re $\mathrm{M}_{4,5}$-edges (which we now refer to as the M-edge).

The absorption coefficient depends on the probability of transition of an electron from a core level to unoccupied states in the conduction band (CB) and can be calculated from the Fermi golden rule [63]

$$
P_{f i}=\frac{2 \pi}{\hbar}\left|\left\langle f\left|T_{1}\right| i\right\rangle\right|^{2} \zeta_{f}(E) .
$$

Here, the transition probability, $P_{f i}$, from an initial state, $|i\rangle$, to a final state, $|f\rangle$, depends on the density of final states, $\zeta_{f}(E)$, and the dipole operator, $T_{1}$.

In the soft x-ray region the dipole approximation is valid and gives rise to the dipole selection rule - that the difference in the Azimuthal (orbital) quantum number between the initial and final states is specified by $l_{f}-$ $l_{i}=\Delta l= \pm 1 .^{5}$ Thus the final state for XAS at the $\mathrm{N}$ K-edge will involve

\footnotetext{
${ }^{5}$ For multiplets, where the spin and orbital moments cannot be considered indepen-
} 
conduction band states that have $\mathrm{N} p$ character, and so N K-edge XAS measures the $\mathrm{N} p$-projected PDOS. XAS at the Re M-edge will involve final states that have Re $p$ or $f$ character. For the rare-earths the M-edge XAS is dominated by transitions to $4 f$ levels, although it does not directly measure the $f$-projected PDOS since multiplet effects are important.

In principle XAS can be measured in transmission mode by determining the ratio of the incident and transmitted x-ray intensities. However, for our thin film samples the substrate renders this technique useless and indirect methods are used. In our experiments the absorption was measured in total electron yield (TEY) and total fluorescent yield (TFY) modes and normalized to the incident beam intensity using standard techniques [63]. The excited state generated by XAS can decay via several channels. Typically, the relaxation is dominated by Auger or Coster-Kronig processes which result in the emission of large numbers of low energy electrons from the sample. The number of electrons produced is proportional to the x-ray absorption, allowing it to be measured practically. This is the TEY technique. It is also possible for the electron relaxation to excite a photon from the sample. Again, the flux of the x-rays emitted from the sample is proportional to the absorption. Measuring the radiation by a channeltron electron multiplier sensitive to x-rays allows the XAS to be measured by TFY [67]. In each case the incident beam passes through a gold mesh which is also measured by TEY. This allows the XAS to be corrected for the intensity of the incident beam.

In the energy ranges of typical soft x-ray spectroscopy, photo-emitted electrons generated in the TEY process generally originate from the surface layer. On the other hand the emitted x-rays measured by TFY can be emitted as far down as the x-ray penetration depth. This means that in a TFY measurement most of the XAS signal originates from the bulk of the sample so surface effects are less important. An added advantage is that since XAS is atom specific, thin capping layers can be used so long as the capping layer

dently, the dipole selection rule is expressed as $\Delta J= \pm 1$ and also $\Delta J=0$, if $L \neq 0$. 
does not contain the atom being interrogated.

XAS creates a core-hole that affects the potential of the atom so that the resulting electronic structure is different to the ground state. In fact, analysis of the dynamical many-body effects in model systems suggests that the correct potential to use for the absorption includes the core-hole on the absorbing atom, while for emission the correct potential is that without the core-hole $[68,69]$. This is known as the "final-state rule". Other investigations have found that the best description is achieved somewhere between the initial- and final-state rules and have suggested that it depends on whether the system is metallic or insulating [70].

\subsubsection{X-ray emission}

As noted above, it is most likely for the core-hole created by XAS to be filled by an electron cascade. However, there is a small probability (less than $1 \%$ at the K-edge) that it is instead filled by a direct transition from a valence band state. These transitions result in the emission of an x-ray, which can be measured. The development of the Nordgren-type grazing incidence spectrometer revolutionized the field by making it practical to obtain an energy resolved map of the emission intensity over the entire soft x-ray range [65, 71].

XES has the same advantage as TFY XAS, namely that X-rays can penetrate the bulk, and can be measured through a thin capping layer. The key disadvantage in XES is a direct result of the low XES yield. A long integration time, often up to an hour, is required to obtain a spectrum with a high enough signal to noise ratio. There are few sources of external noise in the soft x-ray regime, meaning that long integration times are generally not too problematic. Even then, typical energy resolutions are at best $200 \mathrm{meV}$ [65], significantly worse than the resolutions achieved in x-ray photoemission spectroscopy.

The dipole selection rule (see above) also applies in XES and so N Kedge XES measures the N p-projected PDOS of the occupied states. Thus, a combination of $\mathrm{N}$ K-edge XES and XAS allows the direct measurement of 
the N p-projected PDOS occupied and unoccupied states.

\subsubsection{Resonant x-ray emission}

In XES the core-hole is created by using a photon energy much higher than the absorption edge, essentially ionizing the atom. The resulting XES is considered a separate event and is non-resonant. However, if the core electron is excited into an unoccupied band state close to the Fermi level, then some fraction of the XES is resonant (RXES) and cannot be considered as a separate process to the excitation. Instead the whole process is described as a scattering event. The Kramers-Heisenberg formula describes both the first order XES and second order RXES [72], where the RXES scattering cross-section is given by [73]

$$
\frac{d \sigma}{d \Omega} \propto\left|\sum_{m} \frac{\left\langle f\left|T_{1}\right| m\right\rangle\left\langle m\left|T_{1}\right| i\right\rangle}{E_{m}-\hbar \omega-i \Gamma_{m} / 2}\right|^{2} \delta\left(E_{f}+\hbar \omega^{\prime}-E_{i}-\hbar \omega\right) .
$$

Here, the incident and emitted photon energies are $\hbar \omega$ and $\hbar \omega^{\prime}$, respectively. The initial (ground) state, intermediate (core-hole, electron in CB) state, and final (hole in VB, electron in CB) states are labelled as $|i\rangle,|m\rangle$ and $|f\rangle$, respectively. The energies of the various electron states are given by $E_{x}$.

In this picture the final state differs from the initial state only in an electron-hole pair that is present in the $\mathrm{VB} / \mathrm{CB}$. If the photon momentum is assumed to be negligible, conservation of momentum implies that the electron and hole must have the same crystal momentum - the resonant part of the RXES is $\mathbf{k}$-selective. Thus, while XAS and XES measure the PDOS across the entire Brillouin zone, RXES can in principle be used to obtain information about band dispersion. The nature of the resonant process means that this holds only for weakly correlated systems and excitation energies close to the absorption edge, where phonon and impurity scattering, which reduce the resonant effect, are minimal.

RXES, as measured, contains both incoherent XES and a k-conserving coherent contribution. In principle a full Kramers-Heisenberg treatment us- 
ing using the calculated band structures will produce spectra that reproduce the experimental signal. In practice this is difficult to implement. Instead it is customary to extract the coherent fraction (CRXES) by subtracting as much of the XES from the RXES as possible, while ensuring that the resulting spectrum is not at all negative.

In conjunction with XAS and a calculated band structure it is possible to determine which conduction bands and which portion of the Brillouin zone the incident photon excites core electrons to. The CRXES will be emitted from valence bands in the same portion of the Brillouin zone. This is the general recipe used to compare CRXES to band structure calculations in this thesis.

\subsubsection{X-ray photoemission spectroscopy}

X-ray photoemission spectroscopy (XPS) is another technique that can be used to probe the occupied states of a crystalline material. XPS is essentially the photoelectric effect in action and differs from XAS TEY at a fixed x-ray energy only in that the kinetic energy of the emitted electron is resolved. Using the simple relation between the energy of the emitted electron, $E$, the incident photon energy, $\hbar \omega$, and the electron work function, $\phi$,

$$
E=\hbar \omega-E_{k}-\phi
$$

it is possible to determine the binding energy, $E_{k}$, of the electron in the sample. Since dipole selection rules do not affect photoemission, XPS measures the total density of states (DOS) relative to the Fermi level of a sample (as opposed to the PDOS measured in XXS). The ability to measure the DOS gives XPS a significant advantage over XES when attempting to locate the energy of occupied $4 f$ levels.

In angle resolved photoemission spectroscopy (ARPES) the momentum of the photo-electron is resolved, as well as its energy. This is achieved by measuring the angle of the emitted electron relative to the sample surface, which can be related to the crystal momentum. This allows a measurement of 


\begin{tabular}{|c|c|c|c|c|}
\hline Material & Lab & \multicolumn{2}{|c|}{ XAS (eV) } & XES (eV) \\
\hline & & K-edge & M-edge & K-edge \\
\hline SmN & B & 0.2 & 0.4 & 0.4 \\
EuN & B & - & 0.4 & - \\
GdN & M & 0.2 & 0.4 & 0.2 \\
DyN & B & 0.2 & - & 0.4 \\
LuN & M & 0.2 & - & 0.3 \\
HfN & M & 0.2 & - & 0.4 \\
ZnO & B & 0.2 & - & 0.37 \\
\hline
\end{tabular}

Table 4.2: The experimental resolution of the various XXS measurements. Measurements were performed at either Brookhaven (B) or MAXlab (M).

the energy and $k$-resolved DOS, allowing for direct comparison with electronic band structure calculations. Experimental resolution is extremely good, with energies resolved down to the order of $10 \mathrm{meV}$. The techniques of XPS and ARPES are detailed in Ref. [74].

However, photoemission spectroscopy has a number of difficulties associated with it. The trajectory of an electron is modified by magnetic fields, meaning measurements of magnetic samples are difficult. Also, because it is a photoemission technique, the measurements are surface sensitive, insulating samples are susceptible to charging, and capping layers cannot be used. For these reasons XES and RXES are extremely useful, and sometimes superior, complimentary techniques.

\subsubsection{Experimental details}

The energy resolutions of the XXS presented in this thesis are shown in Table 4.2. 


\section{Chapter 5}

\section{Growth and Basic Characterization}

The problem of determining the electronic structure of a material through experiment is intractable without a source of high-quality, well-characterized samples. For the ReNs this is an open problem - there are few reports of the growth of the materials in the literature, in bulk or thin film form, and fewer still that are not plagued by the propensity of the ReNs to oxidize $[12,15$, 38]. Research presented in this thesis has focused on two relatively similar techniques for thin film growth: thermal evaporation deposition (TED), and pulsed laser deposition (PLD).

The choice of growth technique is only one of many free parameters that need to be tuned in order to optimize thin film growth. For example choice of substrate, growth temperature, and nitrogen partial pressure are all very important for producing a good ReN film. Also, since the ReNs react readily with ambient atmosphere, any ex situ experiment requires a passivating capping layer. This added constraint means any intended experiment must also be considered when choosing substrate and capping layers. With these considerations in mind many films have been produced and the best chosen for this study.

Structural characterization provides essential feedback for tuning the pa- 
rameters involved in film growth. Reflection high-energy electron diffraction (RHEED) and x-ray diffraction (XRD) were both used extensively to determine the crystalline nature of the ReNs, and a combination of Rutherford backscattering spectroscopy (RBS) and nuclear reaction analysis (NRA) was used to determine the film stoichiometry to within $\sim 1 \%$.

The vast majority of work on film optimization has focused on GdN and SmN but, due to the chemical similarity of the ReNs, the following results are expected to generalize reasonably well across the series. Indeed this has been demonstrated in a limited number of growths of DyN, ErN, and LuN. The results show that it is possible to produce ReN thin films with highly reproducable characteristics and variations that can be controlled by choice of growth parameters. Significant progress has been made toward the end goal of epitaxial single crystal thin films. TED films are found to be stoichiometric within measurement uncertainties and polycrystalline with crystallites of roughly $10 \mathrm{~nm}$ in diameter while PLD films are found to grow epitaxially with large crystallites of roughly $30 \mathrm{~nm}$ and are also stoichiometric.

\subsection{Film Deposition Techniques}

In binary alloys, like the ReNs, the art of thin film growth includes not just finding the optimal method for depositing a homogenous epitaxial layer on a substrate, but also tuning a number of parameters to encourage the formation of a high purity compound in the required stoichiometry. Typical nitride thin film growth techniques can be classified into three basic categories defined by either the reaction of a metal with possibly excited nitrogen gas (TED, $\mathrm{PLD}, \mathrm{MBE}^{1}$ ); or reactions between sources of often volatile precursors $\left(\mathrm{CVD}^{2}, \mathrm{MOCVD}^{3}\right)$; or, simply, the ablation or evaporation of powders of the

\footnotetext{
${ }^{1}$ Molecular beam epitaxy

${ }^{2}$ Chemical vapour deposition

${ }^{3}$ Metalorganic CVD
} 
desired end material onto a substrate (TED, PLD). In all cases care must be taken to minimize the inclusion of impurities, particularly oxygen. Usually an ultra-high vacuum (UHV) environment is necessary.

Detailed investigations into the growth of thin ReN films have only recently begun to appear in the literature. Largely they have examined GdN. Leuenberger et al. reported growth of thin films of polycrystalline GdN, although with more of a focus on electronic structure investigations than growth optimization [39, 41]. More recently Gerlach et al. presented evidence for epitaxial growth on $\mathrm{MgO}$ (001) by MBE [75], and a very recent report has shown GdN (111) epitaxy on GaN (0001), also by MBE [76].

Below, we detail two growth methods based on the reaction of raw metal with nitrogen - TED and PLD - and examine the key parameters that affect film quality. For both growth techniques the target film thickness was generally $200 \mathrm{~nm}$.

\subsubsection{Thermal evaporation deposition}

TED is a UHV technique based on the evaporation of rare-earth metal in the presence of $\mathrm{N}_{2}$ gas. To grow a film the UHV chamber is first pumped to a base pressure of less than $10^{-8}$ mbar. The metal is then evaporated using either an electron gun or thermal evaporator, and ultra-high purity $\mathrm{N}_{2}$ gas $(6 \mathrm{~N})$ is flowed through the system at a rate designed to mantain a partial pressure of between $10^{-5}$ and $10^{-4}$ mbar. To further scrub the gas of potential oxygen or water contaminants it is passed through an Aeronex Ni sponge filter. The ReN growth rate, monitored by a piezoelectric quartz crystal monitor, is maintained at a constant $1 \AA^{-1}$ by a deposition rate controller. For all of the TED samples described in this thesis no attempt was made to excite the nitrogen gas, or heat the substrates, during the deposition process. Details of the growth technique are found in the thesis by S. Granville [77] and in Ref. [78]. The TED system has been used to grow polycrystalline thin films of SmN, GdN, DyN, ErN, and LuN. 


\subsubsection{Pulsed laser deposition}

The PLD was done using the system maintained by S. Durbin et al. of Canterbury University. The system is based around a Lambda Physik Compex $205 \mathrm{KrF}$ excimer laser operating at a wavelength of $248 \mathrm{~nm}$. In general the laser was pulsed at $10 \mathrm{~Hz}$ with a pulse length of $25 \mathrm{~ns}$ and a fluence of roughly $5 \mathrm{Jcm}^{-2}$. The laser was used to ablate a $99.9 \%$ pure rare-earth target in the presence of ultra-high purity nitrogen gas $(5 \mathrm{~N})$, maintained at pressures in the same range as the TED system. The PLD technique is very similar to TED in that the film is produced from a rare-earth metal source evaporated in the presence of $\mathrm{N}_{2}$ gas.

The PLD system also has some significant advantages over the TED system used in this study. In particular a RHEED system allows monitoring of the in-plane crystal ordering and lattice constant during growth. Also, an Oxford Applied Research RF plasma source, used to generate excited nitrogen species, is attached to the chamber. It was operated at powers ranging from 120-400 W, before an optimum power of $200 \mathrm{~W}$ was determined. Further, the substrate was held at temperatures ranging from $500-900^{\circ} \mathrm{C}$ during growth. These parameters were chosen based on prior experience with the growth of other nitrides, in particular GaN [79]. Additionally a load-lock allows fast sample turnaround.

On the other hand, the system is disadvantaged by the lack of a quartz crystal monitor. To determine approximate growth rates either rare-earth metal was ablated for a specific period of time and a thickness was obtained by etching and subsequent profilometry, or RBS was used. From these techniques an approximate growth rate of $1 \AA / \mathrm{s}$ was obtained. It is also noted that the pulsed nature of the evaporation technique means that the flux rate of the rare-earth at the substrate will not be constant and that large droplets of metal can be ablated and incorporated into samples. The PLD system has been used to grow SmN, GdN, LuN, and HfN. 


\subsubsection{Substrates}

For epitaxial growth of thin films it is important to have a compatible substrate. In the case of wurtzite materials, like $\mathrm{GaN}$, growth is generally achieved by depositing a low temperature buffer layer on a sapphire or $\mathrm{SiC}$ substrate [79]. Unlike most of the III-V semiconductors which bond covalently, the ReNs are largely ionic and crystallize in the rocksalt ( $\mathrm{NaCl}, \mathrm{Fm} \overline{3} \mathrm{~m})$ crystal structure. These differences mean that standard nitride growth techniques will not necessarily be directly transferable to the growth of the ReNs.

Growth on $\mathrm{Si}$ is desirable, since there is a large amount of interest in integrating spintronic devices with conventional Si based electronics. However, it is believed that silicide formation at the interface prevents epitaxial growth of GdN on silicon [80]. On the other hand a successful integration of EuO on Si has been reported [81].

Other cubic substrates have been considered. A recent report provided evidence for the epitaxial growth of $\mathrm{GdN}$ on $\mathrm{MgO}$, which is perhaps surprising considering the lattice mismatch of $+18.7 \%$ [75]. Another promising material is yttria stabilized zirconia (YSZ). Zirconia $\left(\mathrm{ZrO}_{2}\right)$ is stable in the monoclinic crystal structure at room temperature, but is found in the cubic crystal phase at high temperatures. This phase can be stabilized at room temperature by the addition of ytrria. YSZ has a lattice constant of $5.12 \AA$, meaning that lattice mismatches with the ReNs are at most $8 \%$ (for $\mathrm{LuN}$ ), and are much lower for the ReNs that have seen the most interest (the GdN lattice mismatch is less than $3 \%$ ). It is noted that both $\mathrm{MgO}$ and $\mathrm{YSZ}$ are oxides, and that oxygen contamination is an important consideration in the growth of nitrides. Indeed it has been shown that a YSZ substrate can cause significant oxygen contamination of GdN due to the high mobility of oxygen and nitrogen ions in YSZ, especially at temperatures above $600^{\circ} \mathrm{C}$ [82]. 


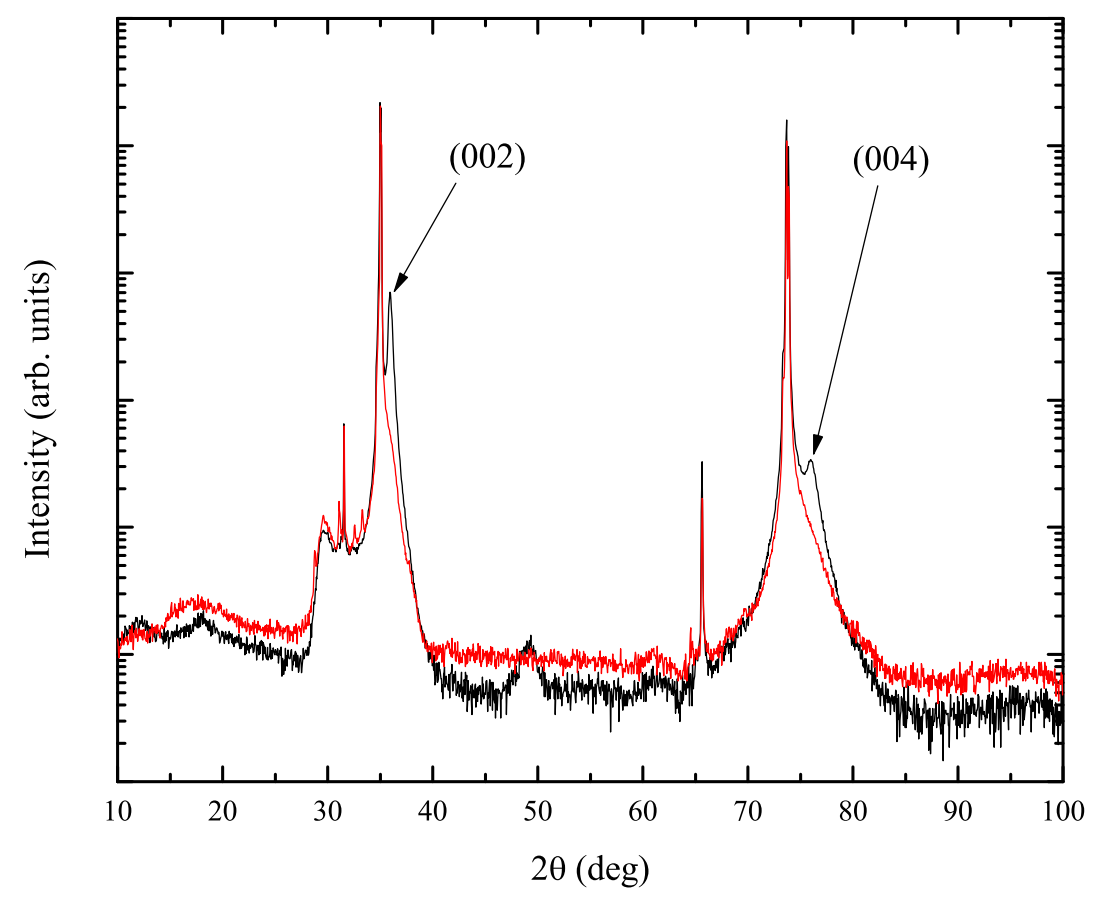

Figure 5.1: XRD spectra of GdN thin films grown on YSZ by PLD. One film is capped with YSZ (black line), while the other is not (red line). The GdN (00n) peaks are labelled.

\subsubsection{Capping layers}

The ReNs react readily with the water and oxygen that is present in the ambient atmosphere [15]. This means a passivating layer is essential if any ex situ studies are to be attempted. An informed choice of capping layer (and substrate) is important since it constrains the range of experiments that will be possible with that film. To measure all of resistivity, magnetism, and optical and x-ray spectra it is often necessary to produce a number of films with the same growth parameters but different capping layers.

In our experiments $\mathrm{GaN}, \mathrm{AlN}, \mathrm{Ag}, \mathrm{Cr}, \mathrm{MgF}_{2}$, and YSZ have all been used as capping layers. $\mathrm{Ag}, \mathrm{Cr}$, and $\mathrm{MgF}_{2}$ are simply evaporated, or ablated, from an appropriate source. In the TED system GaN and AlN are grown in a 
similar manner to the ReNs, except that ion assisted deposition is required. ${ }^{4}$ In the PLD system AlN and YSZ have been ablated from targets. Both $\mathrm{GaN}$ and YSZ increase the atmospheric lifetime of ReN films from minutes to weeks. Both have significantly larger bandgaps than the ReNs and can be grown with reasonable reliability. As a result they are used as the default capping layers in the TED and PLD systems, respectively.

In all cases ex situ XRD measurements are used to investigate the ReN crystal structure and the efficacy of the capping layer. Figure 5.1 shows the XRD spectra of two GdN films produced with identical growth parameters. In this case they were grown by PLD on a YSZ (001) substrate. For both films strong (00n) substrate peaks are seen at $35.0^{\circ}$ and $73.7^{\circ}$. One of the films was capped with YSZ, while the other was left uncapped. GdN has a lattice constant of $4.99 \AA$ which is slightly smaller than YSZ (5.12 $\AA)$, therefore the GdN (00n) peaks should be visible at a slightly higher angle than the equivalent substrate peak. In Fig. 5.1 the GdN peak is seen only in the capped film, highlighting the necessity of the capping layer.

The TED system allows the in situ measurement of the resistivity of a film, as detailed in Section 4.1. Consistency between the temperature dependent resistivity of films measured pre- and post-cap, and also ex situ, acts as a second confirmation that the measured properties are intrinsic to the film.

\subsection{Structural Characterization}

\subsubsection{Stoichiometry}

RBS and NRA indicate that the ReN films are stoichiometric, in the expected 1:1 ratio, to within the experimental resolution of approximately $1 \%$. This resolution is too low to make any meaninful statements about the nitrogen

\footnotetext{
${ }^{4}$ It is noteworthy that activated nitrogen is essential for the growth of III-V semiconductors, whereas the ReNs will grow in a passive nitrogen atmosphere.
} 
vacancy doping that is suspected to affect conduction. Thus, when we refer to stoichiometry measured by RBS/NRA, we are refering to the coarse stoichiometry of the film.

Hall effect measurements provide a more accurate estimation of the carrier concentration. Recent measurements show GdN films grown by PLD are electron donor doped to degeneracy, with carrier concentrations of $4 \times$ $10^{20} \mathrm{~cm}^{-3}[82]$. The donors are almost certainly nitrogen vacancies as shown by others [41], and by the relationship between GdN conductivity and nitrogen partial pressure that is observed during film growth [78].

Simply looking at a film can provide valuable information. A film generally starts to degrade when the capping layer develops pinholes which allow the atmosphere to attack the film. These are readily visible as they form. A microscope image of a film can also provide information on large scale inhomogeneities. Figure 5.4 (b) shows a microscope image of a GdN film grown by PLD. The dark areas are droplets of Gd metal with diameters on the order of microns. These droplets are only seen in PLD films and are common in films grown by ablation of a metal target. The implication is that while large areas of the substrate are covered by epitaxial GdN the metallic Gd phase must also be considered, especially in magnetization measurements.

\subsubsection{XRD}

Measured in the $\theta-2 \theta$ orientation, XRD provides information on the out-ofplane crystal ordering of a sample. The out-of-plane data supplements that obtained from RHEED (in Section 5.2.3), which provides information about the in-plane crystal ordering. Nomenclature is important when switching between these complimentary techniques. In this thesis the convention is that for a substrate cut to expose the (001) face the out-of-plane direction is [001] and the in-plane directions are [100] and [010]. Therefore peaks in the $\mathrm{XRD}$ are labelled as (00n) [59].

Figure 5.2 shows XRD obtained from each of the ReNs grown by TED and from GdN and HfN films grown by PLD. In each case more than one 


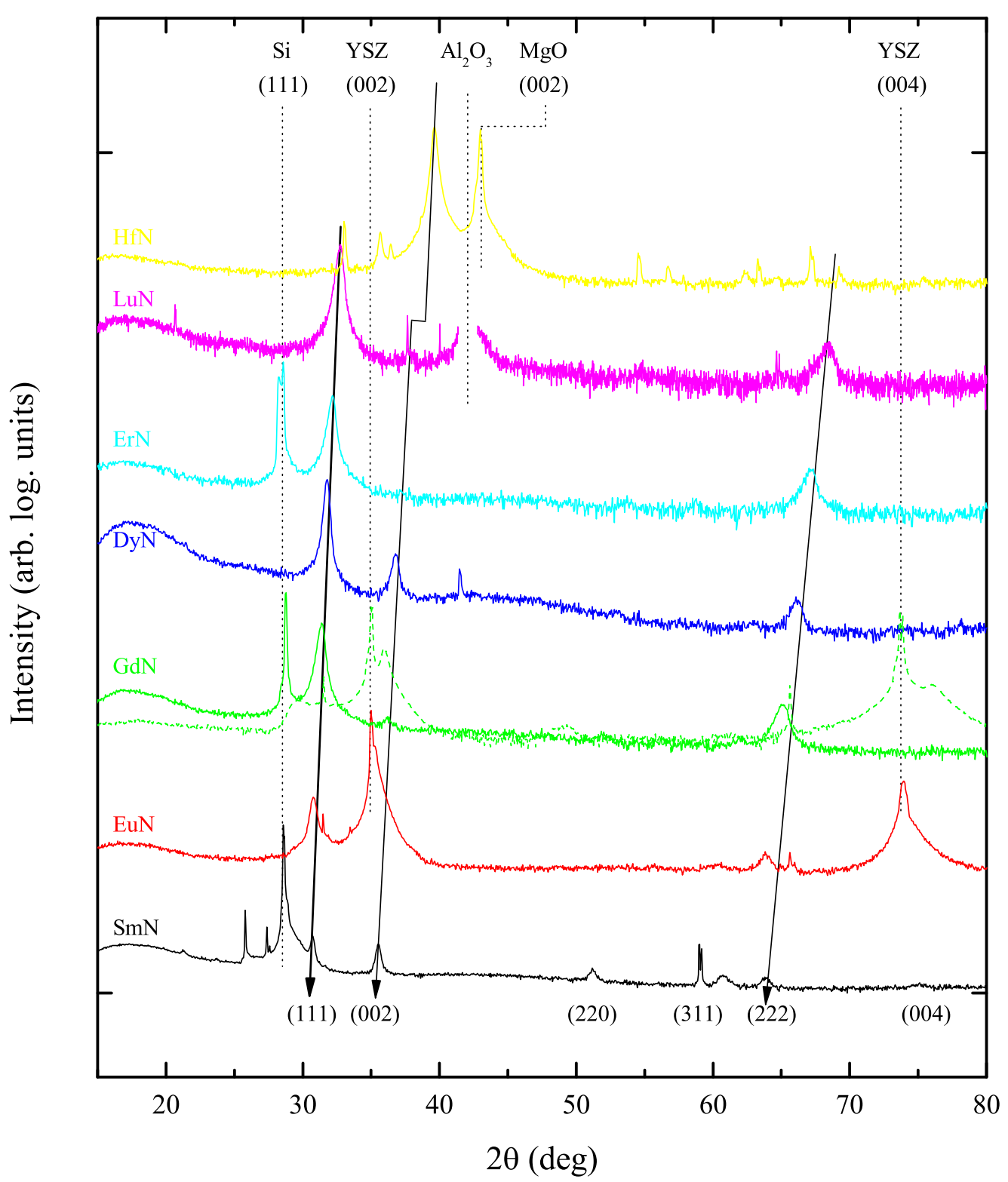

Figure 5.2: XRD spectra of ReN thin films. Top labels indicate substrate peaks, bottom labels indicate ReN FCC peaks. The lattice constants and average crystallite sizes extracted from these spectra are presented in Table 5.1 . 


\begin{tabular}{|c|c|c|c|c|c|}
\hline \multirow{2}{*}{ ReN } & \multicolumn{3}{|c|}{$\mathrm{a}(\AA)$} & \multicolumn{2}{c|}{$\mathrm{D}(\mathrm{nm})$} \\
\cline { 2 - 6 } & Expt & $(\mathrm{Pub})$ & Theory & $(002)$ & $(111)$ \\
\hline $\mathrm{CeN}$ & & $(4.87)$ & 4.90 & & \\
$\mathrm{PrN}$ & & $(5.17)$ & 5.29 & & \\
$\mathrm{NdN}$ & & $(5.15)$ & 5.24 & & \\
$\mathrm{PmN}$ & & & 5.19 & & \\
$\mathrm{SmN}$ & 5.050 & $(5.04)$ & 5.10 & 25.7 & 27.9 \\
$\mathrm{EuN}$ & 5.030 & $(5.00)$ & 5.14 & - & 19.0 \\
& 4.963 & $(4.98)$ & 5.08 & 16.1 & 16.4 \\
$\mathrm{GdN}$ & $4.990^{a}$ & & & 25.6 & - \\
$\mathrm{TbN}$ & & $(4.92)$ & 5.05 & & \\
$\mathrm{DyN}$ & 4.885 & $(4.90)$ & 5.08 & 15.5 & 18.2 \\
$\mathrm{HoN}$ & & $(4.87)$ & 4.98 & & \\
ErN & 4.818 & $(4.83)$ & 5.00 & - & 18.1 \\
TmN & & $(4.80)$ & 4.90 & & \\
YbN & & $(4.78)$ & 4.79 & & \\
LuN & 4.768 & $(4.76)$ & 4.87 & 9.1 & 15.0 \\
HfN & 4.544 & $(4.52)$ & 4.54 & 29.2 & 62.0 \\
\hline
\end{tabular}

Table 5.1: Lattice constant and average crystallite size of each of the ReN thin films in Fig. 5.2. Crystallite sizes were extracted from both (111) and (002) peaks. Experimental [15, 83, 84] and theoretical [13, 42] reference values for the lattice constant are also included.

${ }^{a}$ PLD sample. 
film has been produced; the spectra presented here are from films grown with what are determined to be optimal parameters for achieving stoichiometric film growth and large crystallite sizes. Table 5.1 shows the lattice constant extracted from the (002) peak of each spectrum except for EuN and ErN. In these two ReNs film growth is strongly textured along the (111) direction ${ }^{5}$ so the lattice constants are extracted from that peak instead. Also shown in Table 5.1 are the average crystallite sizes extracted from the (111) and (002) peaks. ${ }^{6}$ A value is omitted in cases where film is textured enough that the relevant peak is not present.

As evidenced by the spectra, and by the crystallite sizes, films grown by TED are either polycrystalline $(\mathrm{SmN}, \mathrm{GdN}, \mathrm{DyN}, \mathrm{LuN})$ or textured in the [111] direction (EuN, ErN). The difference between the GdN films grown by TED and PLD is striking. The TED film is polycrystalline while the PLD film is heavily textured.

The TED GdN film in Fig. 5.2 was grown on Si (111), the PLD film on YSZ (001). To eliminate the substrate as a variable, and to more closely examine the differences between TED and PLD, thin films of GdN were grown on YSZ (001) and a PLD film was deposited on YSZ (111). Figure 5.3 shows the XRD spectra. All films were capped with YSZ. A spectrum of a clean YSZ (001) substrate is also shown for reference. The film grown by TED on YSZ (001) shows a strong (111) peak and no sign of (00n) peaks,

\footnotetext{
${ }^{5}$ In other words the crystallites preferentially orient in the (111) direction. If the crystallites orient randomly the films would be powder like.

6 The lattice constant, a, was extracted from the XRD using Bragg's law

$$
\lambda=2 d \sin (\theta)
$$

where $\lambda=1.5418 \times 10^{-10} \mathrm{~m}$ is the wavelength of the $\mathrm{Cu} \mathrm{K}$ radiation used in the experiment. The average crystallite size, $\mathrm{D}$, was extracted using the Debye-Scherrer formula

$$
D=\frac{0.9 \times \lambda}{\beta \cos (\theta)}
$$

where $\beta$ is the full-width at half-maximum (FWHM, in units of $2 \theta$ ), and $\theta$ is the position of the peak in radians (i.e., half the $2 \theta$ position of the peak) [85].
} 


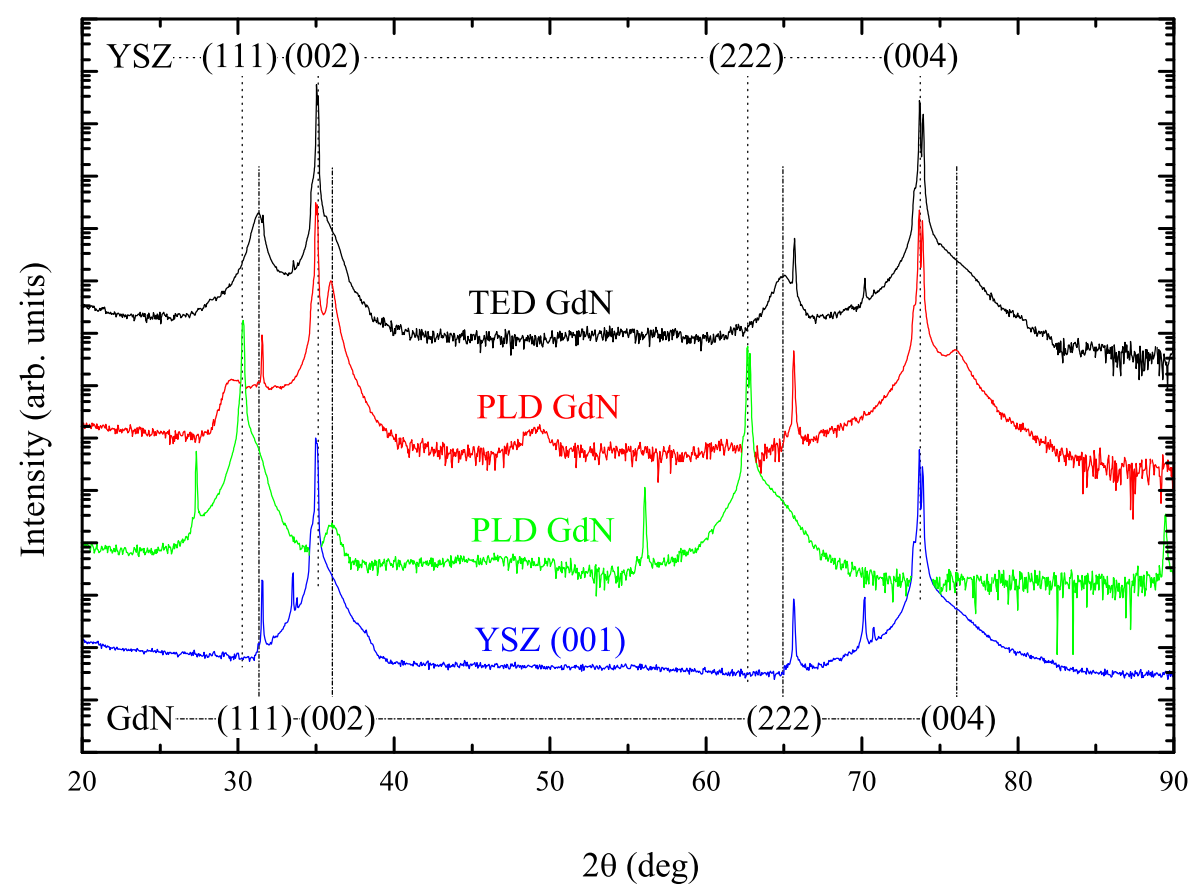

Figure 5.3: XRD spectra of GdN films grown on YSZ, and a reference YSZ scan. The bump at $49^{\circ}$ in the PLD GdN sample grown on YSZ (001) is thought to be due to the YSZ capping layer.

indicating that the texturing observed in the PLD growth is not due to the substrate alone, but also the growth technique. For the PLD films grown on both YSZ (001) and YSZ (111) the off-plane peak is heavily attenuated, providing evidence for epitaxial growth in the out-of-plane direction that is complemented by RHEED measurements of in-plane epitaxy.

\subsubsection{RHEED}

RHEED provides important information about the in-plane ordering of films during growth and allows for rapid fine tuning of growth parameters. A RHEED image, taken in the [100] direction near the end of the growth of a GdN film on YSZ (001), is shown in Figure 5.4 (a). The streaky lines indicate a high degree of in-plane coherence. This, together with XRD (Fig. 5.3), pro- 

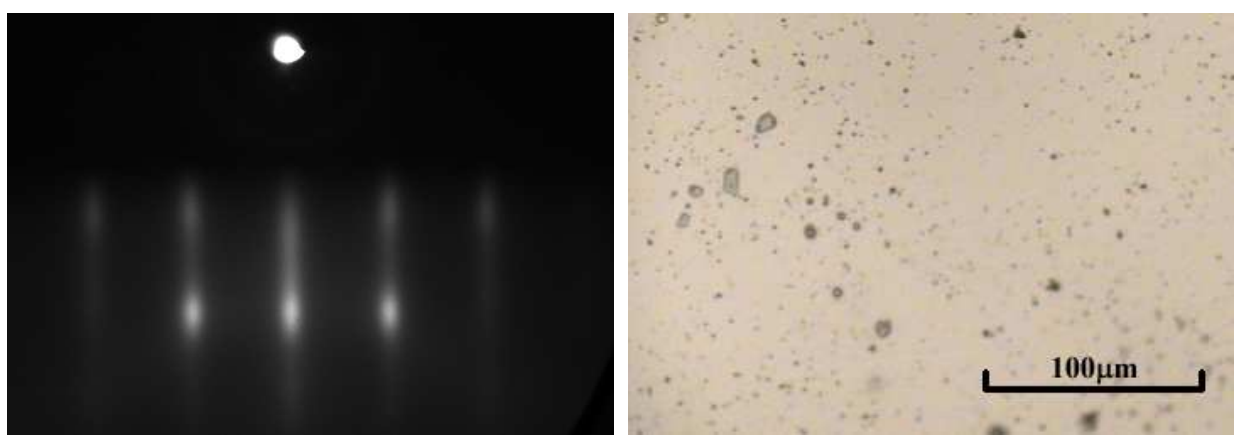

Figure 5.4: (a) RHEED image, and (b) microscope image of a GdN film grown by PLD. Streaks on the RHEED image indicate a high degree of inplane ordering. Small black dots on the microscope image are droplets of gadolinium metal.

vides strong evidence for the epitaxial growth of GdN by PLD. This evidence is detailed in the paper by Ludbrook et al. [82].

Given that the XRD spectra of films grown by PLD show much more evidence for epitaxy than those grown by TED, and that the RHEED confirms the epitaxial nature of the PLD films, it is important to understand which parameter in the PLD system most strongly affects crystal quality. A number of parameters were varied between growths and evaporation systems. In both systems the films were grown at a similar rate, with similar base pressures. Also, nitrogen partial pressures were varied in both systems. In the PLD system both substrate temperature and the power of the plasma source, which provides activated nitrogen species, were varied. The growth of both SmN and GdN was optimized by measuring RHEED quality as these parameters were varied. It has been determined that substrate temperature is the key driver of film quality, and that optimal growth temperatures are $500^{\circ} \mathrm{C}$ and $800^{\circ} \mathrm{C}$, for $\mathrm{SmN}$ and $\mathrm{GdN}$ respectively. 


\subsection{Considerations for X-ray Spectroscopy}

X-ray spectroscopy of the nitrogen K-edge is a key technique for characterizing the electronic band structure of nitrides and plays an important part in this thesis. To conduct $\mathrm{x}$-ray spectroscopy experiments it is necessary to get a suitable film into the UHV system at a synchrotron. Two methods of affecting this are considered: either grow a sample off-site and transport it to the facility, or grow a film in situ. The latter is an attractive option for the reactive ReNs because then capping layers do not need to be considered indeed, this is the technique used to obtain N K-edge spectra for SmN and DyN (Section 7.5). However film growth in the confines of a typical sample preparation chamber found at a synchrotron is difficult; it is harder to obtain the parameters required for optimal film growth. In the case of GdN the problem is complicated further by the high evaporation temperature of $\operatorname{Gd}\left(\sim 1250^{\circ} \mathrm{C}\right)$.

As a result it is desirable to find a capping layer that will allow a film to survive ambient conditions over the extended period of time required to transport it to the synchrotron, and that can either be removed once the film is under vacuum, or allows the measurement to be carried out through the capping layer. The requirement for N K-edge spectroscopy precludes the use of GaN, or any other nitride, as a capping layer, since the $\mathrm{N}$ in the capping layer will dominate the signal. Instead both $\mathrm{Cr}$ and YSZ are considered as capping layers.

$\mathrm{Cr}$ is desirable since it is electrically conducting, is relatively easy to sputter, and is considered x-ray neutral in the soft x-ray range. Its high conductivity means XPS can be used to monitor the thickness of the capping layer during sputtering, and that XAS using TEY should be possible through a thin layer. However, it was found that Cr layers as thick as $100 \mathrm{~nm}$ were required in order to protect the films for the requisite length of time, making it difficult to completely sputter the layer. Large sputter times are required to significantly thin a $100 \mathrm{~nm}$ layer, and after such a large sputter the surface will be very rough. Worst of all it appears Cr reacts with the film. Figure 5.5 


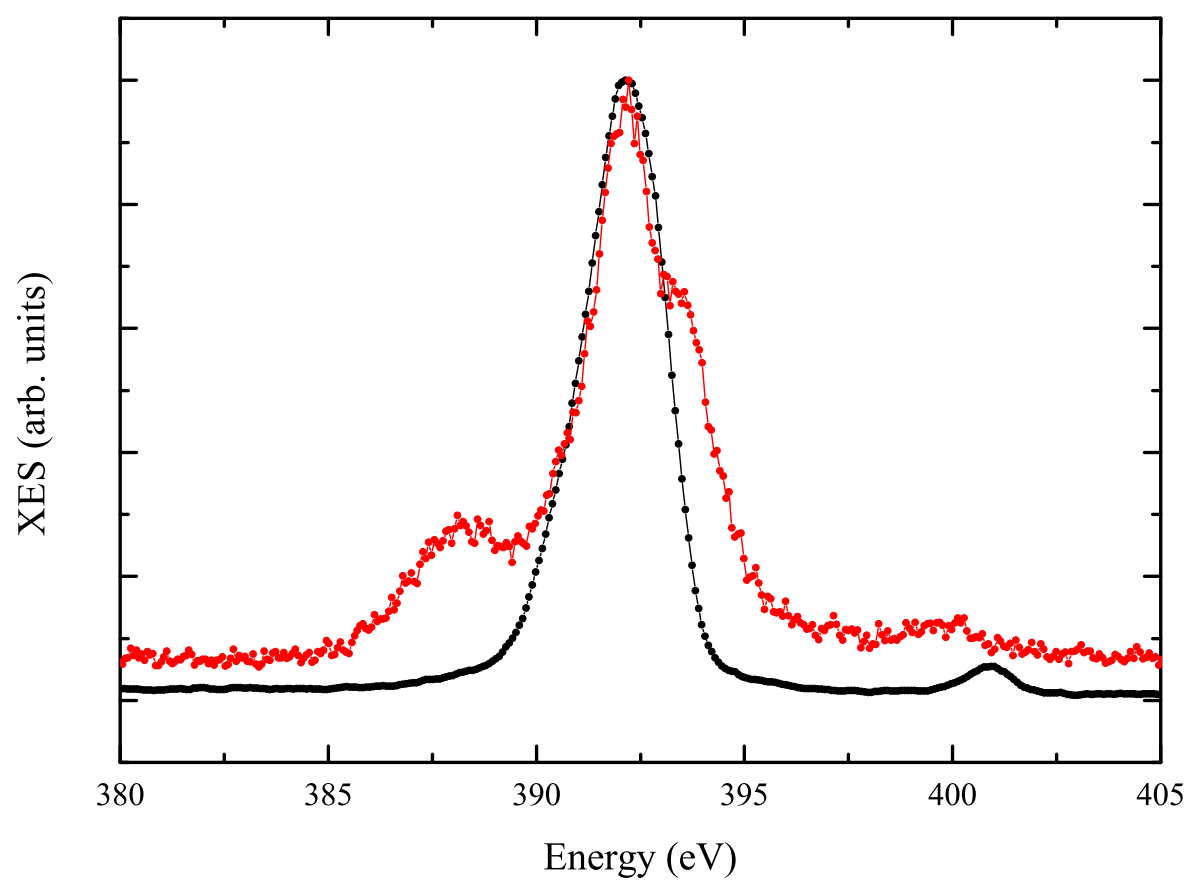

Figure 5.5: XES spectra of $\mathrm{SmN}$ grown in situ (black circles) and capped with $\mathrm{Cr}$ (red circles). An elastic peak is visible at $401 \mathrm{eV}$ in the in situ spectrum.

shows the XES spectra of a SmN sample grown in situ, and a sample grown by PLD and capped with Cr. The differences between the two spectra imply that the Cr significantly affects the signal, probably by forming a CrN layer at the interface. This finding may explain the differences between the GdN K-edge XAS reported by Leuenberger et al. [41] and the data presented in Section 6.7. A detailed study of the interaction of $\mathrm{Cr}$ capping layers with ReNs is necessary before they can be considered again as capping layers for N K-edge spectroscopy.

The insulating nature of YSZ means that any photoemission experiment will be difficult. However photon-in-photon-out techniques can be used. In this case both XES and XAS (using TFY or PFY) are possible, so long as the $\mathrm{x}$-ray penetration depth is larger than the capping layer thickness. Thin 
films of GdN and LuN, grown by PLD and capped with YSZ were successfully used to make XXS measurements. The droplets seen in PLD films should not significantly affect measurements since N K-edge spectroscopy measures the local $p$-projected PDOS around $\mathrm{N}$ sites; the majority of $\mathrm{N}$ sites are far away from Gd droplets.

\subsection{Conclusion}

Thin films of SmN, EuN, GdN, DyN, ErN, LuN, and HfN have all been produced using TED or PLD. RBS, NRA, and Hall effect experiments indicate that the films are stoichiometric and XRD shows that all films have the simple cubic FCC structure with expected lattice constants. RHEED studies of GdN grown by PLD provide evidence for epitaxial growth on YSZ substrates. YSZ was found to be a promising capping layer for measuring XXS by TFY, while $\mathrm{Cr}$ was proved to form a $\mathrm{CrN}$ interface which interferes with $\mathrm{N}$ K-edge spectroscopy. The films are judged to be of high enough quality to make significant progress with electronic structure investigations. 


\section{Chapter 6}

\section{GdN}

\subsection{Motivation}

GdN is the most studied of the ReNs, as evidenced by the literature $[12,13$, $38,41]$. GdN has the highest $\mathrm{T}_{C}$ of all the ReNs meaning that experimental investigations of the magnetically ordered ground state are easier, and potential applications more likely. Further, the absence of orbital moment in the ground state of the $\mathrm{Gd}^{3+}$ ion greatly simplifies magnetic characterization and band structure calculations where the main challenge lies in accurately incorporating the localized $4 f$ orbitals. Additionally, GdN has the the same crystal structure as $\mathrm{EuO}$ and both $\mathrm{Eu}^{2+}$ and $\mathrm{Gd}^{3+}$ share the same ${ }^{8} S_{7 / 2}$ ground state. Although $\mathrm{EuO}$ has received somewhat more attention than the GdN they both have very similar predicted and observed properties [86]. As a point of difference the GdN band structure is much more similar to conventional semiconductors. The GdN VBM consists of dispersive N $2 p$ states, while $\mathrm{EuO}$ has a flat VBM that is largely $4 f$ in character.

A number of theoretical studies have been devoted to $\mathrm{GdN}$ and the $\mathrm{Gd}$ pnictides. The nature of the ground state electronic and magnetic structure have both come under scrutiny. Calculations have at times labelled GdN as a half-metal $[36,37,87]$, although with experimental confirmation LSDA $+U$ and $G W$ calculations are converging on agreement that it is a semiconduc- 
tor $[13,53,88]$. The anti-ferromagnetic nature of the other Gd pnictides is well understood, with the large $\mathrm{T}_{C}$ of $\mathrm{GdN}$ now considered an important challenge to theory [89].

There are few modern experimental electronic structure investigations reported in the literature. Li et al. investigated the magnetic properties of GdN [31] and a recent report on MBE growth provided evidence of epitaxial growth, but did not describe the films electronic properties [75]. The most comprehensive investigation of the electronic structure thus far was reported by Leuenberger et al. [39, 41, 40]. They present resistivity, magnetization and x-ray spectroscopy data and observe striking changes in the magnetoresistance below $\mathrm{T}_{C}$, which is likely due to a shift in the electronic band structure caused by the phase transition. Still, a number of questions remain both about quality of previous reports and, more fundamentally, in the underpinnings of the electronic structure. For example, the size of the band-gap, the size of the exchange splitting, and the energetic location of the localized $4 f$ orbitals must all be elucidated in order to tune theoretical calculations.

Thin films of GdN have been grown by both TED and PLD. Their structural characterization is detailed in Chapter 5. In this chapter we probe their electronic structure. Temperature dependent resistivity, magnetization, and optical transmission are used to determine that GdN is a ferromagnetic semiconductor in the ground state and is also semiconducting above the ferromagnetic transition temperature of about $68 \mathrm{~K}$. The absorption edges obtained from optical transmission data are used to tune LSDA $+U$ based band structure calculations. XAS, RXES and x-ray magnetic circular dichroism (XMCD) measurements are all shown to be in strong agreement with the tuned theoretical predictions. The result is a comprehensive picture of the electronic structure of GdN. 


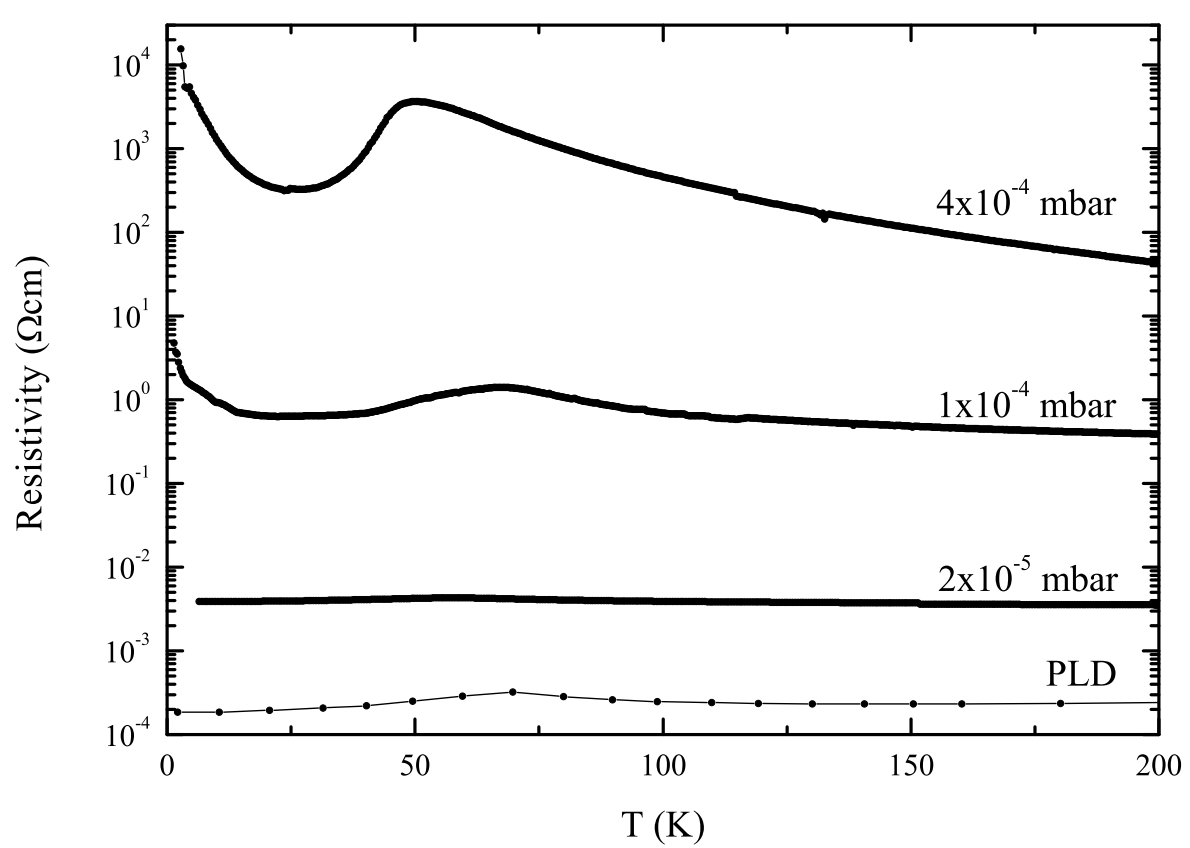

Figure 6.1: Resistivity as a function of temperature for representative GdN samples. In each case the peak in the resistivity coincides with the ferromagnetic ordering temperature. The label indicates the $\mathrm{N}_{2}$ partial pressure during TED growths.

\subsection{Transport}

Figure 6.1 shows the resistivity of selected GdN samples as a function of temperature. Three of the samples were grown by TED and one by PLD. The variation between the TED films was obtained by using different nitrogen partial pressures during growth. The film with the highest resistivity was grown in a nitrogen partial pressure of about $4 \times 10^{-4}$ mbar compared to $2 \times 10^{-5}$ mbar for the TED film with the lowest resistivity. The sample with the highest resistance is quite clearly semiconducting, while the sample with the lowest resistance - the PLD sample - almost appears to be metallic in that it has a resistivity of roughly $2 \times 10^{-4} \Omega \mathrm{cm}$. However, even for this sample, the absolute size of the ambient temperature resistivity is too large 
for a material that is intrinsically metallic, or semimetallic [90]. Instead the sample is likely a semiconductor that has been doped to degeneracy. The heavy doping is almost certainly a result of nitrogen vacancies as proved by the inverse dependence of sample conductivity on growth pressure, and also recent Hall effect measurements $[41,78,82]$. The rather large change in the resistivity at the ordering temperature of the PLD sample, relative to the TED sample with the most similar resistivity, is not well understood and will require further investigation.

Considering the temperature dependence from $75-300 \mathrm{~K}$, the resistivity of the TED samples decreases in a manner typical of a semiconductor while the positive temperature coefficient of resistance seen in the PLD sample is typical of a degenerately doped semiconductor. For the three samples that show a semiconducting behaviour any activation energy obtained from an Arrhenius type fit of the data is small (on the order of $10 \mathrm{meV}$ for even the highest resistivity sample), many orders of magnitude smaller than the calculated gap, indicating that even the sample with the highest resistivity is still heavily doped.

All samples show a peak in the resistivity at a temperature between 50 and $70 \mathrm{~K}$. This effect, attributed to changes in the band structure as the material transitions to the ferromagnetic ground state (see Section 6.4), was observed in GdN by Leuenberger et al. [41], and has also been seen in EuO [86]. For the two samples with higher resistivity the resistance reaches a minimum as the temperature is decreased to $25 \mathrm{~K}$ before beginning to increase rapidly again. Thus it is concluded that GdN is an intrinsic semiconductor in the ground state, in disagreement with the observation by Leuenberger et al. in [41]. In that paper they offer two possible causes for their observation of a metallic ground state: an intrinsic semiconductor-metal transition, or a transition due to the spin-split conduction band crossing the high concentration of donor impurity levels that may be present. The second explanation is more likely.

It is important to note that extrinsic effects can cause a metallic sample to appear semiconducting. In particular insulating grain boundaries can 
make an intrinsic metal appear semiconducting. If this were the case then the measured resistivity should vary as a function of the average crystallite size. In fact the resistivity of the TED samples grown with different $\mathrm{N}_{2}$ base pressures varies by over four orders of magnitude, while the crystallites do not noticeably differ in size. Further evidence for the intrinsic semiconducting nature of GdN is gained by the lack of a Drude peak in optical transmission measurements, presented in Section 6.5.

\subsection{Magnetism}

Magnetization data were obtained from films grown by both TED and PLD. Figures 6.2 (a) and (b) show temperature- and field-dependent magnetization, respectively. In both cases the external magnetic field was aligned in-plane. The temperature dependent magnetization shows both ZFC and FC curves and the applied field was $0.05 \mathrm{~T}$ and $0.01 \mathrm{~T}$ for the TED and PLD films, respectively. The field dependent hysteresis loops were taken at a temperature of $5 \mathrm{~K}$.

The magnetization, in Bohr magnetons per gadolinium ion $\left(\mu_{B} / \mathrm{Gd}\right)$, was calculated from the measured magnetization in electromagnetic units (emu). For the TED sample, where the volume of the film was known from RBS, a direct conversion was done. The resulting saturation moment, $6.4 \pm 0.6 \mu_{B} / \mathrm{Gd}$ at $6 \mathrm{~T}$, is in agreement with the moment of $7.0 \mu_{B} / \mathrm{Gd}$ expected for the lowest energy $\mathrm{Gd}^{3+}$ multiplet. For the PLD film, which saturates very easily, but for which the exact film thickness was unknown, the magnetization was normalized on the assumption that the saturation moment is $7.0 \mu_{B} / \mathrm{Gd}^{1}$

Turning to the temperature dependent data first, both films show the qualitative features of a ferromagnet and in both cases a Curie-Weiss fit to the magnetic response above $100 \mathrm{~K}$ gives a transition temperature of close

\footnotetext{
${ }^{1}$ This was proved reasonable by extracting the volume of the sample from the calibrated moment. For a sample area of $10^{-5} \mathrm{~m}^{2}(2 \mathrm{~mm} \times 5 \mathrm{~mm})$ the extracted film thickness is $200 \mathrm{~nm}$, consistent with the measured thickness of similar films.
} 

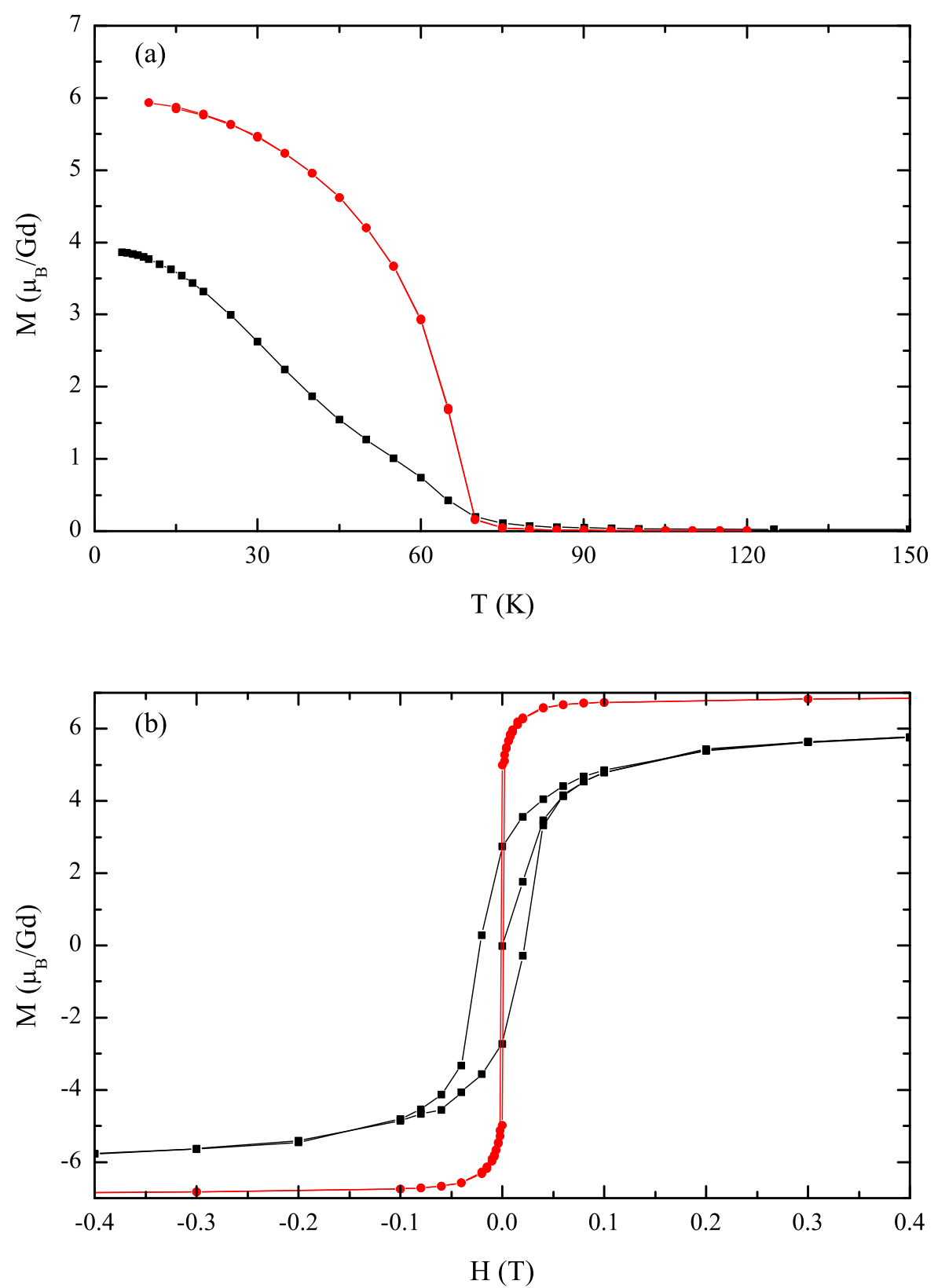

Figure 6.2: Magnetization of GdN as a function of (a) temperature, and (b) applied field. Films grown by TED (black squares) and PLD (red circles) are shown. The temperature dependent data were obtained with the TED and PLD films in applied fields of $0.05 \mathrm{~T}$ and $0.01 \mathrm{~T}$, respectively. 
to $70 \mathrm{~K}$. For both samples the magnetization, taken in an applied field on the order of tens of $\mathrm{mT}$, increases rapidly below $70 \mathrm{~K}$. Comparing the films, the upturn of the PLD sample is significantly sharper, indicating a more homogeneous film.

The maximum moments at low temperature are roughly 4 and $6 \mu_{B} / \mathrm{Gd}$ for the TED and PLD films, respectively, significantly less than the saturation moment of $7.0 \mu_{B} / \mathrm{Gd}$. This is explained by considering the field dependent data, in Fig. 6.2 (b), which was taken at $5 \mathrm{~K}$. Both films show hysteresis and saturation. From the hysteresis, coercive fields of $0.022 \mathrm{~T}$ and $0.001 \mathrm{~T}$ and remanences of $2.74 \mu_{B} / \mathrm{Gd}$ and $5.02 \mu_{B} / \mathrm{Gd}$ are extracted for the TED and PLD films, respectively. At an applied field of $0.05 \mathrm{~T}$ the TED film has a moment of roughly $4 \mu_{B} / \mathrm{Gd}$ and at $0.01 \mathrm{~T}$ the PLD film has a moment of $6 \mu_{B} / \mathrm{Gd}$. This is in excellent agreement with the moments seen in the temperature dependent data.

The relatively high $\mathrm{T}_{C}$ and small coercive fields of these films are as expected for O-free GdN from previous experiments [78, 39], although recent theoretical results have raised some question about the nature of ferromagnetism in GdN [91, 92]. In the case of the PLD film the coercive field of just $0.001 \mathrm{~T}$ is lower than any previously reported. A strong correlation between increasing crystallite size and decreasing coercive field is detailed in Ref. [82]. In the rare-earths, where coupling to the crystal field is often weak [13, 93], and in GdN in particular, where the half-filled $4 f$ shell has no orbital moment, it is likely that the coercivity is largely related to shape anisotropy introduced by small crystallites.

\subsection{Transport and Magnetism}

We now turn to a combined analysis of a GdN film grown by TED. Figure 6.3 shows the resistivity and magnetization of the film measured as a function of temperature. The qualitative features are the same as described above. Of interest is the fact that the resistivity peaks at $68 \mathrm{~K}$, coinciding with 


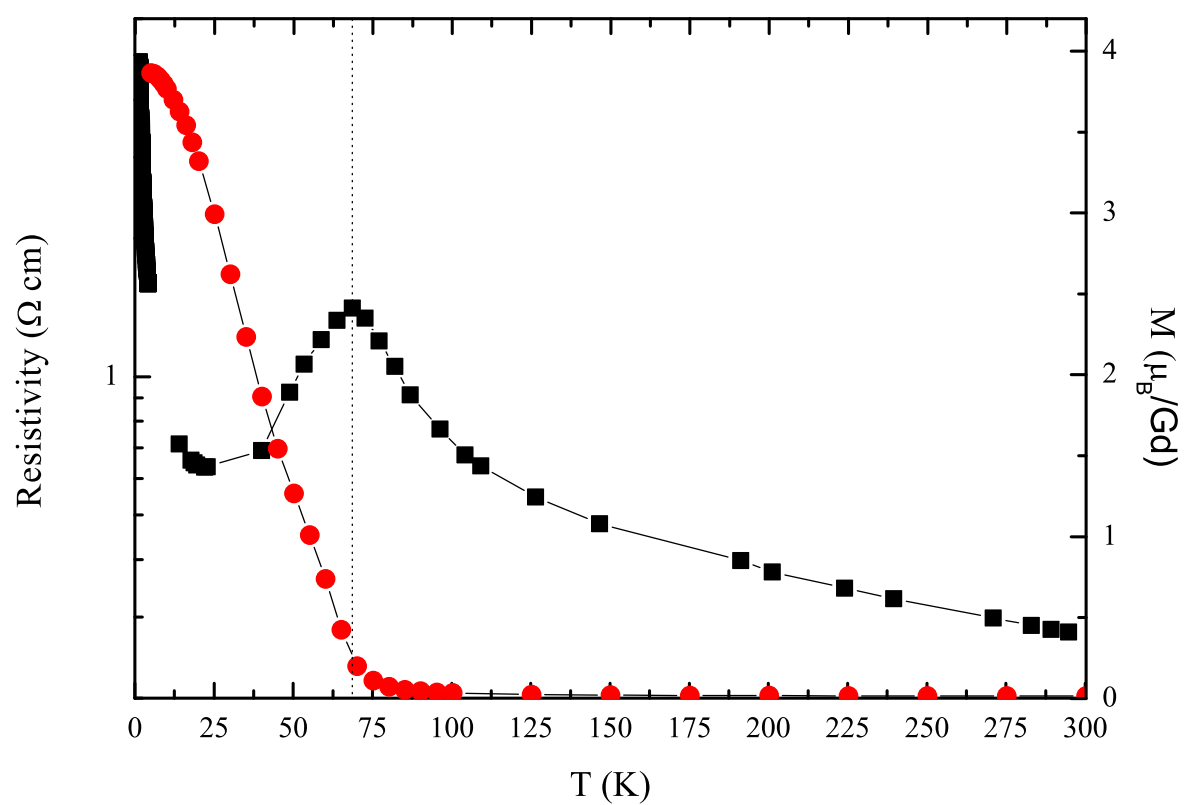

Figure 6.3: Resistivity (black squares) and magnetization (red circles) measured as a function of temperature.

the ferromagnetic transition temperature. This strongly suggests that the change in resistivity is effected by the ferromagnetic transition - there is a link between electronic and magnetic properties, as desired for spintronic applications. The size of the drop in resistivity (roughly $50 \%$ ) is less than seen in other magnetoresistive systems, like $\mathrm{EuO}$ [81], but too large to be due to a reduction in magnetic scattering alone. From the low temperature resistivity data it is apparent that GdN is semiconducting in the ferromagnetic ground state, but with a smaller gap than at ambient temperature.

A qualitative explanation for the experimental data can be obtained by considering the splitting of the majority- and minority-spin states in the ferromagnetic ground state. Calculations show that the direction of the splitting is opposite for occupied and unoccupied states ([54], see Fig. 6.5 below). Since the Gd $4 f$ moments are randomly oriented in the paramagnetic state the majority and minority bands are effectively "averaged" (see Section 6.5), implying that the majority gap narrows in a transition to the ferromagnetic 


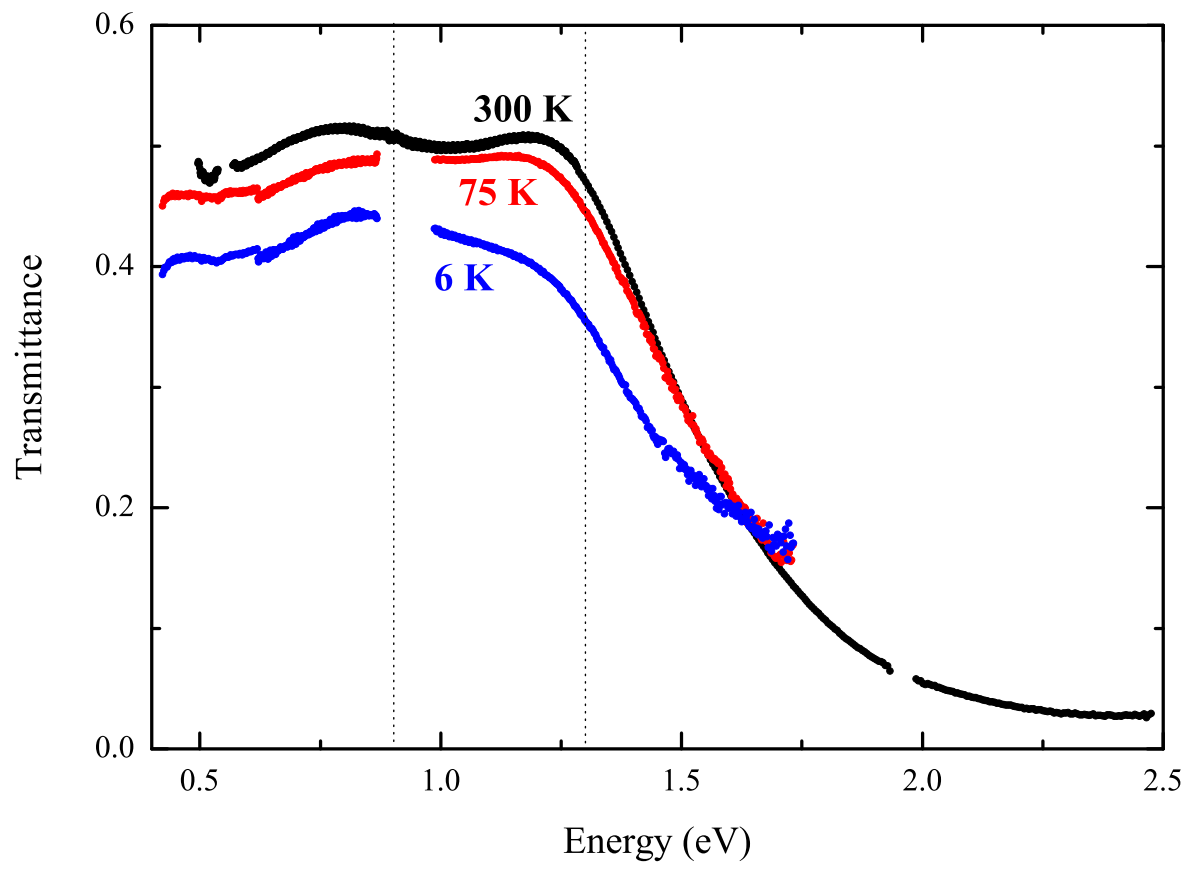

Figure 6.4: Optical transmission as a function of incident light energy for a GdN sample at different temperatures. A clear absorption edge is seen near $1 \mathrm{eV}$ in each case.

state [94], and that transitions at the absorption edge are between majority spin bands. As mentioned above, sample conductivity is most likely mediated by defect states in the gap. If the separation between these states and the CBM scales with the intrinsic gap then the experimental results are as expected.

\subsection{Optical Transmission}

Further evidence for the above interpretation - that GdN is semiconducting in both the paramagnetic and ferromagnetic states, and that majorityminority band splitting in the ferromagnetic state leads to a decrease in the effective gap size - along with a measurement of the absolute size of 
the gap was obtained from optical transmission measurements. The energydependent transmission data was obtained by FTIR spectroscopy of a film grown by TED and capped with GaN. Figure 6.4 shows the transmittance at 6, 75, and $300 \mathrm{~K}$. All scans show clear absorption edges near $1 \mathrm{eV}$. Below the edge, transmittance is flat except for interference fringes caused by the GaN and GdN layers (substrate interference fringes with a periodicity of about $6 \mathrm{~cm}^{-1}$ have been smoothed out of the data). There is no evidence in these samples for a Drude response that would indicate a high concentration of free carriers.

By extrapolating the transmission below the edge, and from the inflection point on the edge, the $300 \mathrm{~K}$ data was estimated to have an optical gap of $1.31 \pm 0.03 \mathrm{eV}$. At $75 \mathrm{~K}$ (immediately above $\mathrm{T}_{C}$ ) there is little change in the transmittance relative to the $300 \mathrm{~K}$ data, showing only small changes in the interference pattern attributed to thermal contraction and the temperature dependence of the optical constants in the film and substrate. Most importantly the interband edge is at the same energy. In contrast at $6 \mathrm{~K}$, in the ferromagnetic ground state, there are significant changes in the magnitude of the subgap transmission, and in the position of the edge. The original absorption edge is somewhat attenuated and a very clear shoulder extends to a new edge at lower energy. Using the same extrapolation technique the edge is found to be at $0.90 \pm 0.03 \mathrm{eV}$. Reduced transmission below the edge is attributed to increased reflectivity caused by a higher refractive index due to the narrower gap.

By assuming that transmission is proportional to $T_{0} e^{-\eta d}$, where $T_{0}$ is the transmission in the subgap region, and $d$ is the film thickness, it is possible to estimate $\eta$, the absorption coefficient. Above the gap the absorption coefficient rises steeply to over $10^{4} \mathrm{~cm}^{-1}$. Such strong absorption is typical of interband direct transitions, orders of magnitude larger than seen in indirect transitions.

An early study by Busch et al. [17], based on diffuse reflection measurements of powder samples, saw some evidence for an optical gap at $0.98 \mathrm{eV}$. It 
is interesting that the same paper reported what was regarded as anomalous optical data on one sample of $\mathrm{GdN}$, apparently with a lower-than-typical N deficiency and thus weaker free carrier absorption, in which the diffuse absorption showed an onset close to that reported here. The paper also made the claim that there was no shift of the edge in the ferromagnetic phase, but that the subgap absorption increased significantly. Although no low temperature data were presented, that description suggests a behaviour similar to the present results, except that the data were apparently not extended to the red-shifted edge.

More recently there has been a report of band shifts in GdN observed in XAS taken at the Gd L-edge [40]. The DOS at the Gd $\mathrm{L}_{2}$ edge was seen to red-shift by a few hundred meV in the ferromagnetic state. Those data measure the peak in the CB DOS relative to the Gd $2 p_{1 / 2}$ core level, so they are not directly comparable to the present interband data. However, it is interesting that the red-shift is of very similar magnitude to the interband edge red-shift reported here.

The data presented here provides strong evidence that GdN is an intrinsic semiconductor, and has optical (direct) band-gaps of $1.3 \mathrm{eV}$ and $0.9 \mathrm{eV}$ in the room temperature paramagnetic and ferromagnetic ground states, respectively.

\subsection{Band Structure}

By now we have a picture of GdN as a semiconductor, with a ferromagnetic semiconducting ground state. Additionally, the low temperature decrease of both the activation energy in the transport data and of the absorption edge in the optical transmittance data suggest that the band-gap is narrower in the ferromagnetic ground state. These results can be used to guide theoretical calculations.

In a calculation that follows directly from the work of Larson et al. [13] we have used DFT with the LSDA $+U$ approximation to determine the band 


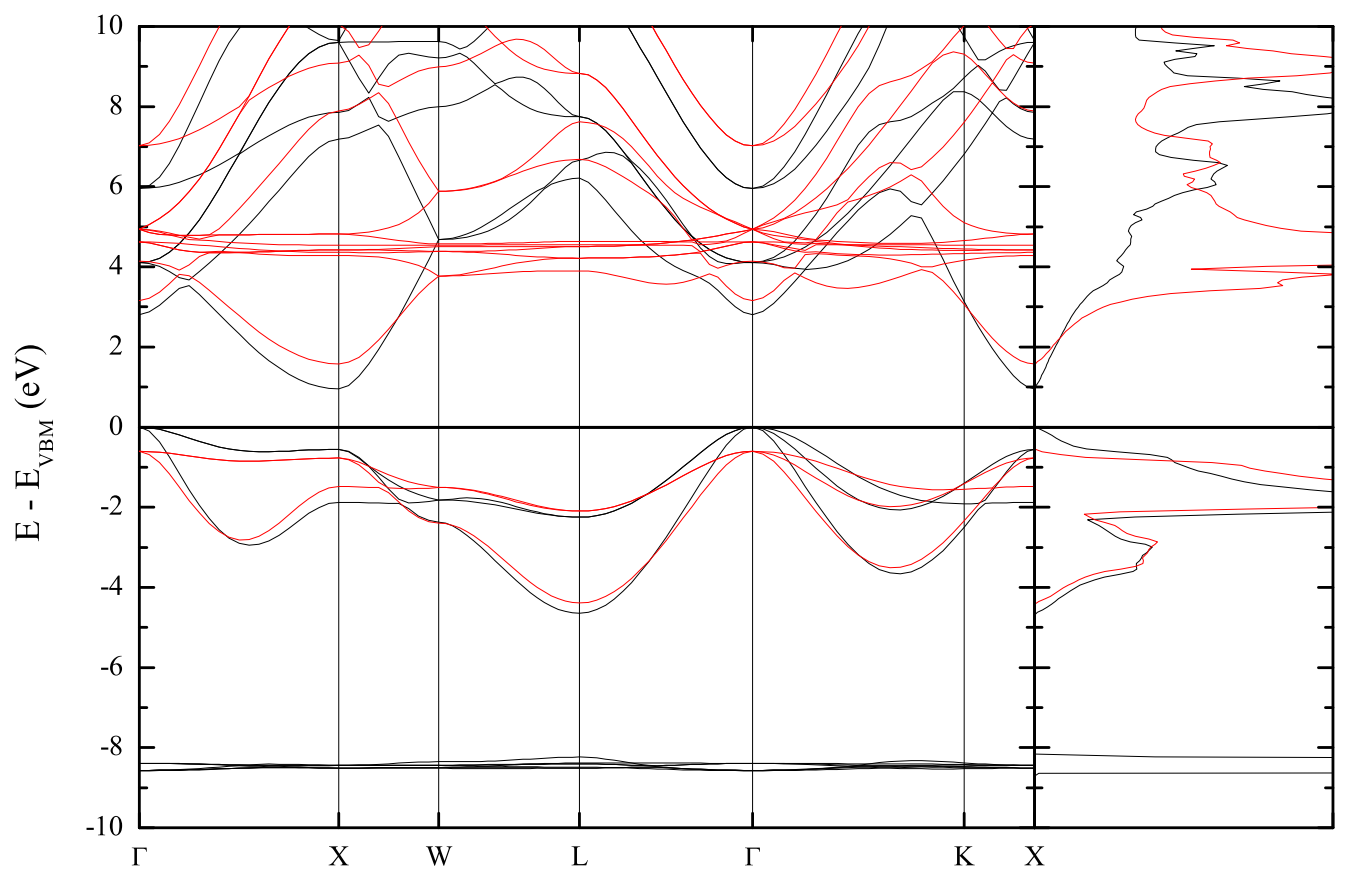

Figure 6.5: $\mathrm{LSDA}+U$ band structure and DOS calculated with $U_{d}=8.0 \mathrm{eV}$ and $U_{f}=9.2 \mathrm{eV}$. Majority- (black lines) and minority-spin (red lines) bands are isolated.

structure of GdN. An overview of the DFT is provided in Section 3.2, and details of the computational framework can be found in Section 3.4. The best available data for the position of the $4 f$ orbitals were considered in Ref. [13], therefore the same Hubbard- $U_{f}$ parameters are used in the present calculations $\left(U_{f}=9.20 \mathrm{eV}\right.$ and $\left.J_{f}=1.20 \mathrm{eV}\right)$. With $U_{d}$ set to zero (no gap correction) GdN is found to be a half-semimetal (a semimetal in the majority states only) and is in principle a conductor with perfect spin-polarization [89]. On the other hand a positive $U_{d}$ value results in a semiconducting band structure and, with careful tuning, excellent agreement with the optical transmission data is obtained [94].

Figure 6.5 shows the GdN spin-resolved band structure plotted along directions of high symmetry in the Brillouin zone, and the corresponding 
DOS. The zero of energy is set to be at the VBM, above which there is a clear gap before the CBM. The VBM is at $\Gamma$ and the CBM at $\mathrm{X}$, therefore $\mathrm{GdN}$ is an indirect semiconductor. The smallest direct gap, which should correspond to the optical absorption edge, is found at X. As expected for a ferromagnetic material, the majority- and minority-spin bands are spin-split due to the exchange interaction with the spin-polarized $4 f$ levels. $^{2}$ Relative to the minority bands, the occupied majority bands are at a higher energy and the unoccupied majority bands are at a lower energy. This means that both the indirect and direct minority gaps are significantly larger than the majority gaps.

To compare with optical transmission data we measure the size of the direct gap at X. For a first approximation between theory and experiment, we assume the gap in the ferromagnetic state is given by the majority gap, and in the paramagnetic state by the average of the majority and minority gaps. This is justifiable since in the high temperature state spins should not be ordered. In a remarkable agreement between theory and experiment we find that a choice of $U_{d}=8.0 \mathrm{eV}$ leads to a majority-spin gap of $0.91 \mathrm{eV}$ and an average gap of $1.30 \mathrm{eV}$.

It is important to consider whether the representation of bands in the paramagnetic state as the average of the spin-resolved bands is realistic. Ref. [94] details two calculations designed to test this. The calculations were performed on a 32 atom supercell using a noncollinear code that allows the moments of each of the $4 f$ orbitals to be aligned in different directions. The spins of the $16 \mathrm{Gd}$ atoms were first ferromagnetically aligned, and then noncollinear randomly aligned. The ferromagnetic calculation was necessary as a control since the framework for the noncollinear calculation is not as well tested as the standard LDSA $+U$ calculation. In the ferromagnetic noncollinear calculation the average of the spin up and spin down direct gaps was $1.35 \mathrm{eV}$. This is sufficiently similar to the LSDA $+U$ calculation (where

\footnotetext{
${ }^{2}$ Fig. 6.5 does not show the seven occupied $4 f$ bands, which are located at roughly $8 \mathrm{eV}$ below the VBM. The unoccupied $4 f$ bands, can be seen at roughly $5 \mathrm{eV}$.
} 
the average direct gap was $1.30 \mathrm{eV}$ ) that we can trust the noncollinear code. In the calculation where the $4 f$ moments are randomly aligned, the direct gap was $1.30 \mathrm{eV}$, only $0.05 \mathrm{eV}$ less than the ferromagnetic calculation. In fact the discrepancy can be attributed to the finite supercell size in the noncollinear calculation - the residual magnetic moment of the randomly oriented spins was $1.44 \mu_{B} / \mathrm{Gd}$. Therefore the heuristic of averaging the spins of the LSDA $+U$ calculation estimate the paramagnetic direct gap seems plausible. We conclude that $\mathrm{GdN}$ is an indirect semiconductor with an indirect gap of $0.43 \mathrm{eV}(0.98 \mathrm{eV})$ in the ferromagnetic (paramagnetic) state.

\subsection{X-ray Spectroscopy}

X-ray spectroscopy was performed on a GdN sample grown by PLD and capped with YSZ. Experimental details are described in Section 4.4. The PLD samples were chosen for the technical reasons detailed in Section 5.3 and are known to contain Gd droplets and more nitrogen defects than the TED samples, from which the optical band gap was extracted. The pertinent differences in their transport and magnetic properties are discussed in Sections 6.2 and 6.3. However, by definition, spectroscopy at the N K-edge measures the local electronic structure around nitrogen sites. Thus there is good reason to believe defect states, in the concentrations observed for the PLD sample, will have minimal impact on spectra.

\subsubsection{XAS and XES}

Figure 6.6 shows the GdN N K-edge XES and XAS. The XES is due to the $\mathrm{N} 2 p$ bands that form the upper VB. A shoulder on the low energy side of the peak is more clearly resolved than in the SmN and DyN XES we have previously reported [95], which we attribute to the superior crystalline quality of the GdN sample used in the present study. The XAS signal measures the $\mathrm{N} p$-projected PDOS of the $\mathrm{CB}$, which can largely be interpreted as the $p$ 


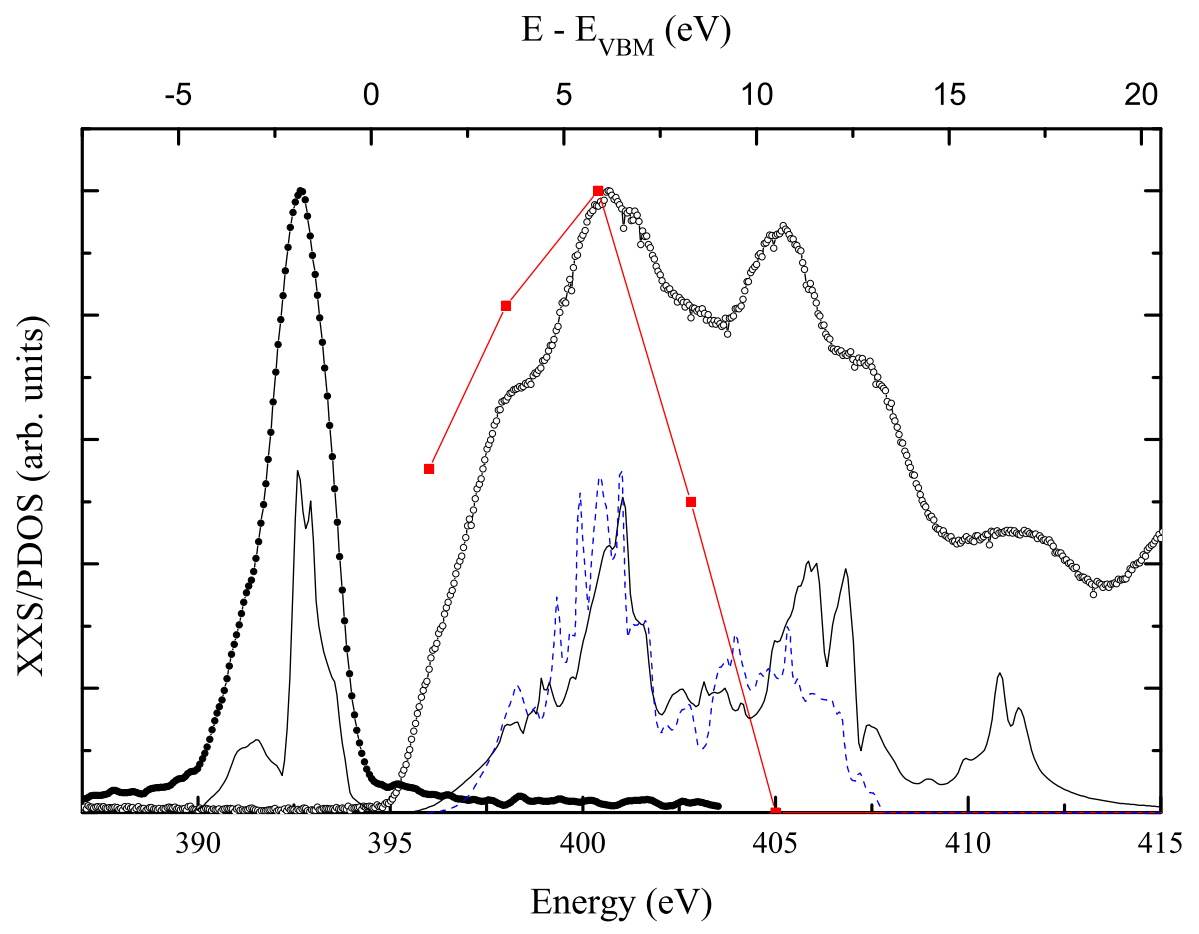

Figure 6.6: N K-edge XES (solid circles) and XAS (empty circles). Also shown is the calculated PDOS. Both the standard PDOS (black line), and a core-hole calculation (dashed blue line) are shown. The coherent fraction of each RXES scan is shown at the excitation energy used to generate it (red squares).

component of the tails of the $5 d$ and $6 s$ orbitals overlapping onto the $\mathrm{N}$ sites.

In GdN the occupied Gd $4 f$ bands are calculated to sit roughly $8 \mathrm{eV}$ below the VBM. The occupied $\mathrm{Zn} 3 d$ bands in $\mathrm{ZnO}$ are in a similar position; we recently reported evidence for the observation of $\mathrm{O} 2 p-\mathrm{Zn} 3 d$ hybridization in XES [96] but see no evidence for a similar effect here. The Gd $4 f$ levels seem to behave very much as core levels, as is confirmed by the Gd M-edge XAS in Section 6.7.3.

The experimental spectra are compared with PDOS extracted from the $\mathrm{LSDA}+U$ calculation detailed above. For comparison to the room tempera- 
ture experimental data (well above the ferromagnetic transition temperature) the PDOS is an average of the contributions from both spin orientations. The PDOS has been rigidly shifted so that the main VB peak matches with experiment. This yields excellent agreement with the XES, accurately locating the main peak and the lower energy shoulder.

Analysis of the XAS is complicated by the presence of a core-hole in the final state. We include in Fig. 6.6 the PDOS of the unoccupied states obtained from a core-hole calculation. The single improvement compared with experiment seems to be the appearance of a small peak at $398.2 \mathrm{eV}$, consistent with the shoulder seen in the XAS. However, compared with the ground state PDOS there are no big shifts. The main change seems to be in the peaks at around $406 \mathrm{eV}$; in the core-hole calculation these are strongly attenuated. This is also the main effect observed in core-hole calculations for SmN and DyN in Section 7.5 and Ref. [95].

It is interesting to note that no core-hole shift to the ground state PDOS is necessary to obtain energetic agreement with the main XAS peaks, and that the $405 \mathrm{eV}$ peak does not appear to be strongly attenuated. Since the core-hole shift has been shown to be important in similar experiments on many other materials [97], it is important to consider why it is not observed here. We note the high n-type conductivity of the sample $\left(\sim 10^{20} \mathrm{~cm}^{-3}[82]\right)$. It may be that the carriers screen the core-hole potential from the largely Gd $5 d$ and $6 s$ conduction band states.

\subsubsection{RXES}

As described in Section 4.4.3, the coherent fraction of RXES can be used to interrogate specific regions of the band structure. Figure 6.7 (a) shows RXES obtained at a number of excitation energies. The excitation energy of each spectrum is labelled and is also indicated by the bars on Figs. 6.6 and 6.8. For RXES obtained with excitation energies below $405.0 \mathrm{eV}$ there is a clear evolution in the spectra, including shifts in the main peak energy and changes in the intensity of the tail near $391.0 \mathrm{eV}$. On the other hand the 

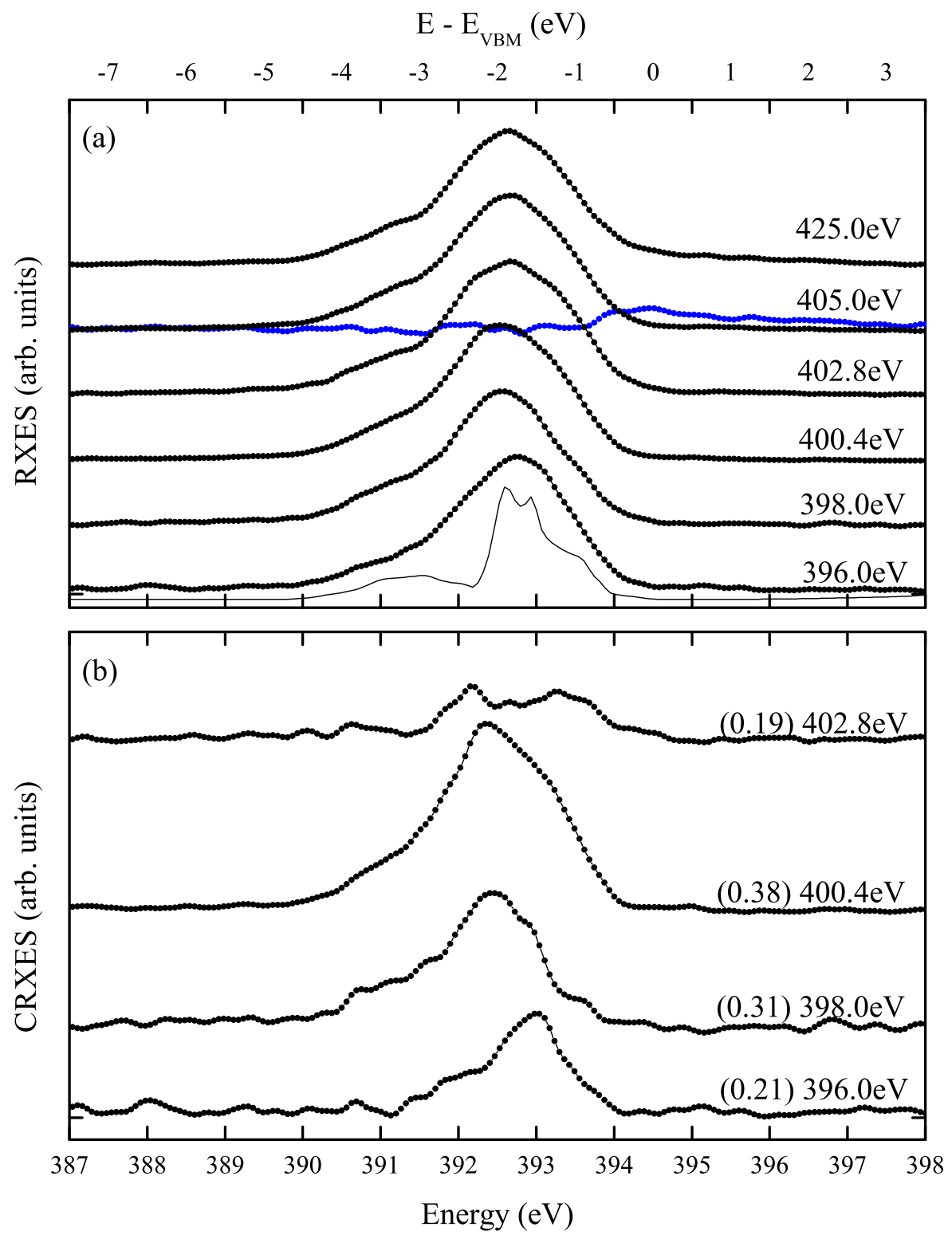

Figure 6.7: (a) RXES spectra (solid circles) taken at a number of incident energies (labelled). The result of subtracting the $425.0 \mathrm{eV}$ RXES from the $405.0 \mathrm{eV}$ RXES (blue circles) and the N p-projected PDOS (solid line) are also shown. (b) The coherent fraction RXES (solid circles). Labels indicate the excitation energy, and also the coherent contribution. 
highest energy spectrum $(425.0 \mathrm{eV})$ is far enough from the absorption edge that it is considered (incoherent) XES.

Typically the coherent part of RXES (CRXES) is obtained by subtracting as much of the XES from a spectrum as possible while ensuring that no part of the resulting spectrum drops below zero [72]. However, in the $425.0 \mathrm{eV}$ spectrum an anomalous contribution to the signal appears on the high energy side of the XES, meaning that the conventional approach will not work. Instead the $405.0 \mathrm{eV}$ RXES spectrum is used as the XES. This is proved reasonable by subtracting the $405.0 \mathrm{eV}$ RXES from the $425.0 \mathrm{eV}$ XES. The difference is shown along with the RXES in Fig. 6.7 (a). It is clear that the $405.0 \mathrm{eV}$ and $425.0 \mathrm{eV}$ XES are similar, except for the high energy anomaly.

It is interesting to consider where the band-tailing on the $425.0 \mathrm{eV} \mathrm{XES}$ could come from. One possibility is that a high density of defect states in the gap begins to contribute intensity to the signal. However, it is difficult to understand why this signal would be so heavily dependent on excitation energy. Another possibility is that the signal comes from an external source. The GdN film was passivated with a YSZ capping layer, through which the measurements were made. Emission due to relaxation from valence states into the zirconium $\mathrm{M}_{1}$ core-hole, which has a binding energy of $430.3 \mathrm{eV}$ [66], could be the source.

Figure 6.7 (b) shows the CRXES obtained as a result of the XES subtraction. The 396.0, 398.0, 400.4, and $402.8 \mathrm{eV}$ CRXES (now referred to as $\mathrm{C}_{396.0}, \mathrm{C}_{398.0}, \mathrm{C}_{400.4}$, and $\mathrm{C}_{402.8}$, respectively) all show substantial differences from the XES. Briefly, $\mathrm{C}_{396.0}$ shows added weight to the peak at $393 \mathrm{eV}$ while the $\mathrm{C}_{398.0}$ has an enhanced signal at $392.4 \mathrm{eV}$, and also some extra intensity near $391 \mathrm{eV} . \mathrm{C}_{400.4}$ has the strongest coherent component and contains weight from all three peaks. Meanwhile, the $402.8 \mathrm{eV}$ RXES is very similar to the $\mathrm{XES}$, as evidenced by $\mathrm{C}_{402.8}$, which is very weak.

As described in Section 4.4.3, simple descriptions of RXES assume that electron-phonon and electron-electron scattering will increase with excitation energy and weaken the coherent contribution to the scattering [72]. The 
strength of the GdN coherent contribution is calculated by integrating over each CRXES spectrum and dividing by the integrated XES; the labels in Fig. 6.7 (b) indicate the extracted value. The contribution does not decrease monotonically. In fact it was shown by Strocov et al. [97] that the coherent contribution in GaN scales with XAS intensity. The normalized GdN coherent contribution is plotted in Fig. 6.6, where similar scaling with the XAS is observed.

The variation of the CRXES can be fully described by the KramersHeisenberg formula within an energy band model [72, 98], as seen for the Be chalcogenides by Eich et al. [99] and CdS by Weinhardt et al. [100]. However, to a first approximation the shifts in the CRXES can be understood directly from the calculated band dispersion using the conservation of electron momentum, as shown for GdN by Strocov et al. [97] and ZnO by Preston et al. $[96]$.

Figure 6.8 shows the calculated band structure of GdN on the left, and the $\mathrm{N} p$-projected PDOS overlaid with the XAS on the right. The horizontal bars on the XAS indicate the CRXES excitation energies. The vertical bars on the band structure indicate the relative strength of the $\mathrm{N} p$ character along each band. For example the large bars on the valence bands highlight their strong $\mathrm{N} p$-like character. On the other hand the calculation shows that between $\Gamma$ and $\mathrm{X}$ the highly dispersive bands that form the CBM have almost no p-like character. The CRXES is $\mathbf{k}$-conserving, therefore it is expected that emission will be restricted to areas of the Brillouin zone that have strong $p$-like character at the energy of the incident light. This means that any CRXES from x-rays incident with an energy of $398.5 \mathrm{eV}$ or less will access states only between $\mathrm{X}$ and $\mathrm{W}$.

$\mathrm{C}_{396.0}$ has an excitation energy of $396.0 \mathrm{eV}$, and thus represents emission from states near $\mathrm{X}$. The valence bands in this region are constrained to a narrow region close to the VBM, fully consistent with the increase in intensity on the high energy side of $\mathrm{C}_{396.0}$. Likewise, the observed $\mathrm{C}_{398.0}$ shifts are understood to be due to emission from close to $\mathrm{W}$ where, relative to $\mathrm{C}_{396.0}$, the 


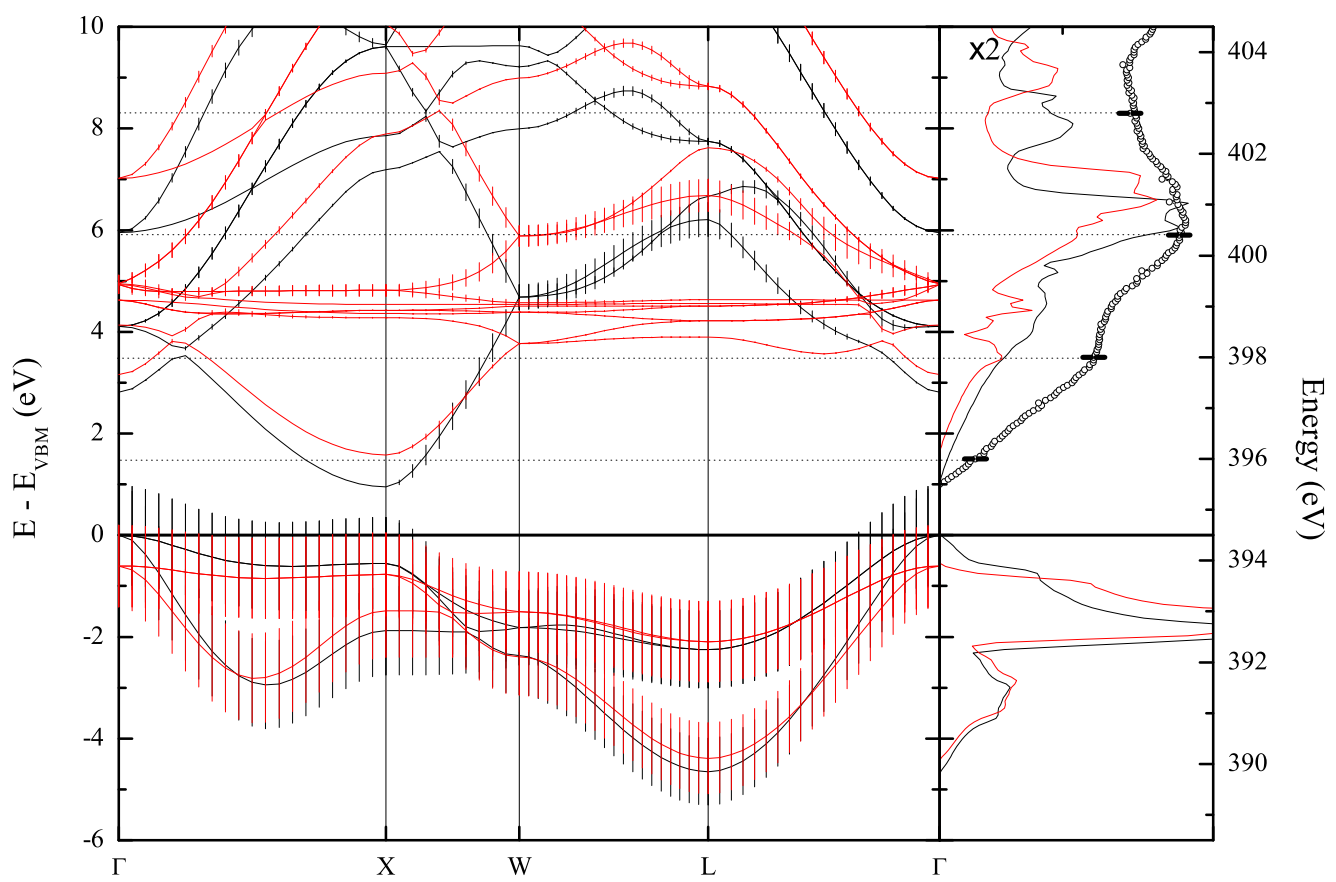

Figure 6.8: Calculated GdN band structure, resolved into majority- (black) and minority-spins (red); vertical bars indicate $p$-like character of the band. Right: Calculated PDOS (lines) and XAS (open circles); horizontal bars indicate RXES excitation energies.

valence bands have dispersed to lower energy. At $400.4 \mathrm{eV}$ there is strong $p$ like character along W-L- $\Gamma$. Near $\mathrm{L}$ the valence band has contributions down to $390.5 \mathrm{eV}$ and this is reflected in the intensity on the low energy side of the $\mathrm{C}_{400.4}$ spectrum. The intensity observed on the high energy side is attributed to the $p$ character of the bands near $\Gamma$. The agreement between experiment and theory at this excitation energy allow us to confidently assign the area around L as the part of the Brillouin zone from which the main XAS peak originates. At $402.8 \mathrm{eV}$ relatively little $p$-like character is observed; weak contributions seem to be evenly distributed across the band structure. This is consistent with the very small coherent fraction extracted from $\mathrm{C}_{402.8}$. 


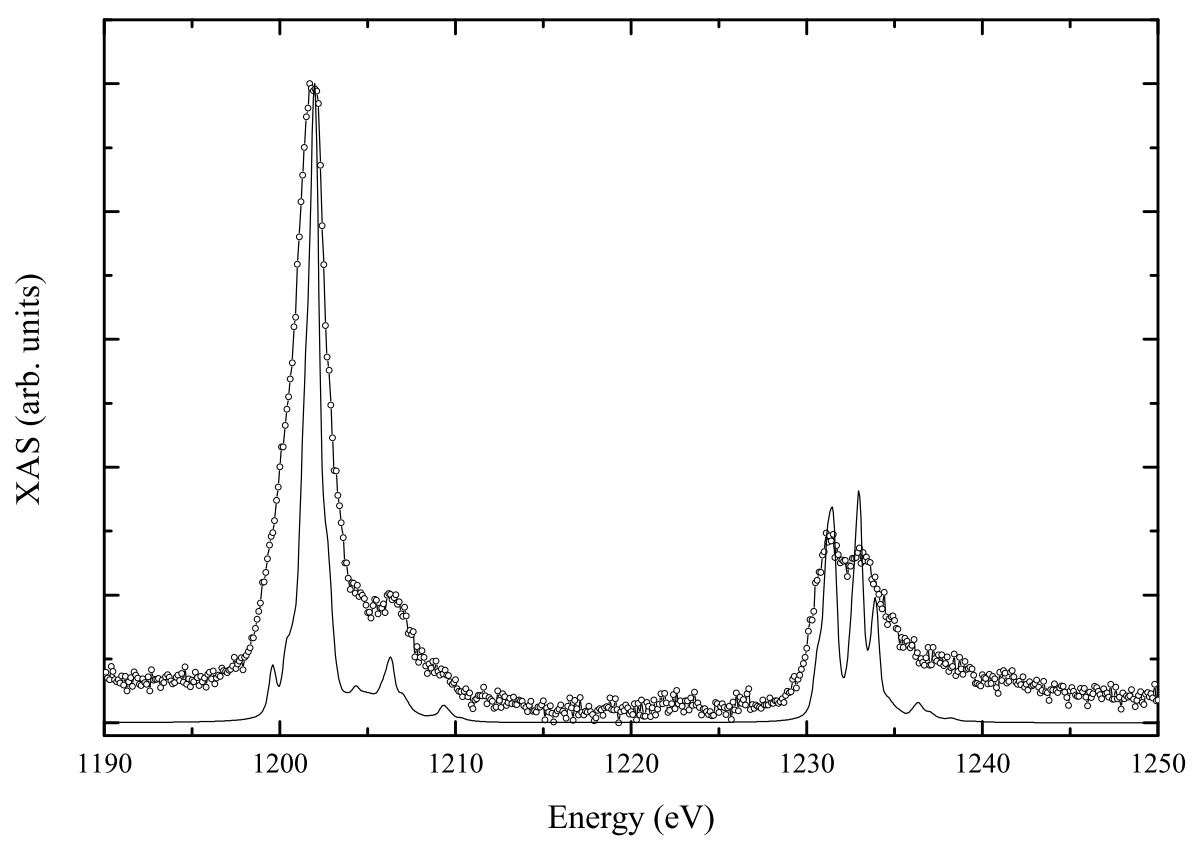

Figure 6.9: Gd M-edge XAS (symbols) compared with theory (solid line). The similarity between theory and experiment highlights the atomic-like nature of the $4 f$ orbitals.

\subsubsection{Gd M-edge}

XAS at the Gd M-edge was also obtained. Figure 6.9 shows the spectrum. The result of an atomic multiplet calculation, detailed in Section 3.1, is also shown in the figure. Excellent agreement is seen with experiment indicating that the majority of the $\mathrm{Gd} 4 f$ wavefunctions are on the $\mathrm{Gd}$ sites. To account for screening in atomic calculations it is common to scale the electrostatic and exchange parameters to roughly $80 \%$ of their value as calculated by the HFA (see Section 3.3.1) in order to reach best agreement with experiment. The precise values used in the calculations in this thesis are tabulated in Ref. [47].

It is noted in Section 3.4.1 that the $U_{f}$ and $J_{f}$ parameters used in the LSDA $+U$ calculation are strongly related to the Coulomb integrals obtained from HFA calculations. In Ref [13] Larson et al. used published XPS and 
Bremsstrahlung isochromat spectroscopy (BIS) of the Gd pnictides to experimentally determine $U_{f}=0.3 F^{0}$. On the other hand $J_{f}$ was calculated using Equation 3.26 and unscreened values for the Slater-Coulomb integrals. Given the superior agreement of the $80 \%$ screened values with the Gd M-edge XAS it makes sense to alter $J_{f}$ to a screened value. In that case $J_{f}=0.96 \mathrm{eV}(c f$., $J_{f}=1.20 \mathrm{eV}$ in Ref. [13]).

Recalculating the GdN band structure with this new value does not significantly alter the result. This is because $J_{f}$ determines the splitting between various occupied states and in the half-filled shell of Gd these states are degenerate. Still, screening of the HFA values should be considered in future numerical band structure studies based on the LSDA $+U$ framework.

\subsection{Conclusion and Outlook}

Together, the transport, magnetization, optical transmission, and x-ray spectroscopy data paint a cohesive picture of GdN as a semiconductor with a ferromagnetic ground state. Evidence for spin-splitting of the bands in the ferromagnetic state has been observed in the transport and optical transmission measurements. A tuned LSDA $+U$ calculation accurately predicts each of the experimental results.

The N K-edge XXS is also in agreement with the calculation. RXES measurements provide experimental evidence for the calculated dispersion at the CBM and locate the main XAS peak at L. This confirms that the calculation correctly locates the Gd $5 d$ and N $2 p$ bands and accurately predicts the crystal field splitting of the $5 d$ bands.

A remaining question is the location of the Gd $4 f$ levels, occupied and unoccupied. The atomic-like nature of the orbitals, which persists in the nitride, implies that they do not overlap significantly with $\mathrm{N} 2 p$ orbitals. This likely is why the $2 p-4 f$ hybrid is not observed in the $\mathrm{N}$ K-edge. Figure 6.10 shows the orbitally resolved PDOS of GdN, including the Gd $f$ and $d$-, and $\mathrm{N} p$-projected PDOS. Although the hybridization between the 


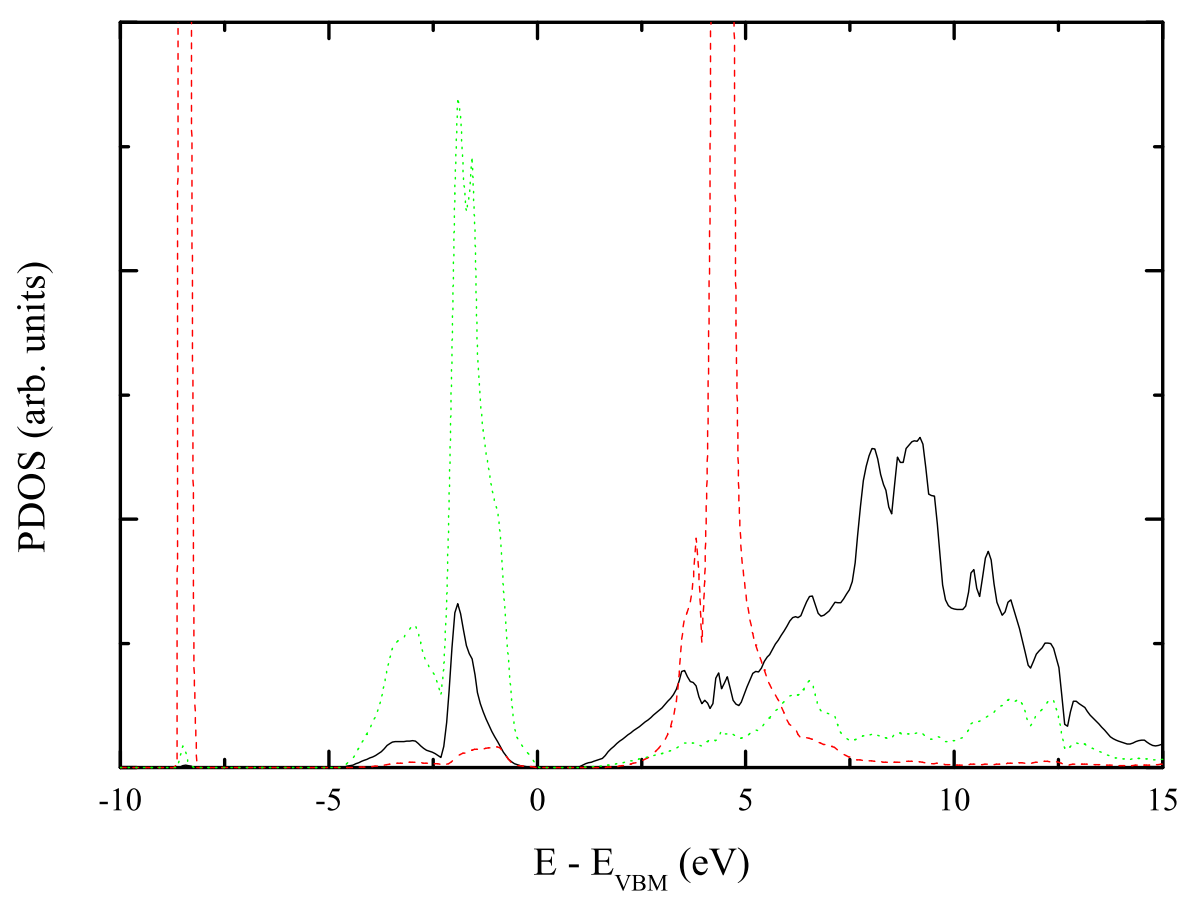

Figure 6.10: Calculated GdN PDOS. The Gd $d$ - (black) and Gd $f$ - (red), and N p-projected (green) PDOS are shown.

Gd $5 d$ and $4 f$ orbitals is not huge, it is much stronger than between $\mathrm{N} 2 p$ and Gd $4 f$. Although multiplet effects complicate analysis of the $p-d$ dipole transition it may be possible to obtain information on the energetic location of the unoccupied $\mathrm{Gd} 4 f$ levels from experiments at the $\mathrm{Gd} \mathrm{L}_{2,3^{-}}, \mathrm{M}_{2,3^{-}}$, or $\mathrm{N}_{2,3}$-edges. While some XAS at the $\mathrm{L}_{2,3}$-edge has been reported [40], the large energy of the transition $(\sim 7,200 \mathrm{eV})$ means the resolution is not good. The $\mathrm{N}_{2,3}$-edges are located at $\sim 280 \mathrm{eV}$ (see Table 4.1 ), an energy that is easily accessible by high resolution soft x-ray spectroscopy. Further experiments at this edge are desirable.

Beyond that, additional determinations of the electronic structure depend heavily on advances leading to the growth of high quality single crystals. This is essential for ARPES measurements that can be used to fully determine the $k$-resolved valence band. 



\section{Chapter 7}

\section{$\mathrm{SmN}$ and DyN}

\subsection{Motivation}

We turn now to the study of $\mathrm{SmN}$ and $\mathrm{DyN}$, where the rare-earth ion contains two fewer and two more $4 f$ electrons than $\mathrm{Gd}$, respectively. Relative to GdN the other ReNs have received far less attention, both theoretically and experimentally. Theoretical investigations have generally placed $\mathrm{SmN}$ as a half-metal and DyN as a semiconductor [36, 13]. In both of these calculations $\mathrm{SmN}$ is determined to have a near-zero magnetic moment due to cancellation between the spin and orbital components, although little is said of the energy differences between the antiferro- and ferro-magnetic ordered states.

There is little experimental work in the literature, and most of it is detailed in review articles by Hulliger [15] and Duan et al. [38]. Briefly, no data for thin films of either SmN or DyN exist, and the only data for bulk crystals are plagued by oxidization issues. Reports have generally pinned $\mathrm{SmN}$ as an antiferromagnet with a $\mathrm{T}_{C}$ below $20 \mathrm{~K}[30,29,16,15]$ although it is noted that measurements are complicated by the close spacing of the two lowest energy $\mathrm{Sm}^{3+}$ multiplets and the possibility that $\mathrm{Sm}$ could exist in the $2+$ state $[24,47]$. DyN, on the other hand, is quite clearly ferromagnetic [16, 15] although one report did label it as a ferrimagnet [29]. Temperature dependent resistivity measurements appear metallic, although this could easily be due 
to a high level of astoichiometry [21]. The energies of the optical band-gaps are not well placed, with DyN reported to have a direct gap of $0.91 \mathrm{eV}$ [15] or $2.6 \mathrm{eV}[21]$.

This chapter presents the result of electronic structure investigations of thin films of SmN and DyN which have been grown by TED. First, measurements of the transport and magnetic properties of both materials are reported. Next, the results of LSDA $+U$ band structure calculation are introduced. Finally, optical transmission data are used to determine the direct gap and XXS measurements are used to determine the $p$-projected PDOS. The experimental data are compared to the numerical results with some success. It is concluded that both $\mathrm{SmN}$ and DyN are ferromagnetic semiconductors, and an estimate is made of the optical gap of both materials. Further, it is proved that $\mathrm{SmN}$, uniquely for a semiconductor, has a near-zero moment in the ferromagnetic state.

\subsection{Transport and Magnetism}

Figures 7.1 (a) and (b) show the temperature dependent resistivity and magnetization of SmN and DyN, respectively. Turning first to the resistivity, the magnitude is a factor of 10 greater in DyN than in $\mathrm{SmN}$ and the temperature dependence more pronounced, although for both materials the behaviour is typical of a heavily doped semiconductor and any activation energy extracted from the data is orders of magnitude smaller than the calculated band gaps. Nevertheless the temperature dependence of the resistivity establishes that both materials are semiconducting over the entire temperature range (although with the same qualifications that were made for GdN in Section 6.2 - that grain boundaries could cause the same response).

The magnetic behaviour varies strongly between $\mathrm{SmN}$ and DyN. The magnetic response is very small in the case of $\mathrm{SmN}$, even though a field of $0.5 \mathrm{~T}$ was used in the FC measurement (compared to $0.05 \mathrm{~T}$ for DyN). This complicates analysis but has interesting implications. As a result it 
$\mathrm{T}(\mathrm{K})$
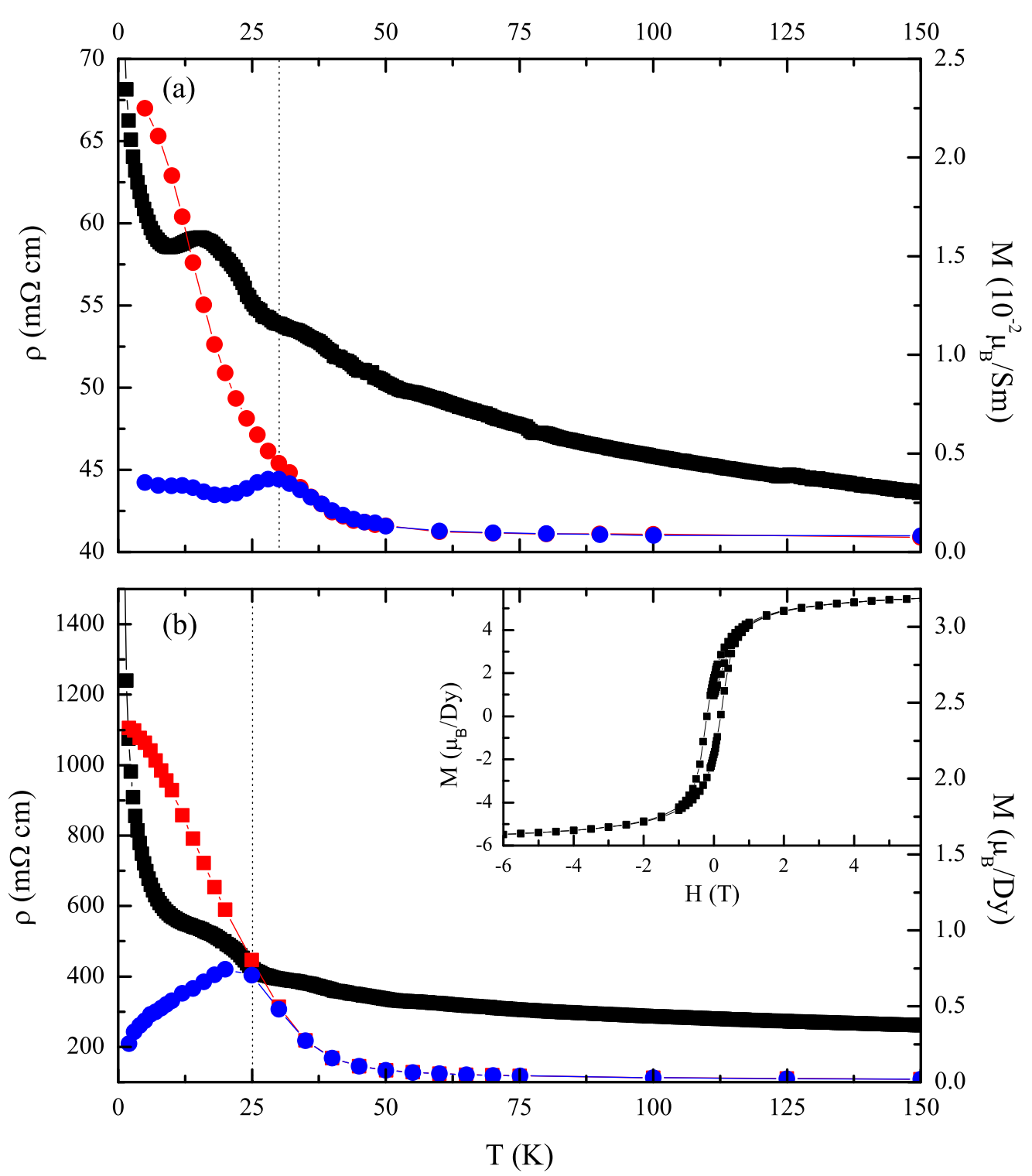

Figure 7.1: Resistivity (black squares) and magnetization (circles) as a function of temperature for (a) SmN and (b) DyN. The magnetization data were obtained after both ZFC (blue) and FC (red) preparations. Fields of $0.5 \mathrm{~T}$ and $0.05 \mathrm{~T}$ were used for $\mathrm{SmN}$ and $\mathrm{DyN}$, respectively. Inset: DyN hysteresis loop at $5 \mathrm{~K}$. 
was the subject of a detailed study by Meyer et al., the results of which will be detailed in the following section. Briefly, $\mathrm{SmN}$ has been found to be ferromagnetic with a $\mathrm{T}_{C}$ of close to $27 \mathrm{~K}$. DyN, on the other hand, is a relatively simple ferromagnet. The FC and ZFC magnetization curves show a divergence below $25 \mathrm{~K}$, indicating a ferromagnetic transition that is near the top of the range reported in the literature $[15,16]$. At $5 \mathrm{~K}$ the FC curve, measured in an applied field of $0.0500 \mathrm{~T}$, saturates to roughly $2.3 \mu_{B} / \mathrm{Dy}$ exactly as expected from the field sweeps taken at $5 \mathrm{~K}$ (see inset Fig 7.1 (b)). The sweeps show hysteresis with a coercive field of $0.2 \mathrm{~T}$ and a remanence of $2.3 \mu_{B}$ /Dy. At an applied field of $6 \mathrm{~T}$ the film saturates to a moment of nearly $6 \mu_{B} /$ Dy, significantly less than the $L+2 S z=10 \mu_{B} /$ Dy expected both from the Dy ${ }^{3+6} H_{15 / 2}$ ground state and the prediction of Larson et al. This is likely because the non-zero DyN orbital moment is quenched by a weak coupling to the cubic crystal field in the randomly oriented crystallites.

For both materials peaks in the resistivity are seen at temperatures close to the magnetic ordering temperature. ${ }^{1}$ This is strongly reminiscent of the behaviour observed for GdN, where exchange splitting of the bands is hypothesized to cause a reduction in the gap size in the ferromagnetic state leading to a reduction in the resistivity (see Section 6.4). There is no reason to believe the mechanism is different in $\mathrm{SmN}$ and DyN. However, in these samples the size of the drop in resistivity is too small to rule out other possible causes, one of which is a reduction in electron scattering due to increased magnetic ordering below $T_{C}$.

\subsubsection{SmN magnetism}

The magnetic response of $\mathrm{SmN}$ is very weak at all temperatures. The weak signal means that the typically insignificant substrate and capping layer contributions must be carefully accounted for. An understanding of the suscep-

\footnotetext{
${ }^{1}$ The peaks in the resistivity and magnetization data do not match exactly since they were obtained from samples that were known to have slightly different transition temperatures.
} 
tibility of $\mathrm{SmN}$ in the paramagnetic regime is also complicated by the fact that the $\mathrm{Sm}^{3+}$ ion ground state of ${ }^{6} H_{5 / 2}$ is only about $100 \mathrm{meV}(1500 \mathrm{~K})$ below the ${ }^{6} H_{7 / 2}$ first excited state. Indeed this is thought to be the cause of the disagreement between the experimental Sm M-edge XAS and the $\mathrm{Sm}^{3+}$ multiplet calculation reported by Thole et al. [47].

The small magnetic response is evident in the publication which first provided evidence suggesting SmN may be ferromagnetic [95]. The data include an approximately constant offset of about $0.004 \mu_{B} / \mathrm{Sm}$ associated with uncertainties in removing the background signal from the Si substrate, and no correction for the GaN capping layer. Nevertheless, the FC and ZFC curves separate at a low temperature which implies a magnetic transition at around $20 \mathrm{~K}$.

In recent work by Meyer et al. a more thorough analysis of thicker SmN films provides clear evidence for the near-zero moment ferromagnetic nature of $\mathrm{SmN}$ [101]. A number of different films of up to $400 \mathrm{~nm}$ in thickness were carefully analysed to reduce background signals. The FC and ZFC curves, shown in Fig. 7.1 (a), indicate a magnetization of less than $0.04 \mu_{B} / \mathrm{Sm}$ across the temperature range, despite the use of a field of $0.5 \mathrm{~T}$ in the FC measurement - at least an order of magnitude greater than needed to saturate GdN or DyN. From the divergence of the ZFC and FC curves it was determined that $T_{C}=27 \pm 3 \mathrm{~K}$.

Figure 7.2 shows magnetic field sweeps of a $\mathrm{SmN}$ sample at $15 \mathrm{~K}, 10 \mathrm{~K}$, and $2 \mathrm{~K}$. At each temperature the sweep was performed after cooling in a field of $6 \mathrm{~T}$ and after cooling in zero-field. In each case the data appear to be superimposed on a linear background. This is due to the paramagnetic response of the GaN capping layer, which is visible due to the small signal from the SmN [101]. Turning first to the ZFC data, a hysteresis loop is clearly visible at $15 \mathrm{~K}$ and saturates at $3 \mathrm{~T}$ with a coercive field of $0.9 \mathrm{~T}$. At $10 \mathrm{~K}$ the loop does not quite close - the saturation field is greater than $6 \mathrm{~T}$. This behaviour is exactly as expected when the applied field is insufficient to achieve reversal of the moments so that only minor loops are measured, 

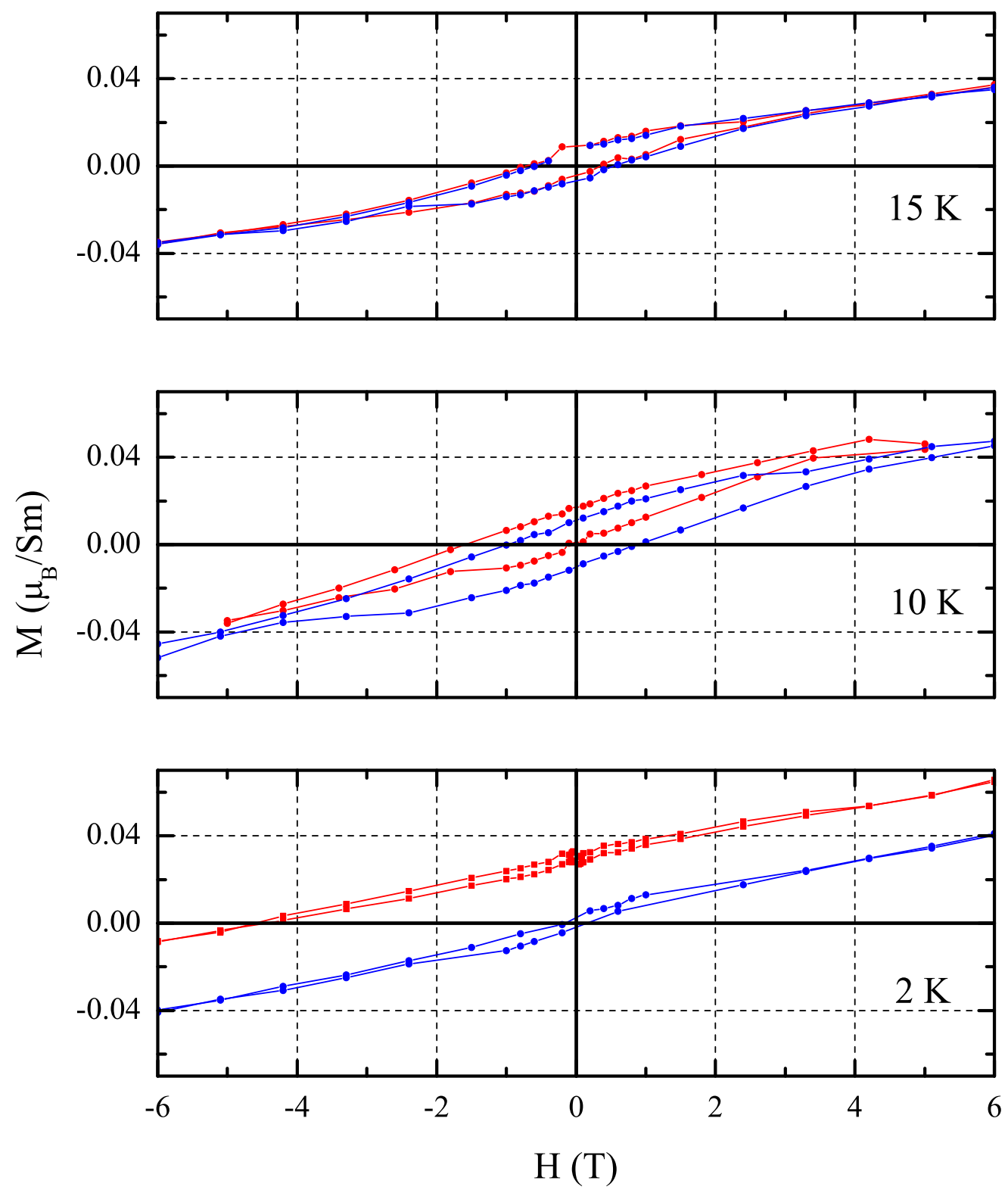

Figure 7.2: SmN magnetization versus applied field strength, measured after cooling in zero-field (blue circles) and after cooling in a field of $6 \mathrm{~T}$ (red circles). The measurement was repeated at $15 \mathrm{~K}, 10 \mathrm{~K}$, and $2 \mathrm{~K}$. 
signalling a magnetocrystalline anisotropy that grows at lower temperature. At $2 \mathrm{~K}$ the hysteresis has almost completely disappeared, implying that the magnetic field necessary to initiate reversal of the ferromagnetic domains is higher than 6 T. Even though a larger applied field is not available hysteretic behaviour can still be confirmed by the $6 \mathrm{~T}$ FC data, which prepares the film in a magnetized state. Comparing the FC and ZFC data, the loops are superimposed at $15 \mathrm{~K}$ but are offset at lower temperatures. This confirms that the coercive and saturation fields increase to more than $6 \mathrm{~T}$ at lower temperatures. By cooling the film in a $6 \mathrm{~T}$ field it is possible to prepare the film in an ordered state. The remnant magnetization at low temperature is measured to be $0.035 \pm 0.010 \mu_{B} / \mathrm{Sm}$ in agreement with the calculation of Larson et al., where the total moment per $\mathrm{SmN}$ is $0.029 \mu_{B} / \mathrm{Sm}$. This is in contrast to the Hund's rule ground state for the $\mathrm{Sm}^{3+}$ ion where the orbital and spin moments cancel completely.

A Curie-Weiss fit of the temperature dependent susceptibility data (not shown) fails. This is because although the first excited multiplet is too high in energy to be thermally populated, it is possible for a contribution to the paramagnetic moment to arise from a field induced admixture of the two multiplets. In this case a Van Vleck approach is necessary (see Equation 4.5). For $\mathrm{Sm}^{3+}$ the energy difference between the two lowest energy multiplets is $1500 \mathrm{~K}$. A fit to the susceptibility results in excellent agreement and a Curie temperature of $28 \pm 1 \mathrm{~K}$ ( $c f ., 27 \pm 3 \mathrm{~K}$ from the divergence of the ferromagnetic $\mathrm{FC}$ and ZFC curves). The effective moment is $\mu_{\mathrm{eff}}=0.45 \pm 0.10 \mu_{B} / \mathrm{Sm}$, less than the free-ion moment of $0.845 \mu_{B} / \mathrm{Sm}$. This can be understood by considering the $\mathrm{Sm}^{3+}$ ion in a cubic crystal field (as is the case for $\mathrm{Sm}$ in $\mathrm{SmN})$. In this case the $J=5 / 2$ state is split into $\Gamma_{7}$ and $\Gamma_{8}$ states, separated by roughly $225 \mathrm{~K}$. At $30 \mathrm{~K}$ only the $\Gamma_{7}$ state will be populated. In that case $\mu_{\text {eff }}=0.41 \mu_{B} / \mathrm{Sm}$, in agreement with the moment extracted from the fit to the experimental data.

To summarize, SmN has been shown to be a ferromagnetic semiconductor with a moment of $0.035 \pm 0.010 \mu_{B} / \mathrm{Sm}$ in the ferromagnetic ground state. A 


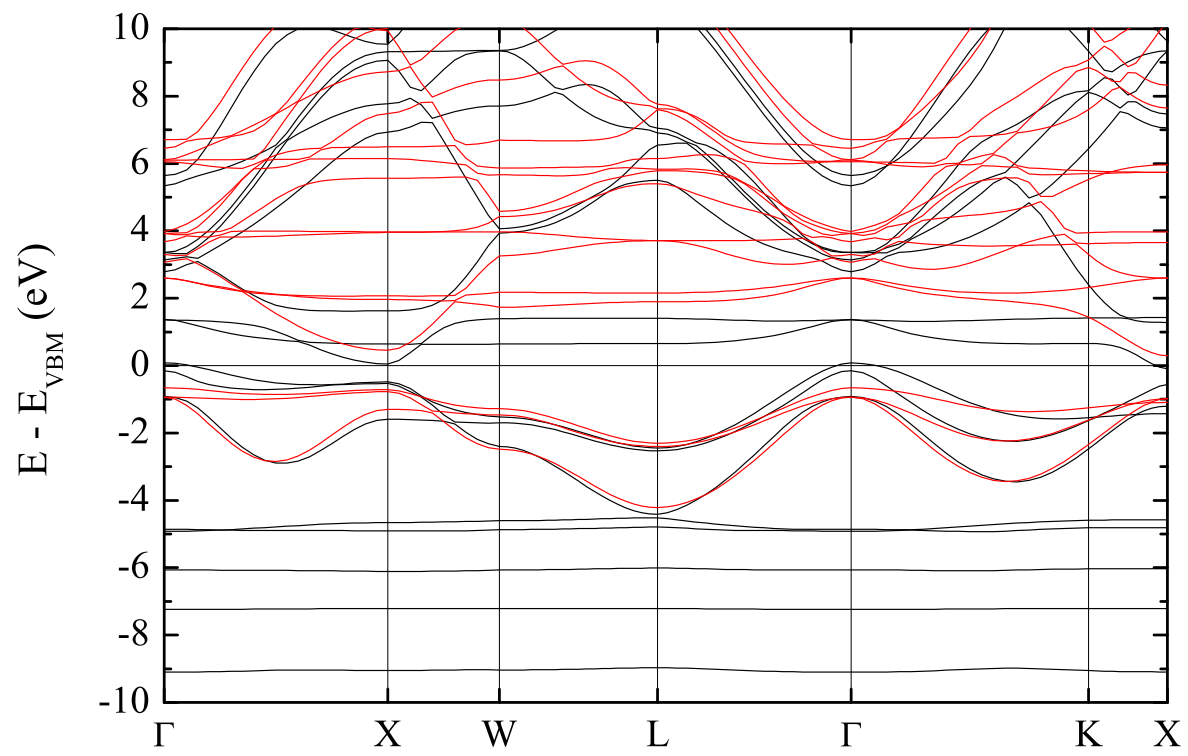

Figure 7.3: $\mathrm{SmN}$ band structure calculated with $U_{d}=6.4 \mathrm{eV}$ and $U_{f}=$ $8.22 \mathrm{eV}$. Majority- (black) and minority-spin (red) bands are isolated.

clear understanding of behaviour of the $\mathrm{Sm}^{3+}$ ion is important for fitting the paramagnetic response. It is shown that the cubic crystal field affects the ion. This is interesting in light of XAS measurements of the SmN M-edge, and recent theoretical results, which indicate that crystal field effects are small enough to not directly affect $4 f$ electrons in electronic band structure calculations [13]. Given that the ferromagnetic moment is in agreement with a calculation that attributes a significant moment of $0.135 \mu_{B}$ to each nitrogen ion $\left(c f ., 0.083 \mu_{B}\right.$ for GdN), XMCD experiments at the N K-edge, similar to those reported for $\mathrm{GdN}$ in Ref. [41], are desirable.

\subsection{Electronic Band Structure}

The band structures of $\mathrm{SmN}$ and $\mathrm{DyN}$ are shown in Figures 7.3 and 7.4. The calculations were performed using the same computational framework and parameters as Larson et al. [13]. Setting aside the $4 f$ states, the $\mathrm{SmN}$ and 


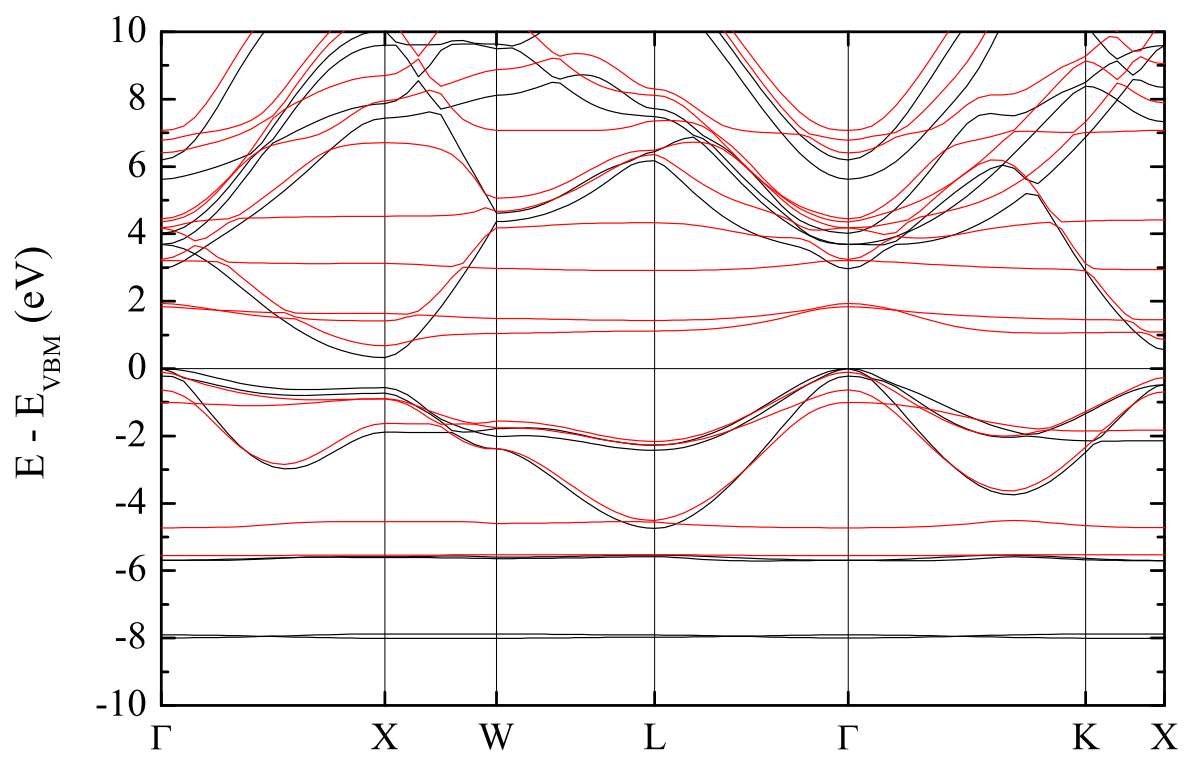

Figure 7.4: DyN band structure calculated with $U_{d}=6.4 \mathrm{eV}$ and $U_{f}=$ $9.32 \mathrm{eV}$. Majority- (black) and minority-spin (red) bands are isolated.

DyN band structures are in many ways similar to GdN. The VBM is at $\Gamma$ and the CBM is at X. Also, the majority- and minority-spin states are split so that the majority gap is smaller than the minority gap. These similarities are the underlying reason for the lack of major distinction between N K-edge XXS measurements of different ReNs.

The calculation uses $U_{d}=6.4 \mathrm{eV}$, which was determined by fitting the GdN calculation with previously measured optical data for GdN. With this value $\mathrm{SmN}$ is a semimetal with an overlap between the majority spin bands, but not the minority (i.e., it is a half-metal). The indirect majority and minority gaps are $-0.15 \mathrm{eV}$ and $1.09 \mathrm{eV}$ (avg. $=0.48 \mathrm{eV})$, respectively, and the average of the direct majority and minority gaps (the room temperature optical gap) is $0.81 \mathrm{eV}$. It was shown in Section 6.5 that $U_{d}=8.0 \mathrm{eV}$ is more correct for GdN. It is likely that calculating the $\mathrm{SmN}$ bandstructure with this larger $U_{d}$ value will shift the $5 d$ levels up enough that it is no longer a half-metal. DyN is a semiconductor with an indirect gap of $0.34 \mathrm{eV}$. The 
room temperature optical gap is predicted to be $1.17 \mathrm{eV}$. In terms of the gap sizes $\mathrm{SmN}<\mathrm{GdN}<$ DyN.

For both ReNs there is splitting within both the majority and the minority $4 f$ states, complicating the band structure picture relative to GdN. For $\mathrm{SmN}$ the five $4 f$ electrons split into four levels between about -4 and $-9 \mathrm{eV}$. The two unoccupied majority states sit about $1 \mathrm{eV}$ above the Fermi level, and the remaining unoccupied states are spread between there and roughly $7 \mathrm{eV}$. The unoccupied levels hybridize with the Sm $5 d$ and $6 s$ levels that make up the conduction band. DyN has nine $4 f$ electrons split into four distinct bands. The remaining unoccupied states are spread from roughly $2 \mathrm{eV}$ above the Fermi level to $8 \mathrm{eV}$. As with $\mathrm{SmN}$ there is some hybridization with the Dy $5 d$ and $6 s$ levels.

For both materials there are occupied $4 f$ levels very close to the bottom of the valence band. This means the degree of hybridization with the $\mathrm{N}$ $2 p$ levels will be higher than for GdN, where there is a significant energy difference between the $\mathrm{N} 2 p$ valence bands and the Gd $4 f$ levels. Indeed this is reflected in the strength of the hybrid peak seen in the $p$-projected PDOS shown in Figs 7.6 and 7.7, respectively. It may be that the hybrid peak is strong enough to observe in XES spectra. This is considered in the XES analysis to follow. As mentioned above the unoccupied $4 f$ bands hybridize quite strongly with the Re $5 d$ and $6 s$ bands. However, in the areas where the hybridization is strongest the band structure is most complicated. Indeed the hybridization is not readily observed in the unoccupied $p$-projected PDOS, meaning it will be difficult to extract information on the location of the unoccupied $4 f$ levels from XAS measurements.

It was noted by Larson et al. that allowing the $4 f$ levels to break the cubic symmetry of the ReN crystal is necessary for correct convergence of the $\mathrm{LSDA}+U$ calculation. For GdN, where the $4 f$ level has no net orbital moment, the ground state is not affected. On the other hand calculations for both SmN and DyN (where $L=5$ ) converge to a significantly lower energy if the cubic symmetry is not enforced. Because of this the three nitrogen $p$ 


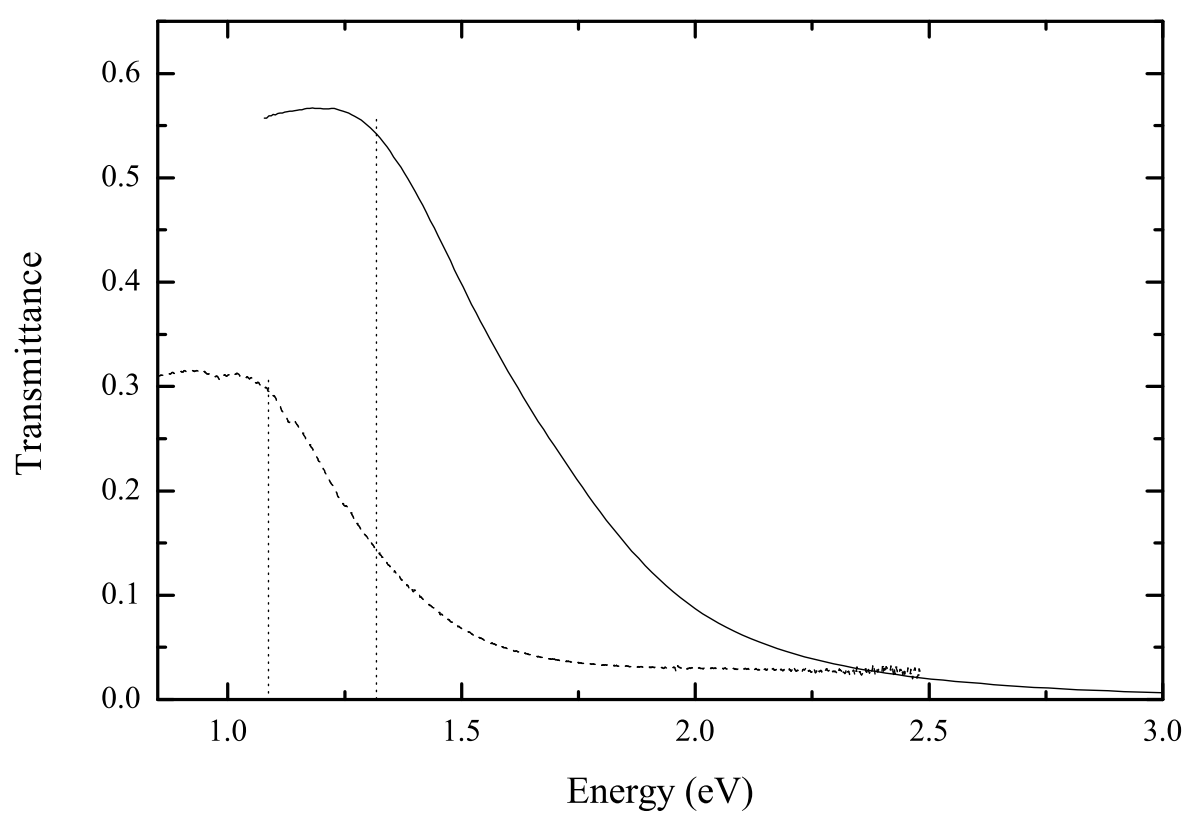

Figure 7.5: Optical transmittance data for $\mathrm{SmN}$ (dashed line) and DyN (solid line). The extrapolated gap positions are marked by dotted lines (see text).

bands (for each spin) which make up the VB are no longer degenerate at $\Gamma$. This has interesting implications for x-ray spectroscopy measurements where it may be possible to observe an angular anisotropy.

\subsection{Optical Spectroscopy}

Energy-dependent optical transmittance spectra were obtained from thin films grown by TED on sapphire and capped with GaN. The SmN and DyN data were obtained by FTIR and conventional optical spectroscopy, respectively. The spectra are shown in Figure 7.5. Both materials show evidence for an absorption edge above $1 \mathrm{eV}$ and the $\mathrm{SmN}$ sample, for which the lower side of the energy window was about $0.3 \mathrm{eV}$, shows strong interference fringes in the subgap region, but no evidence for a Drude response which would indicate a high concentration of free carriers. The low transmittance across the 
energy range is seen in more than one sample and is thought to be intrinsic to the film.

The intercepts of extrapolations of the subgap transmission and the inflection point on the absorption edge are used to estimate gap energies of $1.1 \pm 0.1 \mathrm{eV}$ for $\mathrm{SmN}$ and $1.3 \pm 0.1 \mathrm{eV}$ for DyN (indicated on Fig. 7.5 by dotted lines). Compared with the calculated room temperature optical gap (see above) the experimental SmN and DyN gaps are $0.29 \mathrm{eV}$ and $0.13 \mathrm{eV}$ larger, respectively. Increasing the $\mathrm{GdN} U_{d}$ value from $6.4 \mathrm{eV}$ to $8.0 \mathrm{eV}$ resulted in an increase of the room temperature optical gap from $0.98 \mathrm{eV}$ to $1.30 \mathrm{eV}$; it seems likely that an increase in the $U_{d}$ value for the $\mathrm{SmN}$ and DyN band structure calculations will improve agreement with experiment. Still, the optical data are very preliminary and more experimental data from high quality samples are needed.

\subsection{X-ray Spectroscopy}

Figures 7.6 and 7.7 show the N K-edge XES and XAS of SmN and DyN, respectively. The emission of both ReNs shows a single strong peak of $\mathrm{N} 2 p$ states near the VBM. A lower experimental resolution means the peaks are broader and show less structure than the equivalent GdN peak (Fig. 6.6), but asymmetry due to weight on the lower energy side is still visible. It is possible that the $2 p-4 f$ hybrids are contributing weight to the low energy side of the XES peak, but this is impossible to confirm without higher resolution data. The absorption spectra also show strong similarities, with a broad rise above the absorption edge followed by a series of peaks. It is noted that the first XAS peak is located at the same energy as the $\pi^{*}$ resonance of molecular nitrogen, as confirmed by measuring the XAS of a thin film of GaN known to contain $\mathrm{N}_{2}$ [102]. While the peak is too broad to assign solely to the molecular signal, a clear and sharp $\mathrm{N}_{2}$ peak develops after prolonged beam exposure. A similar effect was noted for GdN in Ref. [41], but not in the GdN from this thesis, suggesting it is of superior quality. It is noted that the 


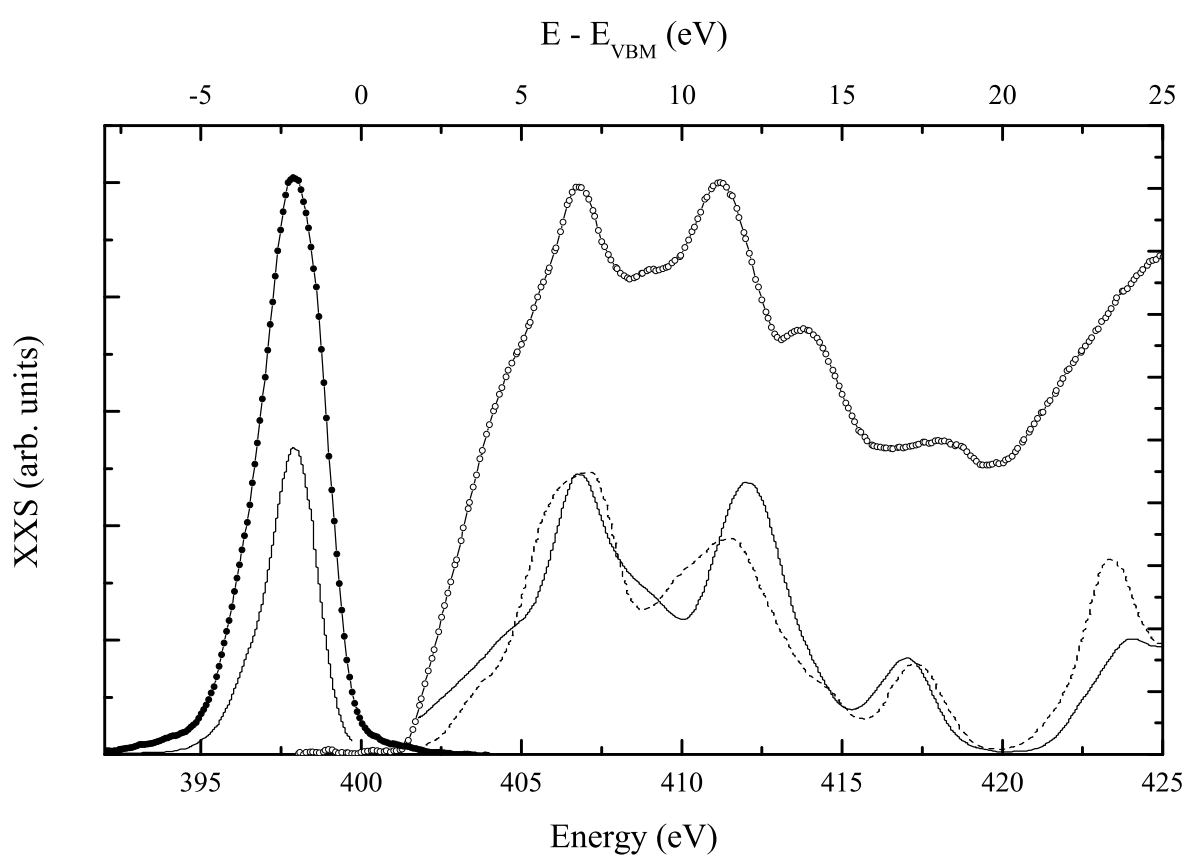

Figure 7.6: SmN N K-edge XES (solid circles) and XAS (empty circles). Also shown is the calculated PDOS (solid line) and PDOS from a core-hole calculation (dashed line).

undamaged peak agrees well with the calculated PDOS.

The $p$-projected PDOS, including both the ground state and core-hole spectra for the unoccupied states, are also shown in Figs. 7.6 and 7.7. This is in excellent agreement with the XES, with the asymmetry on the low energy side of the main peak well described. For the XAS the core-hole calculation provides a remarkably good fit, reproducing even the high-energy features in both $\mathrm{SmN}$ and DyN. It is noted that the core-hole calculation does not significantly alter the $p$-projected PDOS, except for the attenuation of the second peak in the XAS.

Although a core-hole will alter the XAS in unpredictable ways, as a rule of thumb any shift will be downward in energy since the core-hole reduces the screening of the ionic potential (see Section 4.4.1). As a result any gap between the occupied states (XES) and unoccupied state (XAS) will be less- 


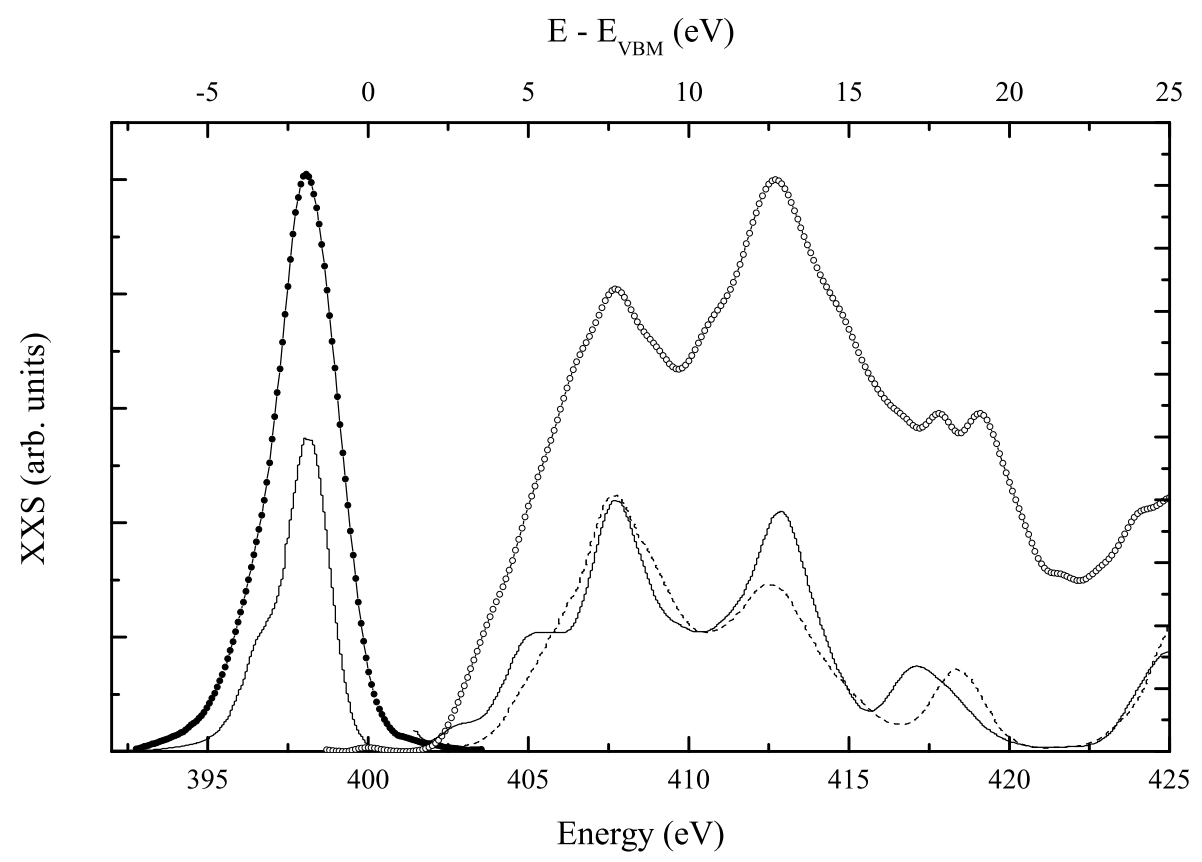

Figure 7.7: DyN N K-edge XES (solid circles) and XAS (empty circles). Also shown is the calculated PDOS (solid line) and PDOS from a core-hole calculation.

than or equal-to the true $p$-projected band gap. While this means it is impossible to extract an exact band gap, a lower limit on the gap can still be obtained. Here, the gap (measured by extrapolation) between the VBM and $\mathrm{CBM}$ is about $1.4 \mathrm{eV}$ and $1.0 \mathrm{eV}$ for $\mathrm{SmN}$ and $\mathrm{DyN}$, respectively. These numbers are similar to those extracted from the optical transmittance. Difficulties with measuring the true VBM and CBM in the XXS - experimental resolution and sensitivity may be too low to observe the weakly $p$-like CBM - make more quantitative statements impossible.

\subsection{Conclusions and Outlook}

The electronic structures of $\mathrm{SmN}$ and DyN were investigated in this chapter. Transport and optical transmission measurements indicate that both mate- 
rials are semiconductors, although the samples appear to be heavily doped. Magnetic measurements show that both $\mathrm{SmN}$ and DyN are ferromagnets and that $\mathrm{SmN}$ has a near-zero moment in the ferromagnetic state. The absorption edges extracted from the optical transmission imply that a larger $U_{d}$ value is needed in the LSDA $+U$ calculations. A comparison of the XXS with PDOS shows good agreement.

It has been shown that it is extremely difficult and perhaps impossible to locate the Re $4 f$ levels with $\mathrm{N} \mathrm{K}$-edge spectroscopy. It is very important to accurately determine the position of these, especially when split in the complicated manner of $\mathrm{SmN}$ and DyN. Future measurements of the $4 f$ levels by XPS are important although it is noted that XPS is not an orbitally resolved technique so the $\mathrm{N} 2 p$ and Re $4 f$ levels will interfere with each other.

The near-zero moment of SmN, not seen in a ferromagnetic semiconductor before, has great potential to be exploited in future spintronic devices; it would be useful in any spintronic device where a ferromagnetic material is required, but where stray fields are undesirable. For example, a spin resolving scanning tunneling microscope tip with a negligible fringe field would be ideal for measuring the magnetic properties of samples on the nanoscale [12]. Therefore, future work will likely focus on this material with an aim toward high quality film growth. 



\section{Chapter 8}

\section{X-ray Spectroscopy Across the ReN Series}

\subsection{Nitrogen K-edge}

To complement the GdN, SmN, and DyN XXS presented in previous chapters, N K-edge XXS was obtained for LuN and HfN. Both materials have a full $4 f$ shell so the majority- and minority-spin electrons are degenerate. In band structure calculations the full $4 f$ shell can be treated almost as a core level, removing the primary difficulty of including multiplet effects. Therefore, $\mathrm{LuN}$ is an excellent control when searching the $\mathrm{N}$ K-edge spectra for evidence of $2 p-4 f$ hybridization. Preliminary data are presented here for subsequent refinement of electronic band structure calculations.

Figure 8.1 shows the XES of the ReNs. Here the SmN and DyN spectra, which were calibrated differently to the other three spectra, have been arbitrarily shifted in energy so that the main peak matches with GdN. The dotted line indicates the Fermi level of HfN, which is evidently located roughly $3 \mathrm{eV}$ higher than the VBM of HfN and of the ReNs. Across the ReN series the XES are quite similar. LuN shows a slight difference in that both the main peak and the lower energy shoulder seem to be shifted downward in energy. This is also seen in the HfN XES and could be due to the reduced $4 f-5 d$ 


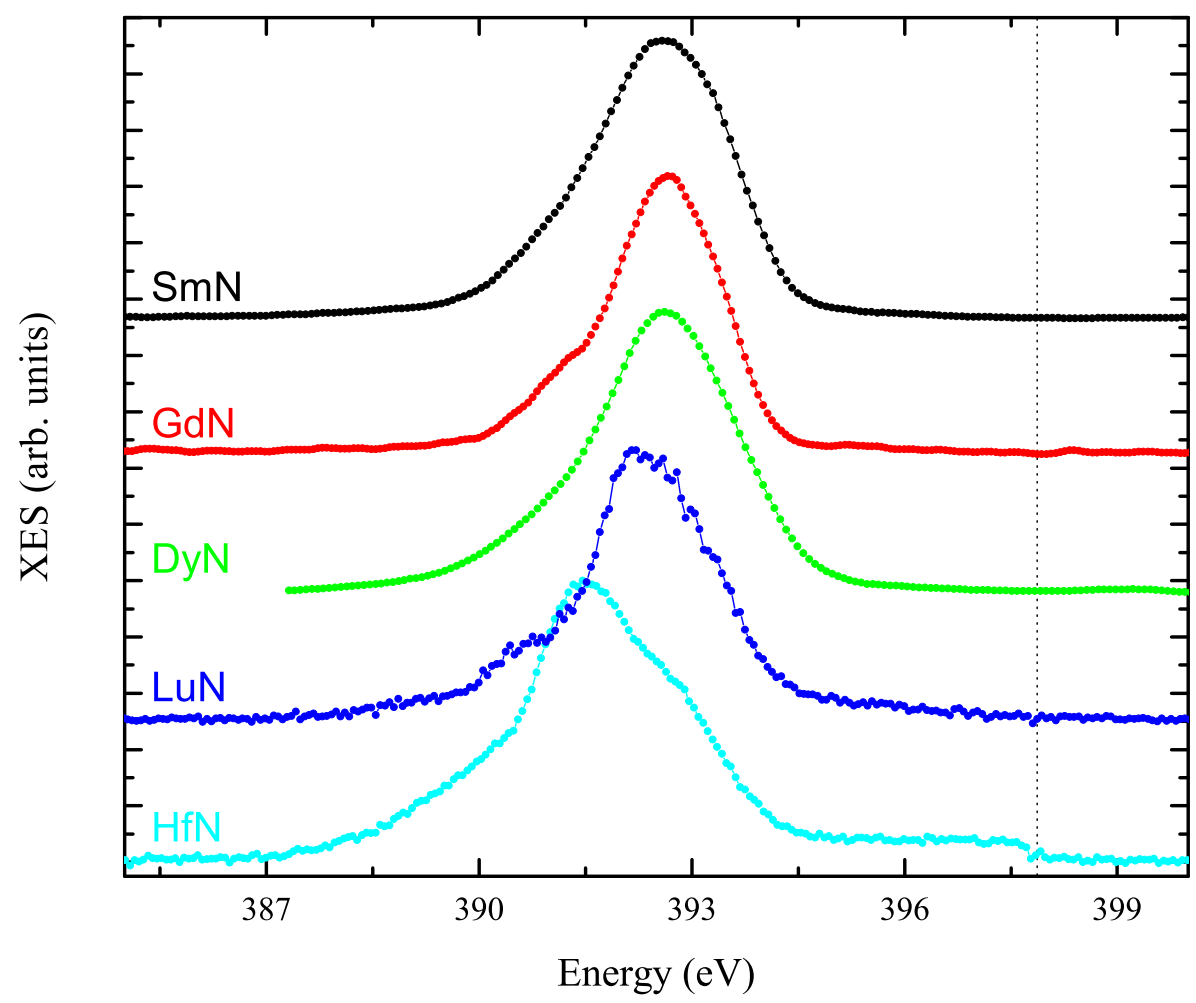

Figure 8.1: N K-edge XES of SmN, GdN, DyN, LuN and HfN.

repulsion which results from the fact that the full $4 f$ shell is more tightly bound.

The main SmN and DyN peaks show less structure than any of the other materials. This is possibly a result of lower experimental resolution or poorer crystal quality (the films were grown in situ at the beamline and thus no XRD was obtained), but may also be caused by the occupied $4 f$ levels which are predicted to lie at energies close to the N $2 p$ valence band. Further, it is pointed out in Ref. [13] that allowing the $4 f$ electrons to break the cubic symmetry breaks degeneracies in the $\mathrm{N}$ bands, as can be seen near $\Gamma$ in Fig 7.3. It could be that this reduced symmetry also broadens the partial density of states.

Figure 8.2 shows the XAS of the ReNs measured to date. Here again the spectra have all been arbitrarily shifted so that the first peaks are aligned. 


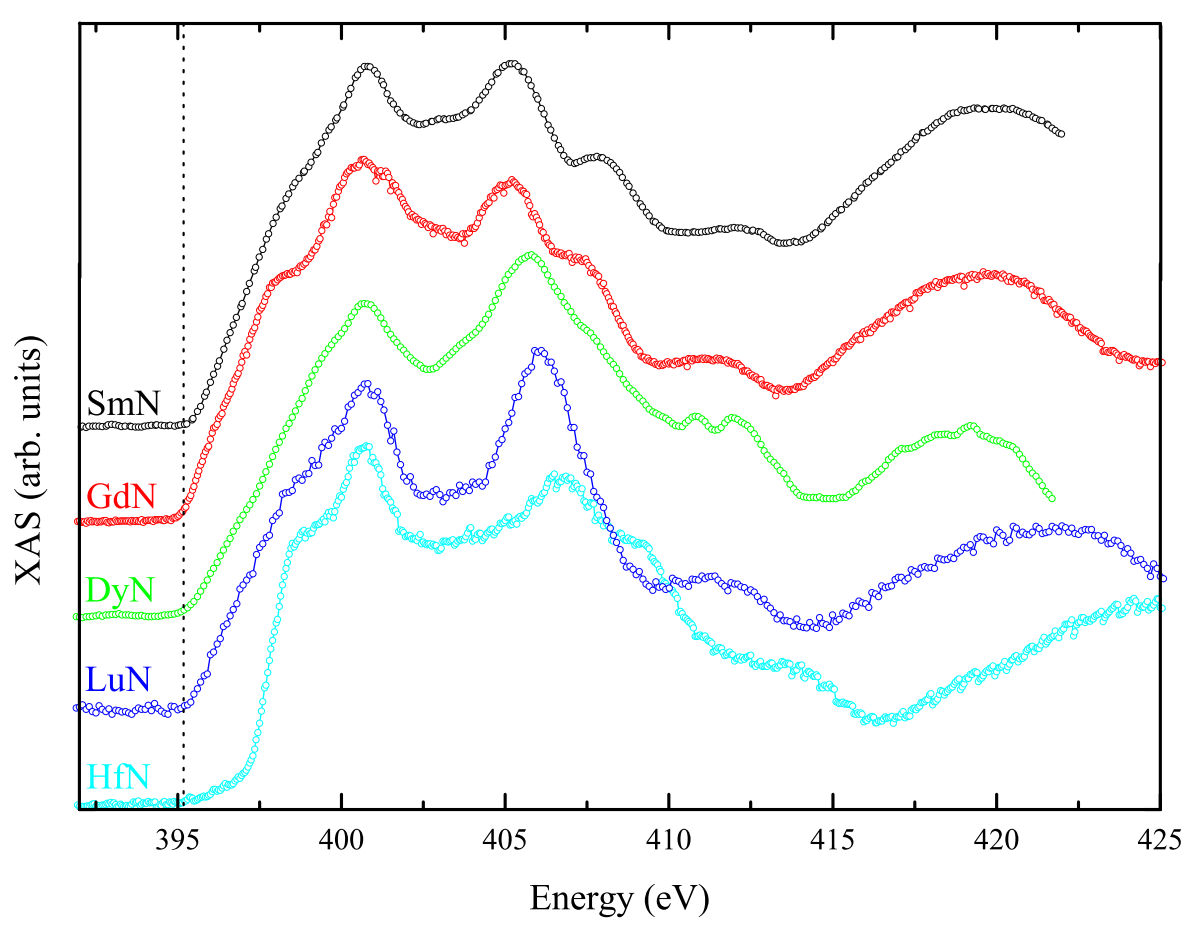

Figure 8.2: XAS of SmN, GdN, DyN, LuN and HfN.

The main XAS features are present in each case and the main differences seem to be systematic. The first two peaks, at roughly 401 and $405 \mathrm{eV}$, are assigned to the rare-earth $5 d \mathrm{t}_{2 g}$ and $\mathrm{e}_{g}$ orbitals, respectively, using the band assignments that were confirmed by the GdN RXES in Section 6.7.2. Relative to the first peak the second peak shifts to higher energy across the ReN series. The symmetry of the $t_{2 g}$ orbitals means that they point to the nitrogen nearest neighbours and can be thought of as $\sigma^{*}$ bonds, while the $\mathrm{e}_{g}$ bands point between the nitrogen nearest neighbours (toward the rare-earth nearest neighbours) and thus bond with nitrogen in a manner that can be considered a $\pi^{*}$ bond. The peak shift may be due to the differing symmetry of these two $5 d$ levels. In the minimum between the $\mathrm{t}_{2 g}$ and $\mathrm{e}_{g}$ peaks, at roughly $402.5 \mathrm{eV}$, a small feature is present in the $\mathrm{SmN}$, GdN, and DyN 


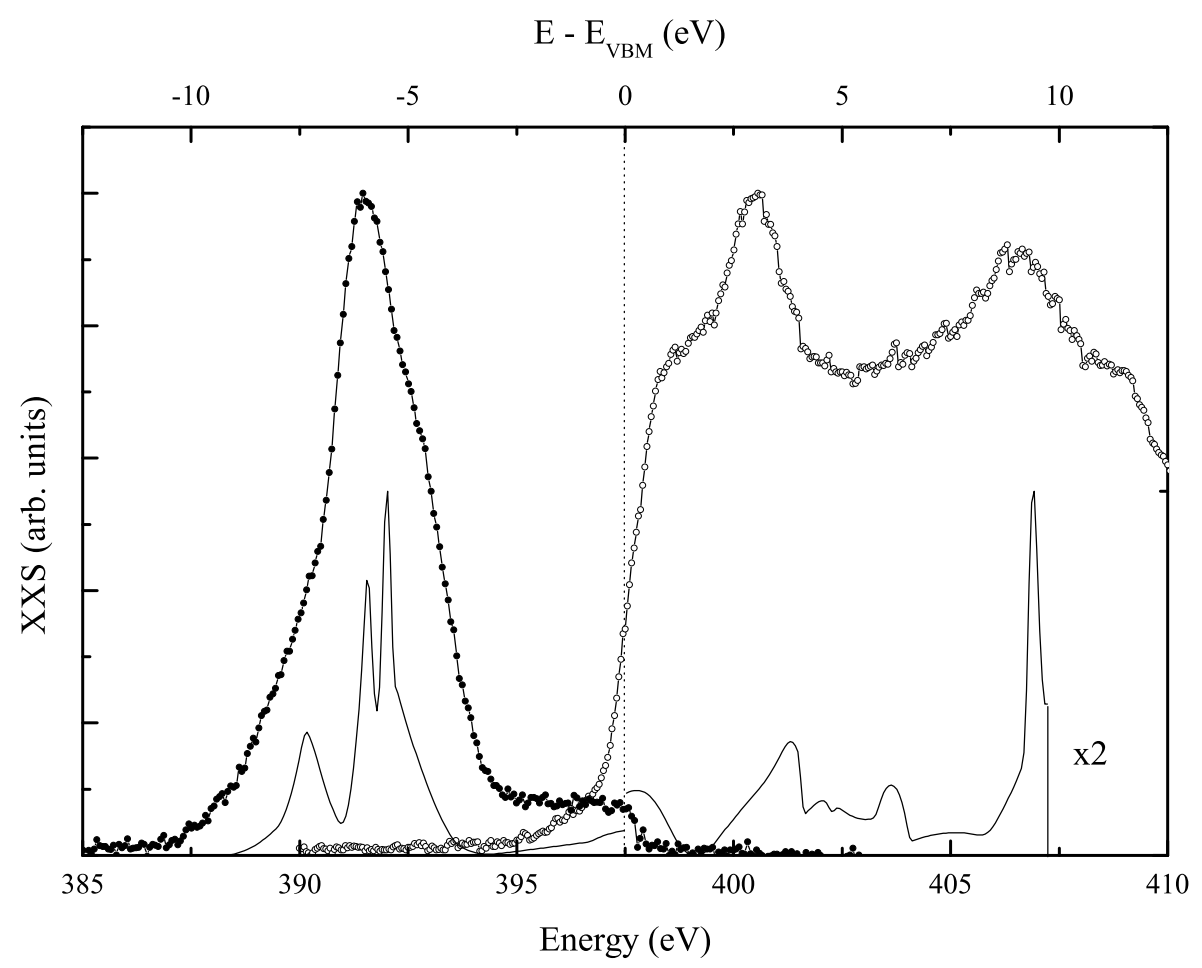

Figure 8.3: HfN N K-edge XES (solid circles) and XAS (empty circles). Also shown is the calculated PDOS (solid line). In this case $E-E_{\mathrm{VBM}}$ is the calculated position of the highest occupied electron state - the Fermi level.

samples that is not seen in $\mathrm{LuN}$ or HfN. This feature could be related to the $4 f$ levels but is too poorly resolved to say for certain.

Figure 8.3 shows the HfN XXS and calculated PDOS. The main difference between HfN and the ReNs is that the HfN Fermi level is clearly resolved in the XES by a sudden drop in the emission. This coincides with a sharp increase in the XAS and indicates that there is a density of states at the Fermi level. The calculated N K-edge PDOS agrees well with the main XES peak and places the highest occupied state well above the onset of the Hf $5 d$ band. As noted above, the HfN Fermi level lies roughly $3 \mathrm{eV}$ higher than the ReN VBM. This, and the similarity between the HfN and ReN crystal structures, means that it should be possible to create $\mathrm{Re}_{1-x} \mathrm{Hf}_{x} \mathrm{~N}$ alloys where 


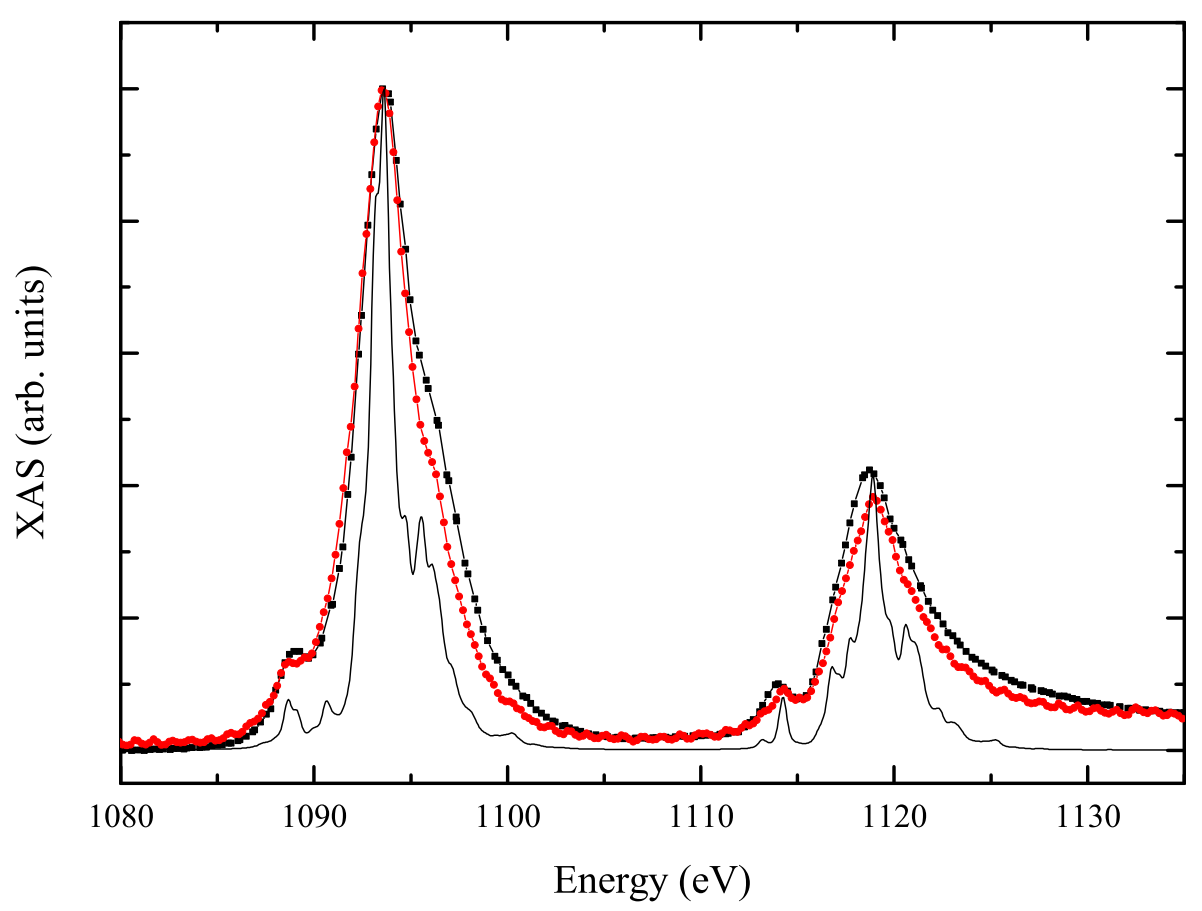

Figure 8.4: M-edge XAS of SmN (black squares), Sm (red circles) and an atomic multiplet calculation (line).

the extra Hf $5 d$ electron acts as an $n$-type dopant. For a semiconducting ReN it should then be possible to systematically increase the doping until the alloy is half-metallic.

\subsection{Rare-earth M-edge}

X-ray spectroscopy at the rare-earth $\mathrm{M}_{4,5}$ edges examines transitions between the $3 d$ core state and valence states and is used to directly probe the rareearth $4 f$ levels. Because the $4 f$ levels have a large angular momentum they tend to be located on the rare-earth site and intra-atomic effects tend to dominate. Indeed, significant progress toward describing the spectra presented in the following sections is made by considering entirely atomic effects, as detailed in Section 3.3. 
Figure 8.4 shows the XAS of SmN and Sm metal taken at the Sm Medge. It is obvious from the similarity between the two spectra that most of the M-edge structure is not unique to the nitride. The atomic multiplet calculation, which considers a $\mathrm{Sm}^{3+}$ ion in free space, shows that the XAS is in fact largely atomic-like in nature. The calculation, and those that follow, are based on the results of Thole et al. [47] and use the electrostatic and exchange parameters tabulated therein.

XAS was also obtained from EuN (see below), GdN (Section 6.7.3), DyN and $\operatorname{ErN}$ (not shown). In each case the agreement with the spectra calculated for the $\mathrm{Re}^{3+}$ ions is excellent, confirming that the atomic like nature of the uf levels holds across most of the ReN series.

Still, our aim is to investigate not just the rare-earth electronic structure but also the ReN electronic structure. It appears that if we are to extract information about this from the M-edge we need to look for subtle effects in higher resolution experiments. This will require a solid theoretical understanding of the theory of atomic multiplets and crystal field effects, subjects that are interesting and detailed in their own right.

\subsubsection{EuN and YbN charge state}

$\mathrm{Eu}$ and $\mathrm{Yb}$ have one less electron than the half-full and full $4 f$ shell, respectively. An interesting result from the LSDA $+U$ calculations of the ReNs by Larson et al. [13] is that the final converged band structures are sensitive to the initial state of the density matrix used to specify the $4 f$ electrons. In the calculation, densities corresponding to cubic symmetry and to atomic symmetry (Hund's rules) were considered. Across the series EuN and YbN are the two materials where the cubic symmetry initial state converges to a lower energy than the Hund's rule initial state. The cubic solution results in a $3+$ state for $\mathrm{Eu}$ and $\mathrm{Yb}$, while the Hund's rule solution converges to a $2+$ state, which does not seem to make sense for a ReN with 1:1 stoichiometry. That the two charge states are close in energy is perhaps unsurprising given that $\mathrm{EuO}$ and $\mathrm{Eu}_{2} \mathrm{O}_{3}$ both exist in nature. 


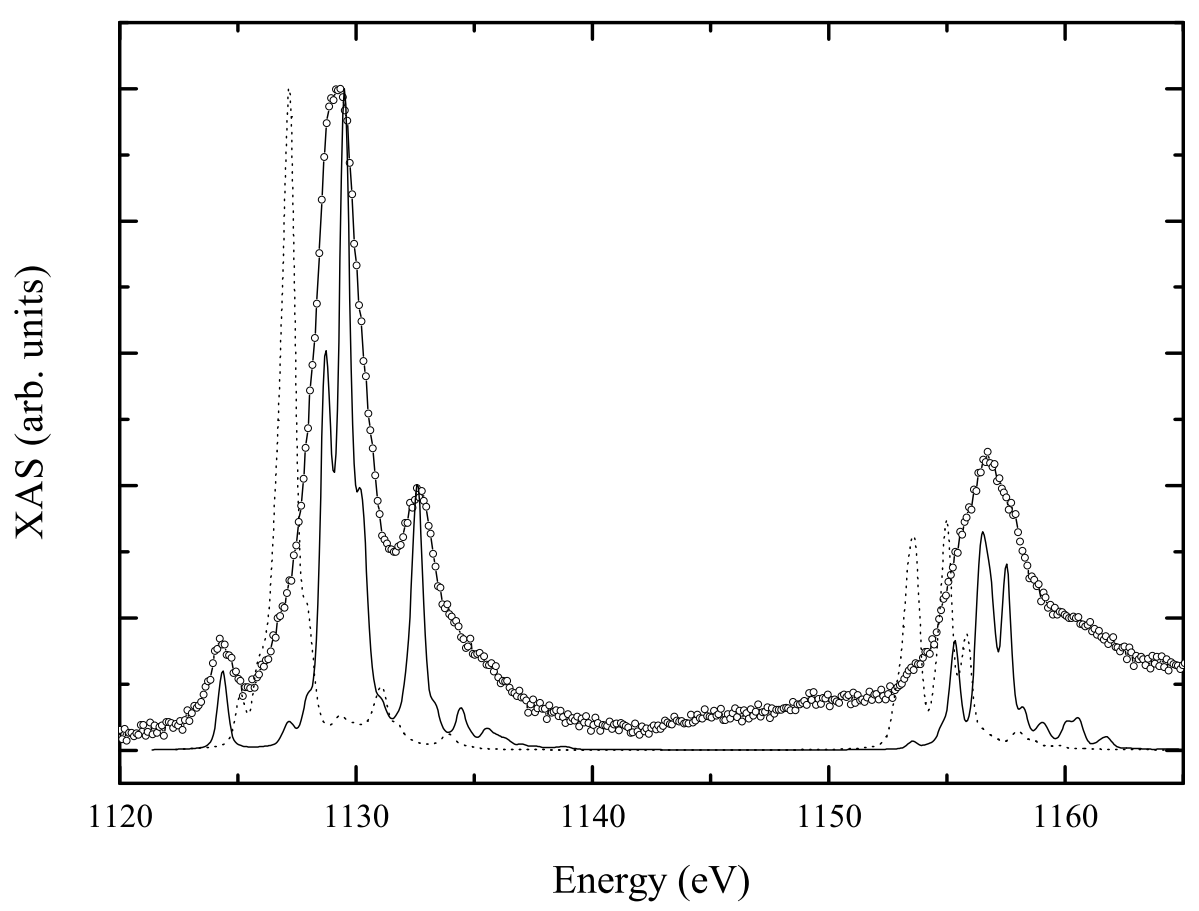

Figure 8.5: M-edge XAS of EuN (circles) compared with a calculation of the $\mathrm{Eu}^{3+}$ XAS (solid line) and the $\mathrm{Eu}^{2+}$ XAS (dashed line).

The theoretical picture is not clear, and experimental guidance is needed. There are some historical reports based on studies of the magnetism of the ReNs which suggest that $\mathrm{Yb}$ in $\mathrm{YbN}$ may be in the $2+$ state and that the $2+$ component of $\mathrm{Eu}$ in EuN increases with oxygen content [30, 24]. Perhaps the highest quality crystal and most comprehensive study of a ReN to date was reported by Degiorgi et al. in Ref. [25]. In their search for a heavy fermion system they produced stoichiometric single crystals of $\mathrm{YbN}$ by heating $\mathrm{Yb}$ metal shavings in the presence of nitrogen gas. They conclude that $\mathrm{YbN}$ is a self-compensated semimetal in the $3+$ state. Interestingly they locate the $2+$ state (14 electrons in the $4 f$ shell) just $0.2 \mathrm{eV}$ above the Fermi level. From their results it can be concluded that $\mathrm{Yb} 3+$ in $\mathrm{YbN}$ is the energetically favourable state, although it is very close to the $2+$ state, as Ref. [13] predicts.

EuN samples of the same quality as the $\mathrm{YbN}$ single crystals detailed 
above are not known to exist and sample growth has proved more difficult to achieve than for the other ReNs. Still, we have produced a few thin films of EuN by PLD where the lattice constant of the cubic crystal matches the expected value. The XRD spectrum of the best of these films is included in Fig. 5.2. A similar film was capped with $\sim 100 \mathrm{~nm}$ of $\mathrm{Cr}$ to allow transport to the synchrotron for XAS measurements.

Figure 8.5 shows the Eu M-edge XAS of this sample. Also included are the results of multiplet calculations of the Eu M-edge for $\mathrm{Eu}^{2+}$ and $\mathrm{Eu}^{3+}$. The calculated spectra have been normalized and rigidly shifted so that the main features match with experiment. It is noted that while the rigid shift is allowed, it must be the same for both calculations; the position of the $2+$ spectrum is fixed relative to the $3+$ spectrum. Qualitatively the $\mathrm{Eu}^{3+}$ calculation agrees well with experiment. A fit of linear combinations of the normalized calculated spectra to experiment indicate that less than $1 \% \mathrm{Eu}^{2+}$ exists in the sample. In actual fact the $2+$ ion is calculated to have a significantly larger absorption cross section so the true percentage of $\mathrm{Eu}^{2+}$ is probably somewhat lower. Ref. [47] shows that Eu metal exists in the 2+ state, therefore the $\mathrm{Eu}$ in the EuN sample is not metallic. It is noted that the native oxide $\left(\mathrm{Eu}_{2} \mathrm{O}_{3}\right)$ would give the same signal as above. On the other hand the XRD would be different.

It seems possible then that both $\mathrm{Eu}$ in $\mathrm{EuN}$ and $\mathrm{Yb}$ in $\mathrm{YbN}$ exist in the $3+$ charge state. This is in impressive agreement with the calculation by Larson et al. That they converge to the $3+$ result only when the initial $4 f$ state has the cubic symmetry implies that crystal field effects may be more important in these two ReNs than in the rest of the series.

\subsection{Conclusion}

$\mathrm{N}$ K-edge XXS indicates that the local bonding environment around the $\mathrm{N}$ sites is largely devoid of rare-earth $4 f$ character, while rare-earth M-edge XAS indicates that the majority of the $4 f$ orbitals are located on the rare- 
earth site with wavefunctions that are very similar to those calculated for the $\mathrm{Re}^{3+}$ ion. In fact the M-edge XAS also provides evidence that the rare-earth $4 f$ orbitals in ReNs behave much as the rare-earth $4 f$ orbitals in rare-earth metals.

HfN is shown experimentally to have a much higher Fermi level than the other ReNs, implying that alloys could be created to systematically control the Fermi level and create a half-metal. Eu M-edge XAS of EuN hints that the $\mathrm{Eu}$ in $\mathrm{EuN}$ is in the $3+$ state, answering a key theoretical question. 



\section{Chapter 9}

\section{Band Structure of $\mathrm{ZnO}$ from Resonant X-ray Emission Spectroscopy}

\subsection{Motivation}

Zinc oxide is a wide-band gap post transition-metal oxide semiconductor with significant potential for use in for future opto-electronic devices. Among its many notable properties it has one of the largest known exciton energies for a crystalline material $(\sim 60 \mathrm{meV})$, making it a highly efficient light emitter [103]. Similarly to the ReNs, it is a system where a pure theoretic understanding of the electronic structure is complicated by the close proximity of localized electrons to the Fermi level - the Zn $3 d$ states lie about $8 \mathrm{eV}$ below the Fermi level, roughly the same energy as the filled $4 f$ states in GdN.

The current necessity for capping layers in the ReNs make electronic structure investigations by the photoemission techniques of XPS and ARPES problematic. $\mathrm{ZnO}$ is plagued by similar problems, but for a different reason - it is susceptible to charging effects which distort photoemission [74]. Therefore, for both the ReNs and $\mathrm{ZnO}$, it is important to develop photonin-photon-out techniques that enable electronic structure calculation, like 
RXES, to their full potential. Compared to the ReNs, significantly more work has been devoted to $\mathrm{ZnO}$ and high quality samples are readily obtained. This means that work into the theoretical and experimental development of RXES as a characterization technique can be more readily begun.

A RXES study into the bulk electronic structure of $\mathrm{ZnO}$ is also important for a number of fundamental technical reasons. For example, a number of technical problems may be related to interface effects [104], which dominate photoemission experiments. Additionally, stable p-type doping is essential and reliable integration with conventional silicon based devices is important. These problems can only be fully addressed with a complete understanding of the bulk electronic structure.

Given the interest, potential utility of $\mathrm{ZnO}$, and availability of quality samples, there are surprisingly few descriptions of the band structure in the literature, even when considering the experimental difficulties. A small number of ARPES studies have been reported [104, 105, 106], yielding some agreement with DFT-based calculations and providing information on surface and defect states. However, several outstanding issues remain. For example, photoemission measurements have variously located the fully occupied Zn $3 d$ semi-core levels at energies ranging from 7.5 to $8.8 \mathrm{eV}$ below the Fermi level [104, 105, 106, 107, 108, 109]. There are also reports of XAS and XES measurements of the O $2 p$ PDOS of nano-structured ZnO $[110,111]$ and its anisotropy in the conduction band [112], but none of the high quality single crystal material we will present here.

In this work high resolution XES and XAS data are used to determine the bulk occupied and unoccupied O p-projected PDOS of single crystal ZnO. The Zn $3 d$ level is clearly resolved in the K-edge XES due to Zn $3 d-\mathrm{O} 2 p$ hybridization, and its energy measured. Additionally, the $\mathbf{k}$-selection rules of RXES are exploited to map the anisotropic valence band of single crystal $\mathrm{ZnO}$. This work enhanced the utility of these techniques when applied to GdN in Section 6.7.2. 


\subsection{Experimental Details}

\subsubsection{Film growth}

The sample, a $500 \mathrm{~nm} \mathrm{ZnO}$ epilayer grown on epi-ready (0001) sapphire substrates by plasma-assisted MBE, was obtained from S. Durbin et al. of Canterbury University. Growth details are described in Ref. [113]. As the sample was part of a separate study on doping, approximately $0.01 \% \mathrm{Ag}$ was incorporated during growth, resulting in a layer averaged electron concentration of $7.3 \times 10^{16} \mathrm{~cm}^{-3}$. The high crystalline quality of the film was confirmed by RBS under the channeling condition [114], a streaky RHEED pattern, and by low temperature photoluminescence, which shows donor bound exciton peaks are dominant with up to three LO phonon replicas.

\subsubsection{X-ray spectroscopy}

The x-ray spectroscopy was performed on the undulator beamline X1B at the National Synchrotron Light Source at Brookhaven National Laboratory, which is equipped with a spherical grating monochromator and a Nordgrentype emission spectrometer. The energy resolution over the O K-edge was approximately $0.20 \mathrm{eV}$ and $0.37 \mathrm{eV}$ for the XAS and RXES respectively. XAS was recorded in TEY mode. The photon energy was calibrated using the $\mathrm{O}$ K-edge and $\mathrm{Ti}$ L-edges of rutile $\mathrm{TiO}_{2}$ measured during the experiment. The XES was calibrated with the 2 nd order L-edge of $\mathrm{Zn}$ from both a calibration metal sample and the $\mathrm{ZnO}$ sample.

Due to its wurtzite structure, the $\mathrm{ZnO} p$-projected PDOS is strongly anisotropic [112]. To measure the anisotropy XAS and RXES were recorded for light incident at $20^{\circ}$ and $70^{\circ}$ relative to the sample normal, referred to as normal and grazing geometry, respectively. Dipole selection rules mean that XAS recorded in the normal (grazing) geometry are dominated by the contributions from $\mathrm{O} 2 p_{x y}\left(2 p_{z}\right)$ orbitals (see inset, Fig. 9.1). The opposite is true for RXES as the emission spectrometer is oriented perpendicular to 
the incident light: $\mathrm{x}$-rays incident normal (grazing) to the sample result in grazing (normal) RXES. Thus $p_{x y}$ RXES is analysed with $p_{z}$ XAS and vice versa. This correspondence has been observed for wurtzite GaN by Strocov et al. [97].

\subsection{Theoretical Details}

Electronic structure calculations were obtained from F. Bechstedt et al. of Friedrich-Schiller-Universität. They were performed within the hybrid DFT (HDFT) framework using the HSE03 functional for exchange and correlation, as described in Ref. [115]. Quasiparticle effects were taken into account by a subsequent $G W$ correction of the HSE03 eigenvalues using many body perturbation theory [116]. In the $G W$ calculations, the Coulomb potential was fully screened using the random phase approximation dielectric function based on the HSE03 eigenvalues and functions [117]. It has been shown that the $a b$ initio combination of HDFT and $G W$ calculations gives excellent values for the fundamental gaps and $d$-band positions of many semiconductors [118].

The XAS and XES are compared to PDOS obtained from the fully valid $G W$ calculation. However, because obtaining a high-resolution k-resolved $G W$ band structure is too computationally intensive, RXES data are compared to a scaled version of the HDFT band structure. The validity of this approach is verified in Section 9.4.2.

\subsection{Results}

\subsubsection{Partial density of states}

Figure 9.1 shows the O K-edge XAS and XES, taken well above threshold. Strong linear dichroism associated with the anisotropy in the unoccupied states is seen in the XAS, especially in the first $10 \mathrm{eV}$ above the conduction 


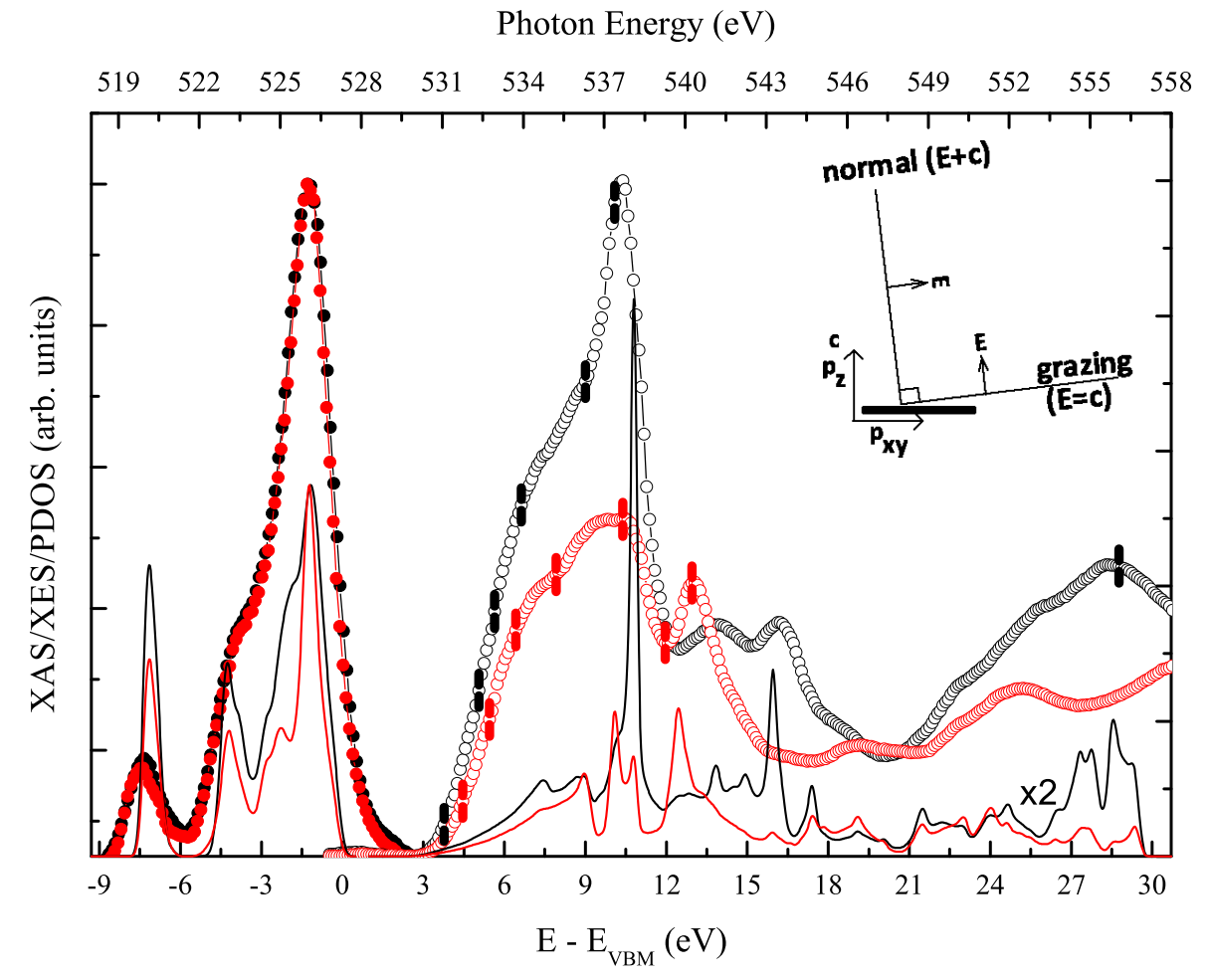

Figure 9.1: $p_{x y}$ (black) and $p_{z}$ (red) orbital contributions to XES (closed symbols) and XAS (open symbols) compared to PDOS from the $G W$ calculation (lines). Bars indicate RXES excitation energies.

band minimum where the $p_{x y}$ contribution is considerably stronger than the $p_{z}$ contribution. The XES is comprised of $\mathrm{O} 2 p$ states near the valence band maximum, along with a clear peak corresponding to $\mathrm{O} 2 p$ states hybridized with Zn $3 d$ orbitals at $519.9 \mathrm{eV}$.

These spectra are compared with PDOS obtained from the $G W$ calculation. The PDOS have been convolved using gaussians with widths of $0.37 \mathrm{eV}$ and $0.20 \mathrm{eV}$ for the occupied and unoccupied states respectively. This is an estimate of the intrinsic instrumental resolution of the experiment; no lifetime effects have been considered. The PDOS have been aligned so that the main valence band peak matches with experiment. This yields excellent agreement with the entire XES, reproducing the main valence band features 
and accurately locating the $3 d$ hybrid. The weak anisotropy in the PDOS, combined with the lower resolution of the XES, explains the lack of contrast between $p_{x y}$ and $p_{z} \mathrm{XES}$.

The final state in XAS includes a core-hole that is not included in the calculation, and which prevents absolute comparison between the energy scales of the XAS and XES [68]. Therefore, to compare to XAS, the theoretical unoccupied PDOS has been rigidly shifted by an additional $-1.0 \mathrm{eV}$, providing an estimate of the core-hole binding energy. With this adjustment the experimental agreement is excellent in terms of both the main peak locations and intensities, and the orbital anisotropy.

\subsubsection{Accuracy of theoretical approach}

The success of the $G W$ calculation is highlighted by comparison with the HDFT result. We calculate a band gap of $3.2 \mathrm{eV}$, compared to $2.1 \mathrm{eV}$ obtained from the HDFT calculation (and $3.4 \mathrm{eV}$ experimentally [104]). The valence band PDOS is also greatly improved: Figure 9.2 (a) shows the total O $2 p$-projected occupied PDOS calculated using HDFT, and the PDOS with the $G W$ correction applied. With $G W$ the Zn $3 d-\mathrm{O} 2 p$ hybrid peak is shifted from $-6.2 \mathrm{eV}$ to $-7.1 \mathrm{eV}$, in much closer agreement with the experimental peak at $-7.4 \mathrm{eV}$, correcting a key flaw of standard DFT calculations [118]. With the Zn $3 d$ states located lower in energy the rest of the valence band is widened towards lower energies due to reduced $p$ - $d$ repulsion. We note that scaling the HDFT energy axis by a factor of 1.1 (dashed line) is enough to obtain relatively close agreement with the $G W$ calculation for the occupied states.

The accuracy of this approach has been confirmed by calculating the computationally intensive $G W$ band structure at selected points in k-space, as shown in Figure 9.2 (b). Also shown is the band structure of the HDFT calculation with the scaling factor applied and with an additional upward shift of $0.5 \mathrm{eV}$ to the conduction bands. It can be seen that these post hoc alterations to the HDFT band structure result in an agreement with the $G W$ 
Photon Energy (eV)

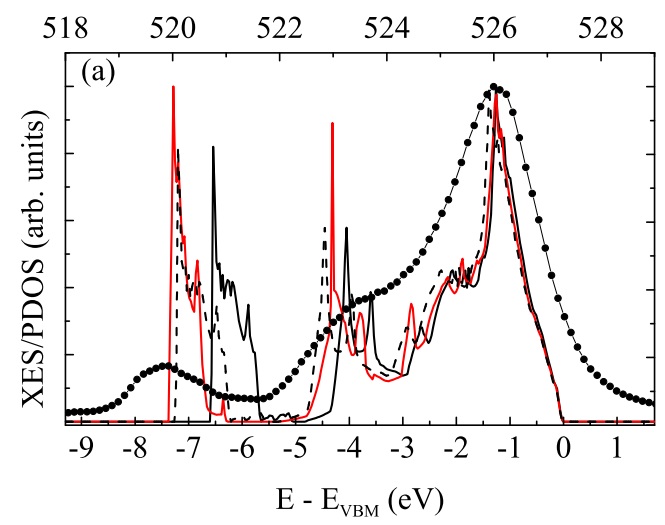

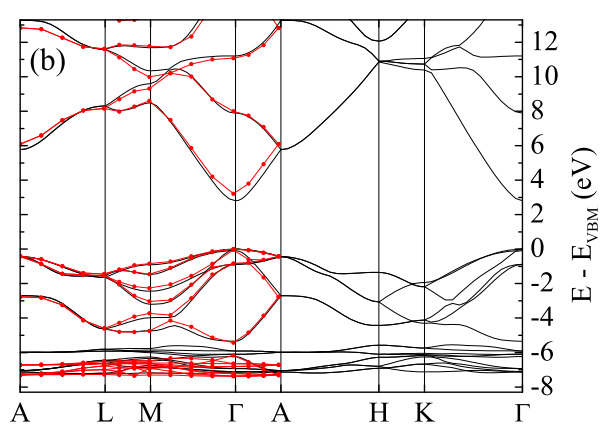

Figure 9.2: Comparison between $G W$ (red lines) and HDFT (black lines) calculations. (a) shows the $\mathrm{O} p$-projected PDOS of the occupied states. The $G W$ calculation more accurately positions the Zn $3 d-\mathrm{O} 2 p$ hybrid when compared with experiment (closed symbols). Multiplying the HDFT energy scale by a factor of 1.1 results in improved agreement (dashed line). compares the band structures obtained from the calculation. The HDFT energy axis has been scaled by a factor of 1.1 and the unoccupied bands have been shifted upward by $0.5 \mathrm{eV}$.

band structure that is accurate enough for our analytical purposes.

\subsubsection{Band structure dispersion from RXES}

Figure 9.3 shows the band structure obtained from the HDFT calculation, with the above corrections applied. The $p_{x y}$ and $p_{z}$ orbital contributions to the eigenvalues are plotted independently; error bars represent the relative weight of the contribution. To simplify RXES analysis, the $p_{x y}\left(p_{z}\right)$ unoccupied states are plotted with $p_{z}\left(p_{x y}\right)$ occupied states (see above). RXES can be viewed as a scattering process where the final state includes an electron in the conduction band and a hole in the valence band, but no core-hole. So, unlike Fig. 9.1 and Fig. 9.2 (b), no core-hole correction is applied to the conduction band. 

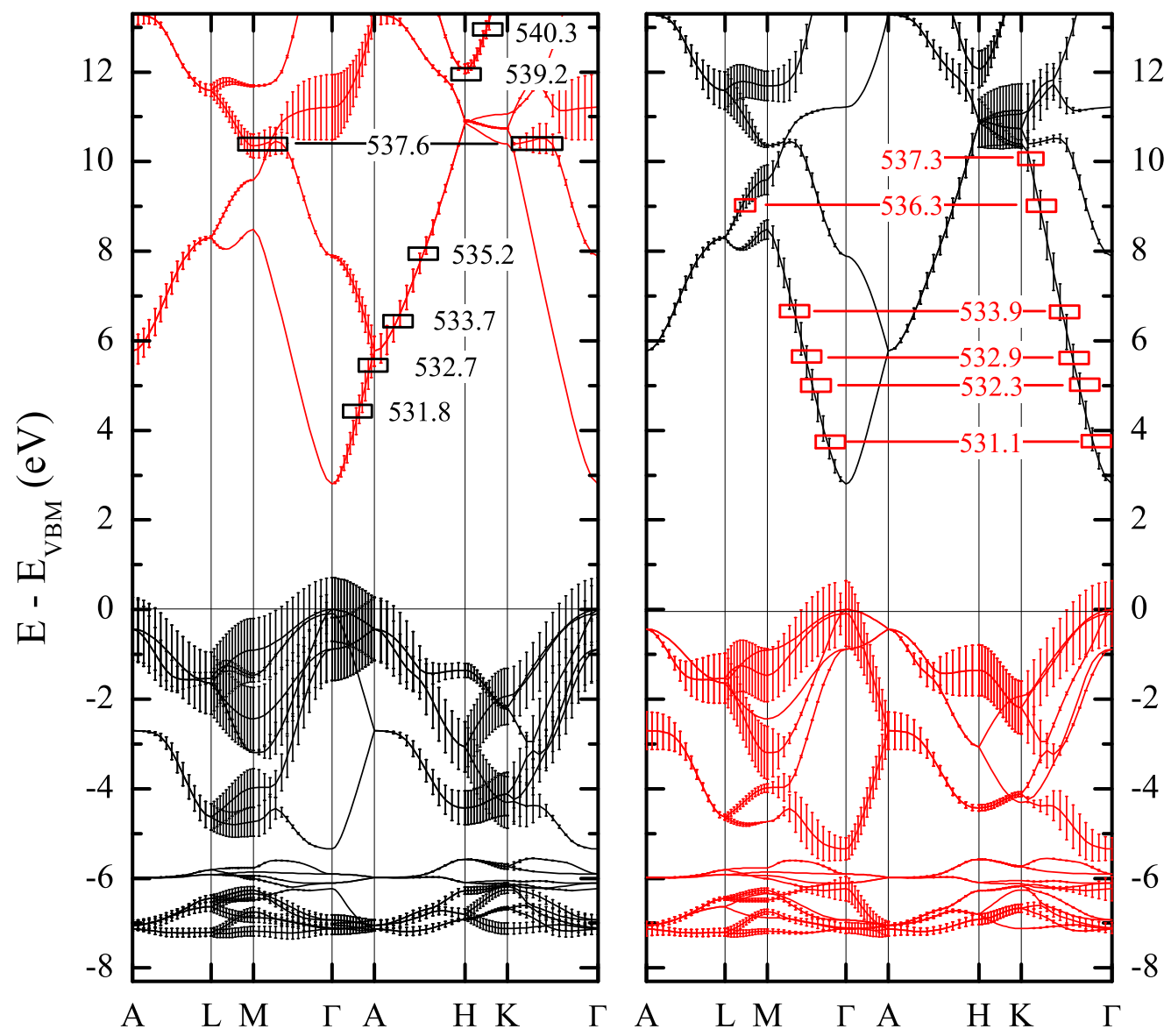

Figure 9.3: HDFT band structure. Calculation is orbitally resolved into $\mathrm{p}_{x y}$ (black) and $\mathrm{p}_{z}$ (red) contributions. Vertical bars represent strength of contribution. The energy axis has been scaled for better agreement with $G W$ theory (see Fig. 9.2). Labels indicate RXES excitation energies. 
As expected, the band structure is that of a direct gap semiconductor. The strongly dispersing band that forms the $\mathrm{CBM}$ at $\Gamma$ is also anisotropic: only $p_{x y}$ states are found along $\Gamma-\mathrm{M}$ and $\Gamma-\mathrm{K}$, while only $p_{z}$ states are found along $\Gamma-\mathrm{A}-\mathrm{H}$. This combination of strong dispersion and orbital selectivity make $\mathrm{ZnO}$ especially suitable for band mapping with RXES. For weakly correlated systems, and excitation energies close to the absorption edge, resonant effects contribute to XES, adding a k-conserving coherent contribution to the scattering, as described in Section 4.4.3. The crystal anisotropy, combined with orbital selection rules, adds further k-selectivity to the RXES. For ZnO, the use of these properties in concert allows valence band dispersion to be measured across a wide portion of the Brillouin zone.

RXES was taken over a range of excitation energies, indicated by vertical bars on the XAS of Fig. 9.1, Figs. 9.5 and 9.6 and the horizontal bars on Fig. 9.3. The coherent fractions of the RXES, extracted using standard techniques (see Section 4.4 .3 and Ref. [72]), ranged from 0.45 to 0.6 and are plotted in Figure 9.4.

Figures 9.5 and 9.6 show the initial technique used for analyzing the RXES. Figure 9.5 shows RXES (XAS) measured in the orientation that accentuates the $p_{x y}\left(p_{z}\right)$ contribution. As noted above, the geometry of the experiment forces this comparison. Figure 9.6 shows the opposite - RXES (XAS) measured to accentuate the $p_{z}\left(p_{x y}\right)$ contribution. Also shown in the top half of each figure are relevant parts of the calculated band structure. To allow comparison between experiment and theory the axes have been rigidly shifted as in Fig. 9.1. The arrow overlaid on the unoccupied states indicates that the RXES were taken at successively decreasing excitation energies. This shows how the portion of $\mathbf{k}$-space into which the core electron is being excited changes with incident photon energy. By making the assumption that momentum is conserved in the coherent RXES process it is possible to label the complementary parts of the occupied states from which the emission will preferentially originate from. For example, a $532.3 \mathrm{eV}$ photon $(5.0 \mathrm{eV}$ on the $E-E_{\mathrm{VBM}}$ energy scale) will generate resonant emission from the A-point 


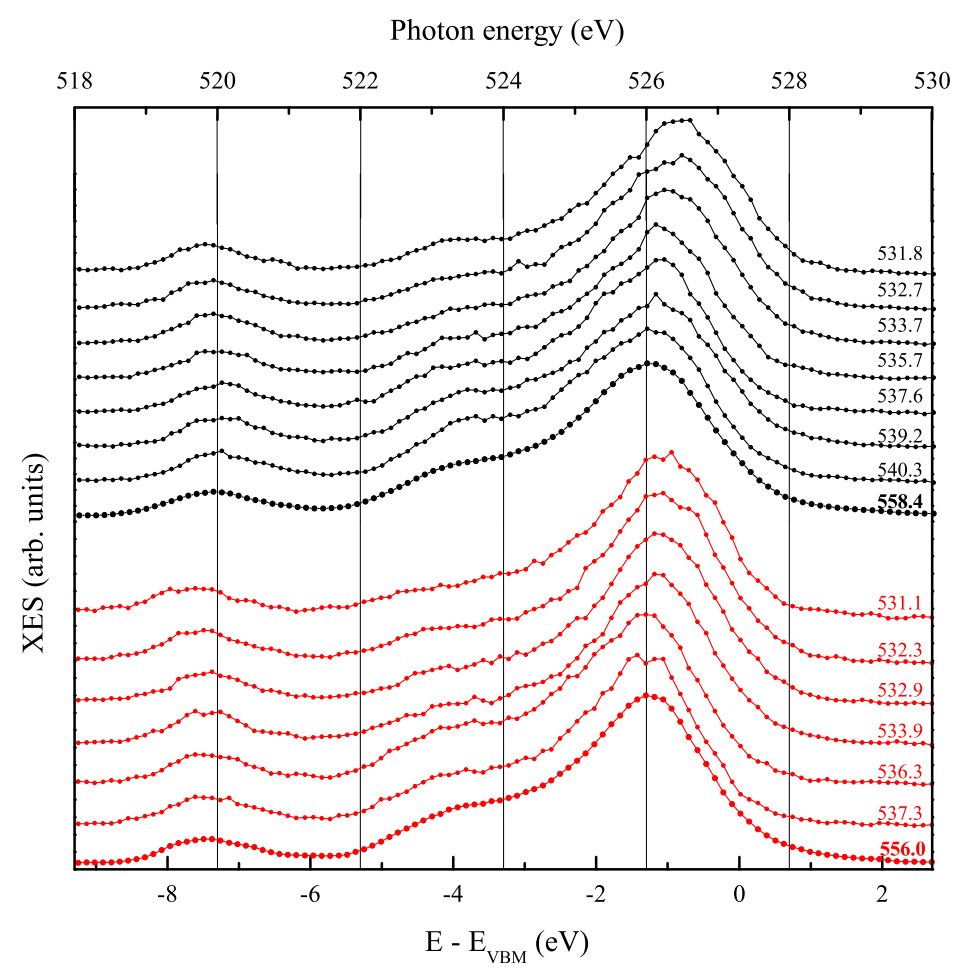

Figure 9.4: Normalized coherent RXES showing $p_{x y}$ (black) and $\mathrm{p}_{z}$ (red) data. Labels indicate RXES excitation energies.

(K-point) of the Brillouin zone of the $p_{x y}\left(p_{z}\right)$ states, as seen in Figs. 9.5 (9.6). These trends can be clearly seen in the RXES, indicating that the theoretical framework is consistent with experiment.

An alternative and more quantitative method of analysis involves the use of image maps. Using the coherent RXES we construct Figure 9.7, which shows normalized intensity maps of the coherent fraction of each spectrum placed in $\mathbf{k}$-space according to the position of the bars of Fig. 9.3. Figures 9.7 (a) and (b) show the $p_{x y}$ and $p_{z}$ data, respectively. The imaging approach highlights the information contained in the raw spectra. Dispersion toward higher energy at $\Gamma$, as expected for a direct gap material [72], is immediately obvious for both states. 


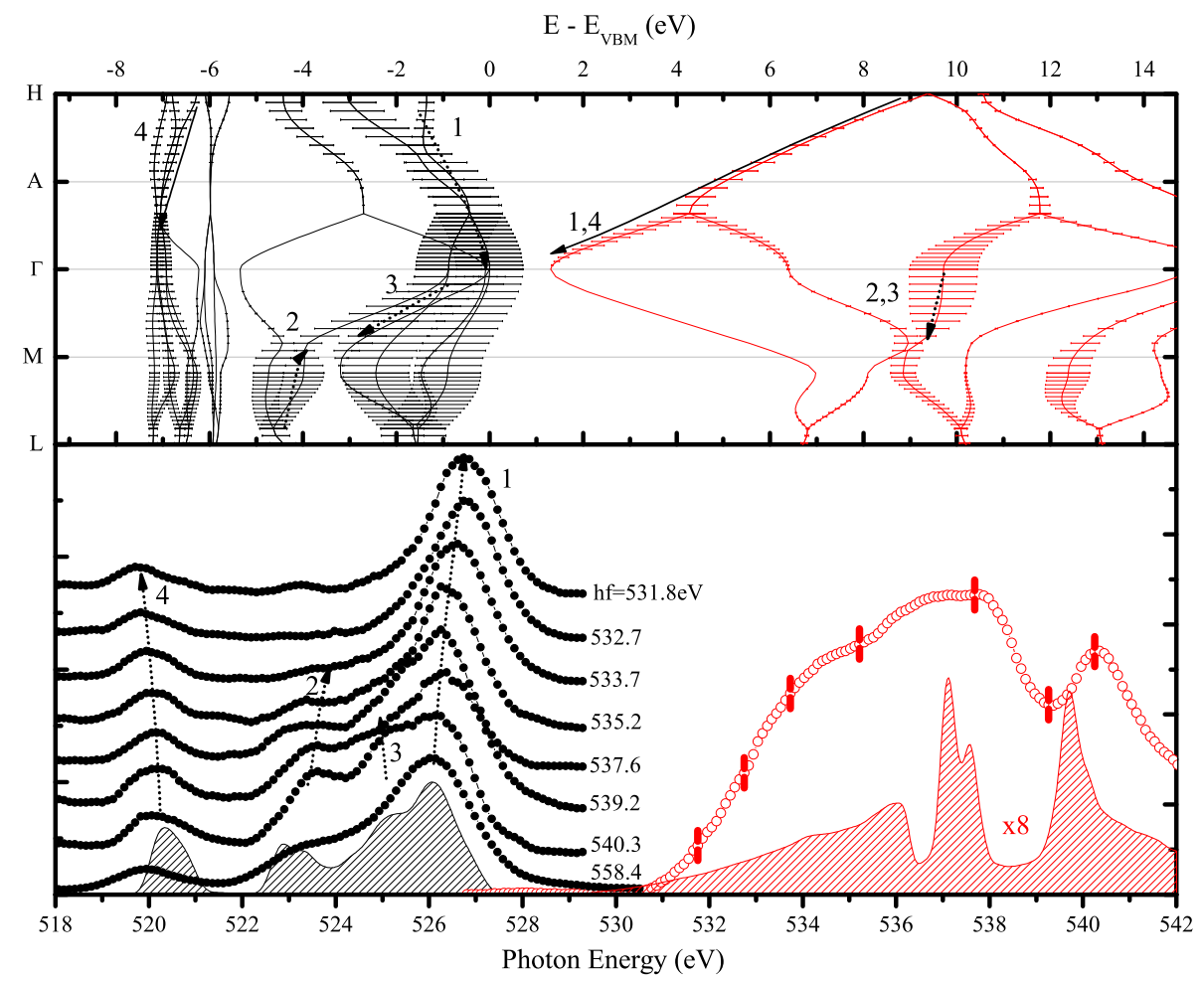

Figure 9.5: RXES (closed circles) and XAS (open circles) measured in the orientation that accentuates the $p_{x y}\left(p_{z}\right)$ contributions. The top half of the figure shows the band structure along relevant symmetry directions. The bottom half shows experimental data and also the calculated PDOS (hatched lines).

For the $p_{x y}$ states, dispersion along $\Gamma-\mathrm{A}-\mathrm{H}-\mathrm{K}$ is clearly resolved with the upper band dispersing to lower energy. The bands around $-4 \mathrm{eV}$ decrease in energy and increase in intensity along A-H-K, in line with theory. The position of the $\mathrm{Zn} 3 d$ states is apparent, just below the theoretical bands. The spectrum obtained with an excitation energy of $537.6 \mathrm{eV}$ is located near both $\mathrm{M}$ and $\mathrm{K}$, out of order with the other spectra, because of the strongly localised states in the conduction band at $10.5 \mathrm{eV}$.

The $p_{z}$ states add $\mathrm{M}-\Gamma$ and $\mathrm{K}-\Gamma$ to the parts of the Brillouin zone we can resolve. The lower energy band near $-4 \mathrm{eV}$ disperses to $-5.5 \mathrm{eV}$ approaching 


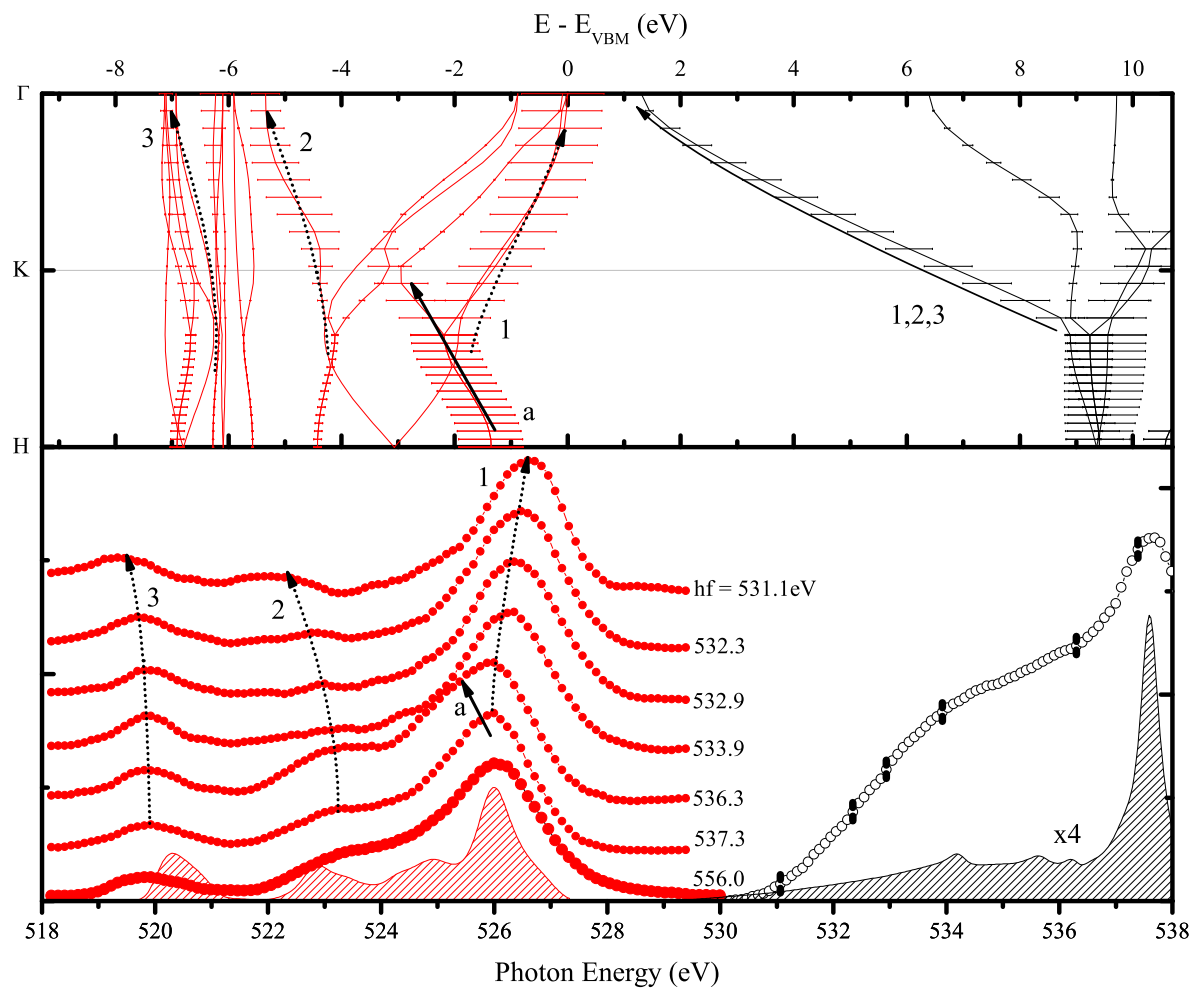

Figure 9.6: RXES (closed circles) and XAS (open circles) measured in the orientation that accentuates the $p_{z}\left(p_{x y}\right)$ contributions. The top half of the figure shows the band structure along relevant symmetry directions. The bottom half shows experimental data and also the calculated PDOS (hatched lines).

$\Gamma$, in direct contrast to the observed behaviour of the $p_{x y}$ band in this energy range and in agreement with predictions.

There has been much discussion about the correct use of intermediate and final states in RXES calculations [72, 99, 119]. We note that if the corehole correction was applied to the unoccupied states of Fig. 9.3 (a downward shift of the theory by $1.0 \mathrm{eV}$ ) the $p_{x y}$ spectrum taken with $537.6 \mathrm{eV}$ would be relocated to near $\Gamma$ and the strong intensity around $-4.5 \mathrm{eV}$ would be anomalous. This is clear evidence for the use of the final-state rule in RXES 


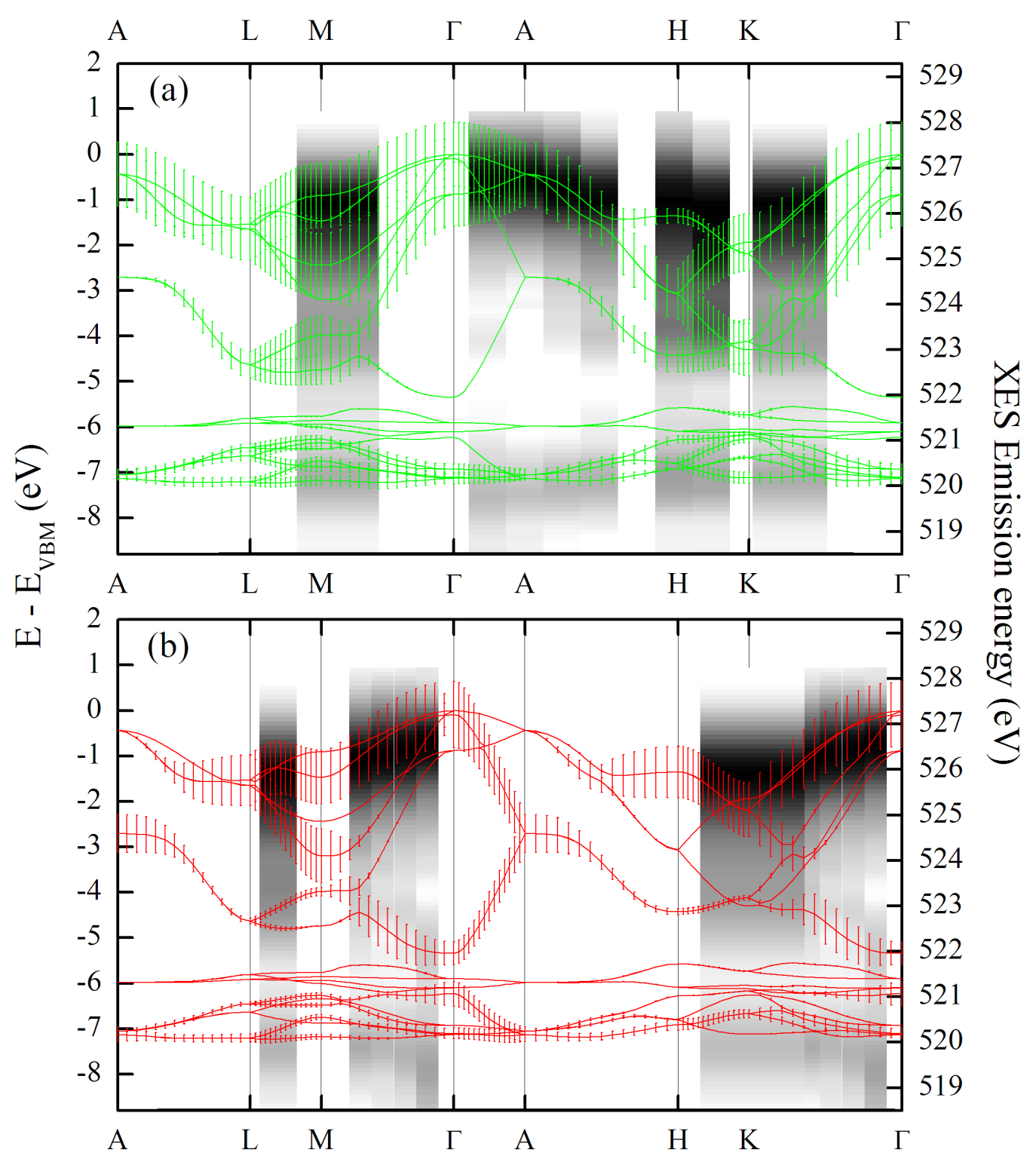

Figure 9.7: The coherent RXES overlaid with orbitally resolved band structure: (a) $\mathrm{p}_{x y}$ and (b) $\mathrm{p}_{z}$ orbitals. Intensity scales from zero (white) to one (black). 
and for the validity of the present approach which, although it is a rough approximation of the absolutely correct approach of calculating RXES spectra from the Kramers-Heisenberg equation, is much more intuitive.

\subsection{Conclusion}

In conclusion, the strong anisotropy and dispersion of wurtzite $\mathrm{ZnO}$, the k-selectivity of the RXES technique, and an $\mathrm{HDFT}+G W$ calculation were harnessed to construct a band mapping of $\mathrm{ZnO}$ across a wide range of high symmetry points. The energy of the $\mathrm{Zn} 3 d$ core level is located and evidence for the use of the final-state rule in RXES is presented. It should be possible to use further high resolution RXES experiments to tune theoretical calculations across the technologically important post transition metal oxide series. 


\section{Chapter 10}

\section{Conclusion and Outlook}

\section{$10.1 \quad$ Results}

The goal of this thesis was to advance the state of knowledge about the electronic structure of the ReNs. Significant progress was made toward that goal using a number of experimental and theoretical techniques. X-ray spectroscopy in particular played a key role.

High quality thin films of SmN, EuN, GdN, DyN, LuN and HfN were grown using TED and/or PLD. A variety of characterization techniques were employed to determine the crystalline quality of the films. RBS and NRA indicated that the films were stoichiometric. XRD and RHEED showed that most samples were polycrystalline although epitaxial GdN was grown by PLD on YSZ substrates. Capping layers that allow ex situ measurement were developed; YSZ was found to be an excellent capping layer for XXS by TFY, while Cr was proved to form a CrN interface which interferes with $\mathrm{N}$ K-edge spectroscopy.

Transport, magnetization and optical transmission measurements showed that $\mathrm{SmN}, \mathrm{GdN}$, and DyN are semiconducting ferromagnets, and that $\mathrm{SmN}$ has a near-zero moment in its ferromagnetic ground state. Temperature dependent optical transmission measurements of GdN showed a red-shift in the ferromagnetic ground state, providing direct experimental evidence for 
spin-splitting in the valence and conduction bands near the gap. With a refined $U_{d}$ parameter, used to correct for the problem that the LSDA typically underestimates the long-range Coulomb repulsion, the direct band-gap extracted from LSDA $+U$ calculations is in agreement with the ferromagnetic and room temperature optical gaps.

The tuned band structure showed excellent agreement with GdN XXS. RXES of GdN provided evidence for dispersion near the CBM and enabled some of the GdN $5 d$ bands to be located. XXS at the N K-edge of SmN, DyN, $\mathrm{LuN}$ and HfN were also measured. The SmN and DyN experimental data agreed well with calculated PDOS, while a comparison of the series showed systematic shifts in peak locations, but little evidence to allow the rare-earth $4 f$ levels to be located.

XAS at the rare-earth M-edge showed that the localized $4 f$ levels are atomic-like (in that the measured spectrum is not significantly affected by the bonding environment). Hartree-Fock calculations of the XAS suggested that the $\mathrm{LSDA}+U J_{f}$ value should be scaled to $\sim 80 \%$ of the atomic values and XAS of EuN suggested that $\mathrm{Eu}^{3+}$ is the dominant phase in EuN. HfN was shown to have a similar crystal and electronic structure to the ReNs, but a Fermi edge is evident in the XXS (HfN is metallic, unlike the ReNs).

The x-ray spectroscopy techniques used in the investigation of the ReNs lent themselves to an advanced study of ZnO. XAS, XES and RXES were performed on a high quality $\mathrm{ZnO}$ single crystal. The combined hybrid DFT calculation with $G W$ corrections was shown to be in excellent agreement with the measured XXS, accurately accounting for the strong linear dichrosim which is present due to the crystal anisotropy. The coherent part of the RXES was used to measure the valence band dispersion through a large part of the Brillouin zone. 


\subsection{Outlook}

Further progress toward determining the electronic structure of the ReNs will depend heavily on the availability of high quality samples. Therefore, in the near future a focus on improved film growth techniques is important. Essentially, a full study of the effect of the growth phase space - substrate type and temperature, nitrogen partial pressure and plasma energy, and rareearth deposition rate - on interesting film parameters - epitaxial growth and nitrogen vacancy concentration in particular - is required.

Controlled growth of epitaxial layers will allow ARPES measurements which will provide a definitive characterization of the valence band electronic structure. It will be particularly interesting to perform temperature dependent measurements to determine any differences between the band structures in the ferromagnetic and paramagnetic phases.

The tuned theoretical parameters introduced in this thesis allow for more precise predictions of ReN electronic structure. In the medium term this theoretical knowledge should be combined with advances in growth techniques to focus on band structure tuning and device construction. For example, adding $\mathrm{Hf}$ or $\mathrm{Lu}$, which have smaller ionic radii than Gd could reduce the lattice constant of GdN, resulting in a reduced band-gap and potentially even a transition from a semiconducting to half-metallic ground state.

There is a chance that the ferromagnetic transition temperature can be controlled in a similar manner. However, a much better understanding of the mechanism of the magnetism in ReNs is essential before any certainty can be attached to such a statement. Indeed this is a very interesting problem where there is presently disagreement between theoretically predicted and experimentally measured magnetic transition temperatures.

Theoretical calculations can also be improved in certain fundamental ways. The main issue is how to accurately incorporate the localized $4 f$ electrons into band structure calculations. The LSDA $+U$ codes are a step in this direction, and further tuning of the $U$ and $J$ parameters is interesting. An extension of the codes to output parameters which can be directly com- 
pared to more advanced measurement techniques, like ReN N K-edge XMCD or RXES, would simplify experimental analysis significantly and move these techniques into the realm of utility of XXS (where the ability to compare experimental data directly with numerical calculations is considered a huge strength). Eventually however, the LSDA $+U$ calculations must be superseded by $G W$ frameworks, which are fundamentally more accurate.

In the far future investigating devices based on the ReNs is clearly of interest. SmN, which has been shown to be a semiconducting ferromagnet with a near-zero fringe field, has great potential for future applications where spin polarized devices are needed but fringe fields are undesirable. For example, a scanning tunneling microscope tip capped with SmN could probe spin polarized nanoparticles without affecting them. In principle it should be possible to reduce the fringe field even further by doping either orbital or spin moment into $\mathrm{SmN}$ (depending on the character of the moment on the Sm ion), perhaps even reducing the net moment to zero.

There is an exciting future ahead of us, and a good chance that the ReNs could play a role in the technological and fundamental developments that will inevitably appear in the field of materials science. Hopefully the new results contained within this thesis will contribute to these advances. 


\section{Bibliography}

[1] R. P. Martin, Electronic Structure: Basic Theory and Practical Methods (Cambridge University Press) (2004). See page 2.

[2] S. Bandyopadhyay and M. Cahay, Introduction to Spintronics (CRC) (2008). See page 2 .

[3] I. Žutić, J. Fabian, and S. Das Sarma, Spintronics: Fundamentals and applications, Rev. Mod. Phys. 76, 323 (2004). See page 2.

[4] A. Fert, Nobel lecture: Origin, development, and future of spintronics, Rev. of Mod. Phys. 80, 1517 (2008). See page 2.

[5] Everspin Technologies, Inc., Website (2009), http://www.everspin. com. See page 2 .

[6] T. Jungwirth, J. Sinova, J. Mašek, J. Kučera, and A. H. MacDonald, Theory of ferromagnetic (III,Mn)V semiconductors, Rev. Mod. Phys. 78, 809 (2006). See page 3.

[7] S. Dhar, O. Brandt, M. Ramsteiner, V. F. Sapega, and K. H. Ploog, Colossal magnetic moment of Gd in GaN, Phys. Rev. Lett. 94, 037205 (2005). See page 3.

[8] C. Liu, F. Yun, and H. Morkoc, Ferromagnetism of ZnO and GaN: A review, J. Mat. Sci.: Mat. Elec. 16, 1573 (2005). See page 3. 
[9] M. Venkatesan, C. B. Fitzgerald, J. G. Lunney, and J. M. D. Coey, Anisotropic ferromagnetism in substituted zinc oxide, Phys. Rev. Lett. 93, 177206 (2004). See page 3.

[10] E. L. Nagaev, Physics of magnetic semiconductors (Mir Publishers) (1983). See page 3.

[11] E. L. Nagaev and M. Samokhvalov, Colossal Magnetoresistance Materials and Other Magnetic Semiconductors (Mir Publishers) (1983). See page 3 .

[12] B. J. Ruck, Spintronics potential of rare-earth nitrides and related materials, in Nanomagnetism and Spintronics, edited by F. Nasipouri and A. Nogaret (World Scientific) (2009). See pages 4, 45, 61, and 99.

[13] P. Larson, W. R. L. Lambrecht, A. Chantis, and M. van Schilfgaarde, Electronic structure of rare-earth nitrides using the $L S D A+U$ approach: Importance of allowing $4 f$ orbitals to break the cubic crystal symmetry, Phys. Rev. B 75, 045114 (2007). See pages 4, 11, 12, 26, 28, 54, 61, $62,67,71,72,81,82,85,92,102,106$, and 107.

[14] S. Nakamura, G. Fasol, and S. J. Pearton, The Blue Laser Diode: The Complete Story (Springer) (2000). See page 9.

[15] F. Hulliger, Handbook on the Physics and Chemistry of Rare Earths (North-Holland Publishing Company), chap. 33, pp. 153-236 (1979). See pages $10,11,45,50,54,85,86$, and 88 .

[16] O. Vogt and K. Mattenberger, Magnetic measurements on rare earth and and actinide monopnictides and monchalcogenides (Elsevier, Amsterdam), vol. 17, chap. 114, pp. 301 - 407 (1993). See pages 10, 11, 85 , and 88 .

[17] Busch, Kaldis, Schaufelberger-Teker, and Wachter, Synthesis, crystal growth and physical properties of rare-earth nitride phases, in Proceed- 
ings of Colloque International du C.N.R.S. (Paris-Grenoble) (1969), vol. 1, p. 359. See pages 10 and 70 .

[18] R. A. Cutler and A. W. Lawson, Synthesis and magnetic behavior of GdN, J. Appl. Phys. 46, 2739 (1975). See page 10.

[19] R. C. Brown and N. J. Clark, Composition limits and vaporization behaviour of rare earth nitrides, J. inorg. nucl. Chem. 36, 2507 (1974). See pages 10 and 11 .

[20] E. Shalaan and H. Schmitt, Structural and optical properties of sputtered gadolinium nitride films, Opt. Commun. 260, 588 (2005). See page 10 .

[21] N. Sclar, Properties of rare-earth nitrides, J. Appl. Phys. 35, 1534 (1964). See pages 11 and 86.

[22] T. R. McGuire, R. J. Gambino, S. J. Pickart, and H. A. Alperin, Magnetic structure and exchange interactions in cubic gadolinium compounds, J. Appl. Phys. 40, 1009 (1969). See page 11.

[23] P. Wachter and E. Kaldis, Magnetic interaction and carrier concentration in $G d N$ and $G d N_{1-x} O_{x}$, Solid State Com. 34, 241 (1980). See page 11 .

[24] R. Didchenko and F. P. Gortsema, Some electric and magnetic properties of rare earth monosulfides and nitrides, J. Phys. Chem. Solids 24, 863 (1963). See pages 11, 85, and 107.

[25] L. Degiorgi, W. Bacsa, and P. Wachter, Electronic structure of YbN, Phys. Rev. B 42, 530 (1990). See pages 11 and 107.

[26] N. Sclar, Energy gaps of the III- $V$ and the (rare earth)- $V$ semiconductors, J. Appl. Phys. 33, 2999 (1962). See page 11. 
[27] H. R. Child, M. K. Wilkinson, J. W. Cable, W. C. Koehler, and E. O. Wollan, Neutron diffraction investigation of the magnetic properties of compounds of rare-earth metals with group $V$ anions, Phys. Rev. 131, 922 (1963). See page 11.

[28] D. P. Schumacher and W. E. Wallace, Magnetic characteristics of gadolinium, praseodymium, and thulium nitrides, J. Appl. Phys. 36, 984 (1965). See page 11.

[29] G. Busch, Magnetic properties of rare-earth compounds, J. Appl. Phys. 38, 1386 (1967). See pages 11 and 85.

[30] D. P. Schumacher and W. E. Wallace, Magnetic characteristics of some lanthanide nitrides, Inorg. Chem. 5, 1563 (1966). See pages 11, 85, and 107 .

[31] D. X. Li, Y. Haga, H. Shida, T. Suzuki, Y. S. Kwon, and G. Kido, Magnetic properties of stoichiometric Gd monopnictides, J. Phys.: Condens. Matter 9, 10777 (1997). See pages 11 and 62.

[32] R. J. Gambino, T. R. McGuire, H. A. Alperin, and S. J. Pickart, Magnetic properties and structure of $G d N$ and $G d N_{1-x} O_{x}$, J. Appl. Phys. 41, 933 (1970). See page 11.

[33] M. Kuznietz, Effect of substitutional anions on the magnetic properties of the mononitrides of uranium and gadolinium, J. Appl. Phys. 42, 1470 (1971). See page 11.

[34] R. J. Gambino, D. E. Eastman, T. R. McGuire, V. L. Moruzzi, and W. D. Grobman, Evidence of the intrinsic metallic character of some rare-earth pnictides, J. Appl. Phys. 42, 1468 (1971). See page 11.

[35] H. Yamada, T. Fukawa, T. Muro, Y. Tanaka, S. Imada, S. Suga, D.-X. $\mathrm{Li}$, and T. Suzuki, XPS and X-BIS studies of Gd monopnictides, J. Phys. Soc. Jpn. 65, 1000 (1996). See page 11. 
[36] C. M. Aerts, P. Strange, M. Horne, W. M. Temmerman, Z. Szotek, and A. Svane, Half-metallic to insulating behavior of rare-earth nitrides, Phys. Rev. B 69, 045115 (2004). See pages 11, 12, 61, and 85.

[37] C.-G. Duan, R. F. Sabiryanov, J. Liu, W. N. Mei, P. A. Dowben, and J. R. Hardy, Strain induced half-metal to semiconductor transition in GdN, Phys. Rev. Lett. 94, 237201 (2005). See pages 11 and 61.

[38] C.-G. Duan, R. F. Sabirianov, W. N. Mei, P. A. Dowben, S. S. Jaswal, and E. Y. Tsymbal, Electronic, magnetic and transport properties of rare-earth monopnictides, J. Phys.: Condens. Matter 19, 315220 (2007). See pages 11, 45, 61, and 85 .

[39] F. Leuenberger, Elemental resolved magnetism of gadolinium nitride layers and $G d N / F e$ multilayers, Ph.D. thesis, Georg-AugustUniversität zu Göttingen (2004). See pages 12, 47, 62, and 67.

[40] F. Leuenberger, A. Parge, W. Felsch, F. Baudelet, C. Giorgetti, E. Dartyge, and F. Wilhelm, X-ray magnetic circular dichroism at the $G d L_{2,3}$ absorption edges in GdN layers: The influence of lattice expansion, Phys. Rev. B 73, 214430 (2006). See pages 12, 62, 71, and 83.

[41] F. Leuenberger, A. Parge, W. Felsch, K. Fauth, and M. Hessler, GdN thin films: Bulk and local electronic and magnetic properties, Phys. Rev. B 72, 014427 (2005). See pages 12, 32, 47, 52, 59, 61, 62, 64, 92, and 96 .

[42] C. Stampfl, W. Mannstadt, R. Asahi, and A. J. Freeman, Electronic structure and physical properties of early transition metal mononitrides: Density-functional theory LDA, GGA, and screened-exchange LDA FLAPW calculations, Phys. Rev. B 63, 155106 (2001). See pages 12 and 54 .

[43] E. Kaxiras, Atomic and Electronic Structure of Solids (Cambridge University Press) (2003). See pages 15, 16, and 17. 
[44] P. Hohenberg and W. Kohn, Inhomogeneous electron gas, Phys. Rev. 136, B864 (1964). See page 16.

[45] W. Kohn and L. J. Sham, Self-consistent equations including exchange and correlation effects, Phys. Rev. 140, A1133 (1965). See page 16.

[46] R. D. Cowan, The theory of atomic structure and spectra (University of California Press) (1981). See pages 19, 20, 21, and 25.

[47] B. T. Thole, G. van der Laan, J. C. Fuggle, G. A. Sawatzky, R. C. Karnatak, and J.-M. Esteva, $3 d$ x-ray-absorption lines and the $3 d^{9} 4 f^{n+1}$ multiplets of the lanthanides, Phys. Rev. B 32, 5107 (1985). See pages 19, 21, 81, 85, 89, 106, and 108.

[48] J. B. Goedkoop, B. T. Thole, G. van der Laan, G. A. Sawatzky, F. M. F. de Groot, and J. C. Fuggle, Calculations of magnetic x-ray dichroism in the $3 d$ absorption spectra of rare-earth compounds, Phys. Rev. B 37, 2086 (1988). See page 19.

[49] F. de Groot, Multiplet effects in x-ray spectroscopy, Coord. Chem. Rev. 249, 31 (2005). See pages 19, 20, 21, 22, and 26.

[50] G. van der Laan, Hitchhiker's guide to multiplet calculations, in Magnetism: A Synchrotron Radiation Approach (Springer Berlin) (2006). See pages 20 and 21.

[51] R. Gusmeroli and C. Dallera, Missing, Website (2009), http://www . esrf .eu/UsersAndScience/Experiments/TBS/SciSoft/OurSoftware/ MISSING. See page 26.

[52] M. Methfessel, M. van Schilfgaarde, and R. A. Casali, A full-potential LMTO method based on smooth hankel functions, in Electronic Structure and Physical Properties of Solids: The Uses of the LMTO Method, Lecture Notes in Physics 535, edited by H. Dreysse (Springer-Verlag, Berlin) (2000). See page 26. 
[53] P. Larson and W. R. L. Lambrecht, Electronic structure of Gd pnictides calculated within the $L S D A+U$ approach, Phys. Rev. B 74, 085108 (2006). See pages 26 and 62.

[54] W. R. L. Lambrecht, Electronic structure and optical spectra of the semimetal ScAs and of the indirect-band-gap semiconductors ScN and GdN, Phys. Rev. B 62, 13538 (2000). See pages 27 and 68.

[55] V. I. Anisimov, J. Zaanen, and O. K. Andersen, Band theory and Mott insulators: Hubbard U instead of Stoner I, Phys. Rev. B 44, 943 (1991). See page 27.

[56] A. I. Liechtenstein, V. I. Anisimov, and J. Zaanen, Density-functional theory and strong interactions: Orbital ordering in Mott-Hubbard insulators, Phys. Rev. B 52, R5467 (1995). See pages 27 and 28.

[57] N. F. Mott and E. A. Davis, Electronic processes in non-crystalline materials (Oxford) (1979). See page 32.

[58] B. J. Ruck, Vortex dynamics and instabilities in $T a_{x} G e_{1-x} / G e$ Multilayers, Ph.D. thesis, Victoria University of Wellington (1998). See page 34.

[59] N. W. Ashcroft and N. D. Mermin, Solid state physics (Saunders College) (1976). See pages 35 and 52.

[60] A. M. Stewart, Paramagnetic susceptibilities of metallic samarium compounds, Phys. Rev. B 6, 1985 (1972). See page 35.

[61] C. Mitra and W. R. L. Lambrecht, Calculated interband optical transition spectra of GdN, Phys. Rev. B 78, 195203 (2008). See page 36.

[62] R. Denecke, P. Vterlein, M. Bssler, N. Wassdahl, S. Butorin, A. Nilsson, J. E. Rubensson, J. Nordgren, N. Mrtensson, and R. Nyholm, Beamline I511 at MAX II, capabilities and performance, J. Elec. Spec. Rel. Phenom. 101-103, 971 (1999). See page 37. 
[63] J. Stöhr, NEXAFS Spectroscopy, Springer Series in Surface Sciences (Springer), 2nd ed. (2003). See pages 38, 39, and 40.

[64] B. Lengeler, X-ray absorption and reflection in materials sciences, Adv. Solid State Phys. 29, 53 (1989). See page 38.

[65] E. J. Nordgren, Soft x-ray emission spectroscopy in the nineties, J. Elec. Spec. Rel. Phenom. 78, 25 (1996). See pages 38 and 41.

[66] A. C. Thompson and D. Vaughan, X-ray data booklet, online (2001). See pages 38,39 , and 78 .

[67] J. E. Downes, Soft X-ray Spectroscopic Studies of the Electronic Structure of Organic Molecular Superconductors and Semiconductors, Ph.D. thesis, Boston University (2003). See page 40.

[68] U. von Barth and G. Grossmann, Dynamical effects in x-ray spectra and the final-state rule, Phys. Rev. B 25, 5150 (1982). See pages 41 and 116.

[69] G. D. Mahan, Final-state potential in x-ray spectra, Phys. Rev. B 21, 1421 (1980). See page 41.

[70] J. Luitz, M. Maier, C. Hébert, P. Schattschneider, P. Blaha, K. Schwarz, and B. Jouffrey, Partial core hole screening in the Cu $L_{3}$ edge, Eur. Phys. J. B 21, 363 (2001). See page 41.

[71] J. Nordgren and N. Wassdahl, Soft x-ray fluorescence spectroscopy using tunable synchrotron radiation, J. Elec. Spec. Rel. Phenom. 72, 273 (1995). See page 41.

[72] S. Eisebitt and W. Eberhardt, Band structure information and resonant inelastic soft x-ray scattering in broad band solids, J. Elec. Spec. Rel. Phenom. 110, 335 (2000). See pages 42, 78, 79, 119, 120, and 122. 
[73] J. A. Carlisle, S. R. Blankenship, R. N. Smith, E. L. Shirley, L. J. Terminello, J. J. Jia, T. A. Callcott, and D. L. Ederer, Soft-x-ray fluorescence studies of solids, J. Elec. Spec. Rel. Phenom. 101-103, 839 (1999). See page 42.

[74] W. Schattke and M. A. Van Hove (editors), Solid-state photoemission and related methods: theory and experiment (Wiley) (2003). See pages 44 and 111.

[75] J. W. Gerlach, J. Mennig, and B. Rauschenbach, Epitaxial gadolinium nitride thin films, Appl. Phys. Lett. 90, 061919 (2007). See pages 47, 49 , and 62 .

[76] M. A. Scarpulla, C. S. Gallinat, S. Mack, J. S. Speck, and A. C. Gossard, GdN (111) heteroepitaxy on GaN (0001) by $\mathrm{N}_{2}$ plasma and $\mathrm{NH}_{3}$ molecular beam epitaxy, Journal of Crystal Growth in press (2009). See page 47 .

[77] S. E. Granville, Structural, electronic and magnetic properties of GaMnN, MnN and rare-earth nitride thin films, Ph.D. thesis, Victoria University of Wellington (2007). See page 47.

[78] S. E. Granville, B. J. Ruck, F. Budde, A. Koo, D. J. Pringle, F. Kuchler, A. R. H. Preston, D. H. Housden, N. Lund, A. Bittar, G. V. M. Williams, and H. J. Trodahl, Semiconducting ground state of GdN thin films, Phys. Rev. B 73, 235335 (2006). See pages 47, 52, 64, and 67.

[79] R. F. Davis and M. S. Shur, GaN-based Materials and Devices: Growth, Fabrication, Characterization \& 8 Performance (World Scientific Publishing Company) (2004). See pages 48 and 49.

[80] H. V. Suu, G. Petõ, G. Mezey, F. Pászti, E. Kótai, M. Fried, A. Manuaba, E. Zsoldos, and J. Gyulai, Formation of $\mathrm{GdSi}_{2}$ under UHV evaporation and in situ annealing, Appl. Phys. Lett. 48, 437 (1986). See page 49 . 
[81] A. Schmehl, V. Vaithyanathan, A. Herrnberger, S. Thiel, C. Richter, M. Liberati, T. Heeg, M. Rockerath, L. F. Kourkoutis, S. Muhlbauer, P. Boni, D. A. Muller, Y. Barash, J. Schubert, Y. Idzerda, J. Mannhart, and D. G. Schlom, Epitaxial integration of the highly spin-polarized ferromagnetic semiconductor EuO with silicon and GaN, Nat. Mater. 6, 882 (2007). See pages 49 and 68.

[82] B. Ludbrook, I. L. Farell, M. Kuebel, B. J. Ruck, A. R. H. Preston, H. J. Trodahl, L. Ranno, R. J. Reeves, and S. M. Durbin, Growth and properties of epitaxial GdN, J. Appl. Phys 106, 063910 (2009). See pages 49, 52, 57, 64, 67, and 76 .

[83] T. Suehiro, N. Hirosaki, Y. Yamamoto, T. Nishimura, and M. Mitomo, Preparation of lutetium nitride by direct nitridation, J. Mater. Res. 19, 959 (2004). See page 54.

[84] I. L. Farrell, R. J. Reeves, S. M. Durbin, A. R. H. Preston, and B. J. Ruck, Electrical and optical properties of hafnium nitride thin films, Appl. Phys. Lett. submitted (2009). See page 54.

[85] L. V. Azaroff and M. J. Buerger, The Powder Method (McGraw-Hill) (1958). See page 55 .

[86] P. G. Steeneken, New light on EuO thin films: Preparation, transport, magnetism and spectroscopy of a ferromagnetic semiconductor, Ph.D. thesis, Rijksuniversiteit Groningen (2002). See pages 61 and 64.

[87] V. N. Antonov, B. N. Harmon, A. N. Yaresko, and A. P. Shpak, Xray magnetic circular dichroism in GdN: First-principles calculations, Phys. Rev. B 75, 184422 (2007). See page 61.

[88] A. N. Chantis, M. van Schilfgaarde, and T. Kotani, Quasiparticle selfconsistent $G W$ method applied to localized $4 f$ electron systems, Phys. Rev. B 76, 165126 (2007). See page 62. 
[89] C.-G. Duan, R. F. Sabiryanov, W. N. Mei, P. A. Dowben, S. S. Jaswal, and E. Y. Tsymbal, Magnetic ordering in Gd monopnictides: Indirect exchange versus superexchange interaction, Appl. Phys. Lett. 88, 182505 (2006). See pages 62 and 72.

[90] P. Y. Yu and M. Cardona, Fundamentals of semiconductors (Springer) (1999). See page 64.

[91] C.-G. Duan, R. F. Sabiryanov, J. Liu, W. N. Mei, P. A. Dowben, and J. R. Hardy, Theoretical study of the magnetic ordering in rareearth compounds with face-centered-cubic structure, J. Appl. Phys. 97, 10 A915 (2005). See page 67.

[92] C. Mitra and W. R. L. Lambrecht, Magnetic exchange interactions in the gadolinium pnictides from first principles, Phys. Rev. B 78, 134421 (2008). See page 67.

[93] G. van der Laan, E. Arenholz, A. Schmehl, and D. G. Schlom, Weak anisotropic x-ray magnetic linear dichroism at the Eu $M_{4,5}$ edges of ferromagnetic EuO(001): Evidence for $4 f$-state contributions, Phys. Rev. Lett. 100, 067403 (2008). See page 67.

[94] H. J. Trodahl, A. R. H. Preston, J. Zhong, B. J. Ruck, N. M. Strickland, C. Mitra, and W. R. L. Lambrecht, Ferromagnetic redshift of the optical gap in GdN, Phys. Rev. B 76, 085211 (2007). See pages 69, 72, and 73.

[95] A. R. H. Preston, S. Granville, D. H. Housden, B. Ludbrook, B. J. Ruck, H. J. Trodahl, A. Bittar, G. V. M. Williams, J. E. Downes, A. DeMasi, Y. Zhang, K. E. Smith, and W. R. L. Lambrecht, Comparison between experiment and calculated band structures for DyN and SmN, Phys. Rev. B 76, 245120 (2007). See pages 74, 76, and 89.

[96] A. R. H. Preston, B. J. Ruck, L. F. J. Piper, A. DeMasi, K. E. Smith, A. Schleife, F. Fuchs, F. Bechstedt, J. Chai, and S. M. Durbin, Band 
structure of ZnO from resonant x-ray emission spectroscopy, Phys. Rev. B 78, 155114 (2008). See pages 75 and 79 .

[97] V. N. Strocov, T. Schmitt, J.-E. Rubensson, P. Blaha, T. Paskova, and P. O. Nilsson, Momentum selectivity and anisotropy effects in the nitrogen K-edge resonant inelastic x-ray scattering from GaN, Phys. Rev. B 72, 085221 (2005). See pages 76, 79, and 114.

[98] A. Kotani and S. Shin, Resonant inelastic x-ray scattering spectra for electrons in solids, Rev. Mod. Phys. 73, 203 (2001). See page 79.

[99] D. Eich, O. Fuchs, U. Groh, L. Weinhardt, R. Fink, E. Umbach, C. Heske, A. Fleszar, W. Hanke, E. K. U. Gross, C. Bostedt, T. v. Buuren, N. Franco, L. J. Terminello, M. Keim, G. Reuscher, H. Lugauer, and A. Waag, Resonant inelastic soft x-ray scattering of Be chalcogenides, Phys. Rev. B 73, 115212 (2006). See pages 79 and 122.

[100] L. Weinhardt, O. Fuchs, E. Umbach, C. Heske, A. Fleszar, W. Hanke, and J. D. Denlinger, Resonant inelastic soft x-ray scattering, $x$-ray absorption spectroscopy, and density functional theory calculations of the electronic bulk band structure of CdS, Phys. Rev. B 75, 165207 (2007). See page 79 .

[101] C. Meyer, B. J. Ruck, J. Zhong, S. Granville, A. R. H. Preston, G. V. M. Williams, and H. J. Trodahl, Near-zero-moment ferromagnetism in the semiconductor SmN, Phys. Rev. B 78, 174406 (2008). See page 89.

[102] B. J. Ruck, A. Koo, U. D. Lanke, F. Budde, S. Granville, H. J. Trodahl, A. Bittar, J. B. Metson, V. J. Kennedy, and A. Markwitz, Quantitative study of molecular $\mathrm{N}_{2}$ trapped in disordered GaN:O films, Phys. Rev. B 70, 235202 (2004). See page 96.

[103] A. P. Ramirez, Applied physics: Oxide electronics emerge, Science 315, 1377 (2007). See page 111. 
[104] U. Özgür, Y. I. Alivov, C. Liu, A. Teke, M. A. Reshchikov, S. Doğan, V. Avrutin, S.-J. Cho, and H. Morkoç, A comprehensive review of ZnO materials and devices, J. Appl. Phys. 98, 041301 (2005). See pages 112 and 116.

[105] W. Göpel, J. Pollmann, I. Ivanov, and B. Reihl, Angle-resolved photoemission from polar and nonpolar zinc oxide surfaces, Phys. Rev. B 26, 3144 (1982). See page 112.

[106] K. Ozawa, K. Sawada, Y. Shirotori, and K. Edamoto, Angle-resolved photoemission study of the valence band structure of $Z_{n} O(10 \overline{10})$, J. Phys. Cond. Mat. 17, 1271 (2005). See page 112.

[107] R. A. Powell, W. E. Spicer, and J. C. McMenamin, Location of the Zn $3 d$ states in ZnO, Phys. Rev. Lett. 27, 97 (1971). See page 112.

[108] C. J. Vesely, R. L. Hengehold, and D. W. Langer, UV photoemission measurements of the upper d levels in the IIB-VIA compounds, Phys. Rev. B 5, 2296 (1972). See page 112.

[109] L. Ley, R. A. Pollak, F. R. McFeely, S. P. Kowalczyk, and D. A. Shirley, Total valence-band densities of states of III-V and II-VI compounds from x-ray photoemission spectroscopy, Phys. Rev. B 9, 600 (1974). See page 112 .

[110] C. McGuinness, C. B. Stagarescu, P. J. Ryan, J. E. Downes, D. Fu, K. E. Smith, and R. G. Egdell, Influence of shallow core-level hybridization on the electronic structure of post-transition-metal oxides studied using soft x-ray emission and absorption, Phys. Rev. B 68, 165104 (2003). See page 112.

[111] C. L. Dong, C. Persson, L. Vayssieres, A. Augustsson, T. Schmitt, M. Mattesini, R. Ahuja, C. L. Chang, and J.-H. Guo, Electronic structure of nanostructured ZnO from x-ray absorption and emission spec- 
troscopy and the local density approximation, Phys. Rev. B 70, 195325 (2004). See page 112.

[112] J.-H. Guo, L. Vayssieres, C. Persson, R. Ahuja, B. Johansson, and J. Nordgren, Polarization-dependent soft-x-ray absorption of a highly oriented ZnO microrod-array, J. Phys. Cond. Mat. 17, 235 (2005). See pages 112 and 113.

[113] W. C. T. Lee, M. Henseler, P. Miller, C. H. Swartz, T. H. Myers, R. J. Reeves, and S. M. Durbin, Effect of annealing on the morphology and optoelectrical characteristics of $\mathrm{ZnO}$ thin films grown by plasma-assisted molecular beam epitaxy, J. Elec. Mat 35, 1316 (2006). See page 113.

[114] J. Kennedy, A. Markwitz, H. J. Trodahl, B. J. Ruck, S. M. Durbin, and W. Gao, Ion beam analysis of amorphous and nanocrystalline group III$V$ nitride and $\mathrm{ZnO}$ thin films, J. Elec. Mat 36, 472 (2007). See page 113.

[115] J. Heyd, G. E. Scuseria, and M. Ernzerhof, Hybrid functionals based on a screened coulomb potential, Journal Chem. Phys. 118, 8207 (2003). See page 114 .

[116] L. Hedin, New method for calculating the one-particle Green's function with application to the electron-gas problem, Phys. Rev. 139, A796 (1965). See page 114.

[117] M. Shishkin and G. Kresse, Implementation and performance of the frequency-dependent gw method within the PAW framework, Phys. Rev. B 74, 035101 (2006). See page 114.

[118] F. Fuchs, J. Furthmüller, F. Bechstedt, M. Shishkin, and G. Kresse, Quasiparticle band structure based on a generalized Kohn-Sham scheme, Phys. Rev. B 76, 115109 (2007). See pages 114 and 116. 
[119] J. A. Carlisle, E. L. Shirley, L. J. Terminello, J. J. Jia, T. A. Callcott, D. L. Ederer, R. C. C. Perera, and F. J. Himpsel, Band-structure and core-hole effects in resonant inelastic soft-x-ray scattering: Experiment and theory, Phys. Rev. B 59, 7433 (1999). See page 122. 